\title{
CALIBRATION OF TIME DOMAIN NETWORK ANALYZERS
}

\author{
by \\ Wansheng Su \\ Dissertation submitted to the Faculty of the \\ Virginia Polytechnic Institute and State University \\ in partial fulfillment of the requirements for the degree of \\ DOCTOR OF PHILOSOPHY \\ in
}

Electrical Engineering

APPROVED:
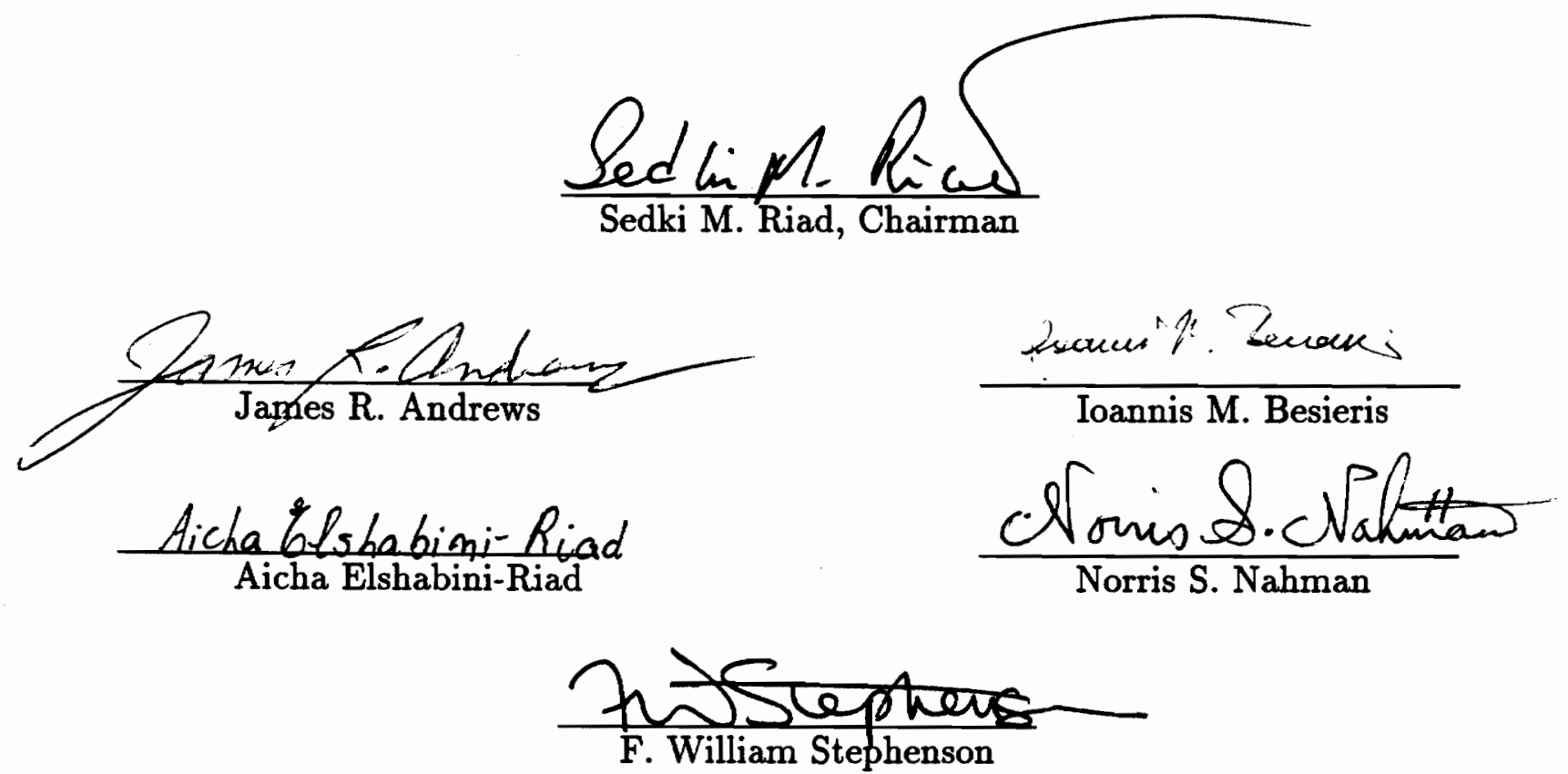

December, 1992

Blacksburg, Virginia 
C. 2

$$
\begin{aligned}
& L D \\
& 5655 \\
& 1856 \\
& 1992 \\
& 58 \\
& C .2
\end{aligned}
$$




\title{
CALIBRATION OF TIME DOMAIN NETWORK ANALYZERS
}

\author{
by \\ Wansheng Su \\ Committee Chairman: Sedki M. Riad \\ Electrical Engineering
}

(ABSTRACT)

A calibration technique for time domain reflectometry and transmission (TDR and TDT) measurement system as applied to network analysis is presented. The calibration corrects for the errors caused by the response of the measurement system. A complete physically-based model has been established for the system. A set of calculable standards has been developed to satisfy the time domain requirements for calibration. The calibration technique was applied to determining the model parameters of a commercial TDR and TDT system. The errors of modeling and de-embedding are analyzed. The calibration enhanced the system bandwidth from $8 \mathrm{GHz}$ to about $20 \mathrm{GHz}$. Experimental verification is given to demonstrate the validity and accuracy of the calibration technique. 
"When you can measure what you are speaking about, and express it in numbers, you know something about it."

Lord Kelvin 


\section{ACKNOWLEDGMENT}

I would like to thank my advisor Dr. Sedki M. Riad, for his continued encouragement, guidance and support during my entire graduate studies.

Appreciation is also extended to the members of my advisory committee, Drs. James R. Andrews, Ioannis M. Besieris, Aicha Elshabini-Riad, Norris S. Nahman, and F. William Stephenson for their guidance. All of their recommendations enhanced the quality of this study and provided me with many new insights.

I would like to acknowledge Du Pont Electronics, the Du Pont company for supporting the research pertinent to this dissertation. Also, acknowledge is due to Picosecond Pulse Labs for their equipment donation and support.

A special note of thanks is extended to my family for their care and support in all these years. 


\section{TABLE OF CONTENTS}

Chapter Title

Introduction............................................................................... 1

1.1 The Time Domain Network Analyzer (TDNA) System ............ 1

1.1.1 The Pulse Generator .................................................... 2

1.1.2 The Oscilloscope ....................................................... 4

1.1.3 The Computer ......................................................... 5

1.2 History of TDNA System and Calibration .............................. 6

1.2.1 History of TDNA System ........................................... 6

1.2.2 History of TDNA Calibration.................................... 9

1.3 Dissertation Objectives and Organization.............................. 11

1.3.1 Dissertation Objectives .............................................. 11

1.3.2 Dissertation Organization...................................... 12

$2 \quad$ Modeling the Time Domain Network Analyzers .................................. 14

2.1 Introduction .................................................................... 14

2.2 TDNA Specifications and Operation Principle........................ 16

2.2.1 System Specifications ................................................ 16

2.2.2 TDNA Principles of Operation ................................... 17

2.3 System S-Parameter Model ................................................. 18

2.4 Models for Alternate TDNA System Configurations ................. 19

2.5 Evaluation of Model Parameters ............................................ 20

$3 \quad$ Time Domain Calibration Standards ............................................... 34

3.1 Introduction ...................................................................... 34

3.2 Optimum Standard Set for TDNA Calibration........................ 36

3.3 Coaxial Transmission Line As a Calculable Standard ................ 37

3.4 Discontinuity Capacitance of Coaxial Discontinuity .................. 40

3.5 Coaxial Short and Open Circuit Standards.............................. 41

3.5.1 Coaxial Short Circuit Standard .................................... 41

3.5.2 Coaxial Open Circuit Standard ...................................... 42

3.6 Error Sources .................................................................... 43

3.6.1 Environmental Conditions........................................... 43

3.6.2 Mechanical Tolerances ..................................... . . 43 
$4 \quad$ Experimental Data Processing............................................................ 51

4.1 Introduction ................................................................. 51

$4.2 \quad$ Signal Averaging ............................................................ 52

$4.3 \quad$ Fourier Transform............................................................ 54

$4.4 \quad$ Fast Fourier Transform (FFT) ............................................ 55

$4.5 \quad$ Zero Padding ....................................................................... 58

4.6 Waveform Truncation ........................................................... 59

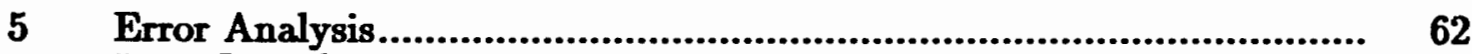

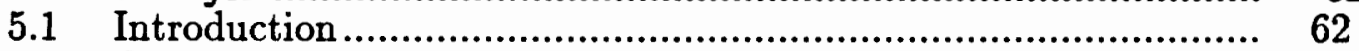

5.2 Quantization Error .............................................................. 63

$5.3 \quad$ TDR Modeling Errors ............................................................... 65

$5.4 \quad$ TDR De-Embedding Errors..................................................... 69

5.5 TDT Modeling Errors ......................................................... 71

5.6 TDT De-Embedding Errors ................................................... 73

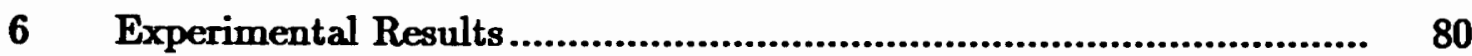

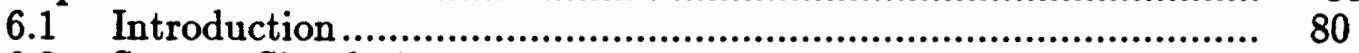

6.2 System Simulations............................................................ 80

6.2.1 Software Program Verification …………......................... 80

6.2.2 Corrections for Waveform Time Drift Errors.................... 81

6.3 Experimental Considerations............................................... 82

6.4 Modeling TDNA System ...................................................... 83

6.4.1 Acquisition of the Response Waveforms …..................... 83

6.4.2 Evaluation of Model Parameters .................................... 84

6.5 De-Embedding ................................................................... 85

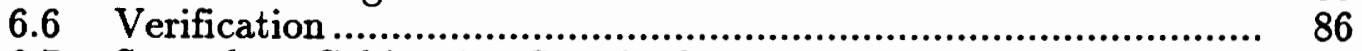

6.7 Secondary Calibration Standards....................................... 87

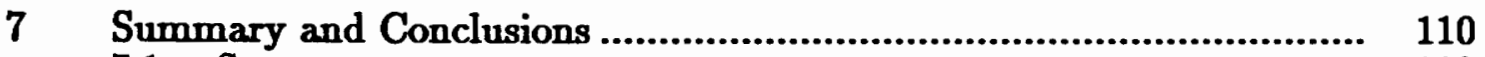

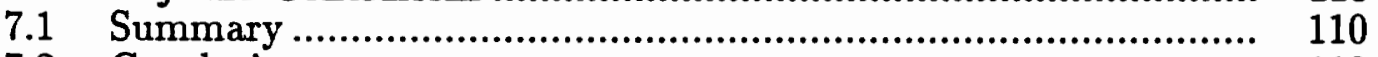

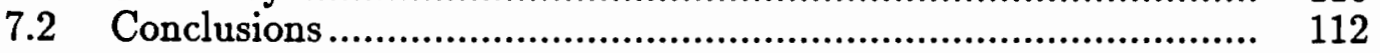




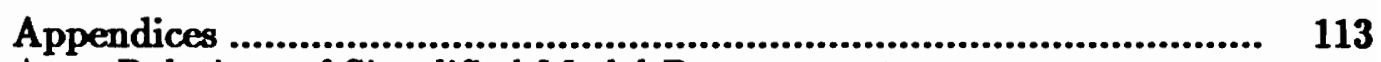

A Relations of Simplified Model Parameters to

Original Model Parameters ...................................................... 113

B Analysis of the Coaxial Open Circuit Discontinuity..................... 114

B.1 Find Mode Functions ........................................................ 114

B.2 Constructing the Field Expressions ..................................... 117

B.3 Constructing the Variational Expressions ........................... 119

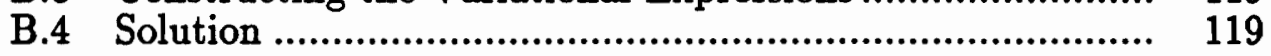

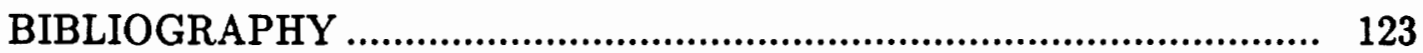

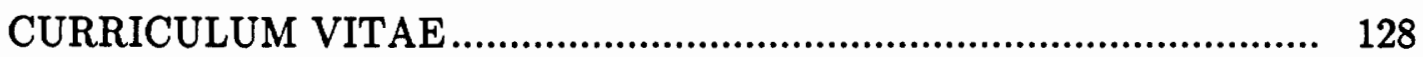




\section{LIST OF ILLUSTRATIONS}

Figure Title $\quad$ Page

1.1.1 A block diagram of TDNA system ........................................... 13

2.2.1 HP 54120 series TDNA configuration block diagram ....................... 26

2.2.2 Conceptual schematic of the TDNA system ................................... 27

2.3.1 $S$-parameter model for the TDNA system ...................................... 28

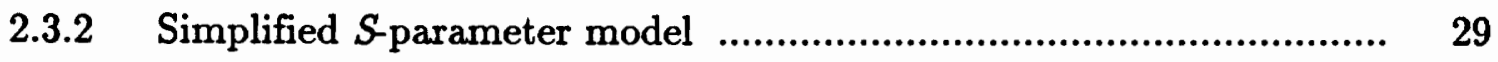

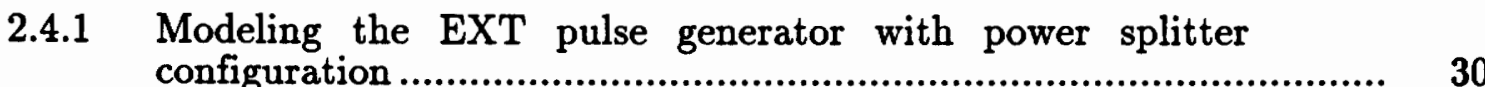

2.4.2 Modeling the feed through sampler configuration ............................ 31

2.5.1 The TDR model evaluation setup.................................................. 32

2.5.2 The TDT model evaluation setup................................................... 33

3.1.1 Offset short circuit standard (a) and its equivalent circuit (b) ........ 46

3.1.2 Offset open circuit standard (a) and its equivalent circuit (b) ........ 47

3.2.1 Variation of $\left(1-S_{11} \Gamma\right)$ with frequency ......................................... 48

3.3.1 Coaxial transmission line and its equivalent circuit ......................... 49

3.6.1 The sensitivity of the reflection coefficient $\Gamma$ versus dimension of inner conductor for a $3.5 \mathrm{~mm}$ coaxial air filled

5.2.1 ADC input voltage .................................................................... 77

5.2.2 ADC transfer characteristics ..................................................... 78

5.2.3 Quantization error versus input ................................................. 79 
6.2.1 Simulated TDR waveforms ….................................................... 89

6.2.2 Simulated TDT waveform ...................................................... 90

6.2.3 Results of the verification simulation: $S_{g r}$ amplitude versus frequency ................................................................................. 91

6.2.4 Results of the verification simulation: $S_{g 1} S_{1 r}$ amplitude versus frequency

6.2.5 Results of the verification simulation: $S_{g 1} S_{1 r}$ phase versus frequency

6.2.6 Results of the verification simulation: $S_{11}$ amplitude versus frequency

6.2.7 Results of the verification simulation: $S_{g 1} S_{2 \mathrm{t}}$ amplitude versus frequency

6.2.8 Results of the verification simulation: $S_{g 1} S_{2 t}$ phase versus frequency

6.2.9 Results of the verification simulation: $S_{z 2}$ amplitude versus frequency

6.2.10 Results of the verification simulation: $S_{11}$ amplitude versus frequency (shifting the open circuit TDR waveform 1 point forward and the short circuit TDR waveform 1 point backward)

6.2.11 Results of the verification simulation: $S_{11}$ amplitude versus frequency (same as Fig. 6.2.10 except the scale of the frequency axis)

6.2.12 Results of the verification simulation: $S_{g l} S_{1 r}$ amplitude versus frequency (shifting the open circuit TDR waveform 1 point forward and the short circuit TDR waveform 1 point backward)

6.2.13 Results of the verification simulation: $S_{g 1} S_{1 \mathrm{r}}$ amplitude versus frequency (same as Fig. 6.2.10 except the scale of the frequency axis)

6.2.14 Results of the verification simulation: $S_{g !} S_{1 r}$ amplitude versus frequency (shifting the open circuit TDR waveform 0.01 point forward and the short circuit TDR waveform 0.01 point backward) 
6.2.15 Results of the verification simulation: $S_{t 1}$ amplitude versus frequency (shifting the open circuit TDR waveform 0.01 point forward and the short circuit TDR waveform 0.01 point backward)

6.4.1 Acquired TDR response of the short circuit standard .................... 104

6.4.2 Acquired TDR response of the open circuit standard ...................... 105

6.4.3 Acquired TDR response of the $50 \Omega$ coaxial air line ...................... 106

6.4.4 Acquired TDR response of the through connection ......................... 107

6.4.5 Acquired TDT response of the through connection ......................... 108

6.5.1 Acquired and de-embedded time domain TDR response for the short circuit standard

6.5.2 Acquired TDR response of the $90 \Omega$ coaxial air line

6.5.3 De-embedded $S_{11}$ amplitude response of the $30 \Omega$ coaxial air line.

6.5.4 De-embedded $S_{11}$ phase response of the $30 \Omega$ air line ...................... 112

6.5.5 De-embedded step response of the $30 \Omega$ coaxial air line ................... 113

6.5.6 Acquired TDT response of the $10 \mathrm{~dB}$ attenuator ............................ 114

6.5.7 $S_{21}$ amplitude responses of the $10 \mathrm{~dB}$ attenuator measured by time domain and frequency domain systems.

6.5.8 De-embedded step response of the $10 \mathrm{~dB}$ attenuator

6.6.1 Comparison among the three sets of $S_{11}$ amplitude responses obtained by three different approaches for the $30 \Omega$ coaxial line

6.6.2 Comparison among the three sets of $S_{11}$ amplitude responses obtained with no calibration, simple short calibration and the complete calibration

6.6.3 The normalized signal spectrum amplitude and background noise spectrum amplitude of the system

6.6.4 Calculated error bounds for the $S_{11}$ amplitude response of the $30 \Omega$ coaxial line. Curve fitting in the Axum ${ }^{\circledR}$ software is used to obtain smooth error bound curves.... 


\section{LIST OF TABLES}

Table Title $\quad \underline{\text { Page }}$

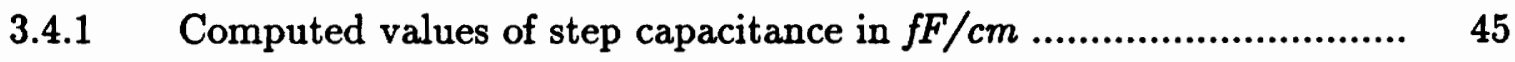




\section{CHAPTER 1}

\section{INTRODUCTION}

Experimental science depends on instrumental equipment that measures and records values of observable quantities. However, the recorded information is never exactly equal to the physical signal under test. Obtaining precise measurement data, as close to the real signal as possible, and having the knowledge to estimate the measurement uncertainty are very critical to the significance of these measurements. This dissertation presents an innovative technique for improving the accuracy of time domain network analyzers (TDNA) through calibration techniques. The first section of this chapter introduces a typical TDNA system, its configuration, and its applications. The second section reviews the history of the posed problem and presents a summary of research efforts in that regard. The third section provides an outline of the organization of the dissertation.

\subsection{THE TIME DOMAIN NETWORK ANALYZER (TDNA) SYSTEM}

A TDNA system [1] consists of three main blocks, see Fig. 1.1.1. The first block is a pulse generator which acts as a signal generator providing the required exciting 
signal. The second block is an oscilloscope, or a time domain detector, with a broad bandwidth to cover the frequency range of interest. The third block is a waveform acquisition and signal processing unit (typically a digital computer). In the following, these main sections of the TDNA are discussed in some detail. In this system, the generated pulse is launched down a reference transmission line to excite the device under test, DUT. The DUT's reflection and transmission signals can then be detected using the wideband oscilloscope. The reflection signal is known as the time domain reflectometry (TDR) signal while the transmission component is referred to as the time domain transmission (TDT) signal. Both the TDR and TDT signals contain information about the DUT. When acquired and analyzed, using the waveform acquisition and signal processing, electrical parameters of the DUT can be evaluated.

\subsubsection{The Pulse Generator}

The basic function of the pulse generator in the TDNA system is to provide the excitation or exciting signal. For a network analyzer, the requirement for the source is to provide sufficient spectrum amplitude in the frequency range of interest. It is desirable that the spectrum energy of the test pulse is concentrated with the frequency band of interest. In order to effectively use the spectrum, the ideal waveform for this kind of application should possesses maximum spectrum amplitude within the required bandwidth. This requirement can be interpreted into certain pulse shape. As known, a pulse with sinc function shape ( $\left.\frac{\sin \left[2 \pi f_{0} t\right]}{2 \pi f_{0} t}\right)$ has uniform spectrum within the bandwidth $f \leq f_{0}$ and zero outside this bandwidth. This situation would provide an ideal pulse shape for the time domain application. 
In practice, however, it is very difficult (if not impossible) to generate such a pulse shape, especially in the picosecond range. The most common commercially available pulse shape of a pulse generator is the steplike transition pulse. An ideal step pulse $u(t)$ has considerable low-frequency spectrum amplitude and a $1 / f$ frequency decline. Differentiating the steplike pulse enables one to obtain an impulse signal. An impulse signal has a relatively flatter spectrum, but its spectrum amplitude at low frequencies is less than that of the corresponding steplike waveform. One serious problem with differentiation is that it reduces the signal to noise ratio $(S / N)$ of the available pulse.

For the picosecond range, many kinds of pulse generators are available possessing different pulse parameter specifications [2]. The first class of pulse generator is the Mercury switch pulse generator. For this pulse generator, the transition duration is around hundreds of picoseconds with amplitudes reaching up into the kilovolt region. The repetition rate is relatively low, of the order of hundreds of Hertz. This generator is very useful for applications involving devices with high attenuation. Another class of pulse generator is the tunnel diode pulse generator. This generator produces a steplike output of about $250 \mathrm{mV}$ in amplitude with $10 \%$ $90 \%$ transition duration as fast as 20 ps. Repetition rates of about one hundred kilohertz can be reasonably realized. Step recovery diodes (SRD) can be used to generate higher voltage pulses with down to about $35 \mathrm{ps}$ transition duration. The fastest available electronic pulse generators use Josephson junction devices. The transition duration can reach $5 p s$ or less with amplitudes of the order of a few millivolts. 
It is important to realize that the selection of the pulse generator is not based solely on its transition duration and output level. Pulse shape, flatness of its base and top states, jitter and noise content and repetition rate play vital roles in the selection decision. If one measures a relatively low band device using a relatively fast pulse generator, the signal's fast variations (fine structure) may cause aliasing errors due to improper waveform sampling. In such a case, a low pass filter is needed to eliminate the unnecessary spectral components.

\subsubsection{The Oscilloscope}

In a TDNA system, the detector is typically an oscilloscope. For such an oscilloscope, the basic requirements are: wideband performance (fast transient response), low background noise level and linearity of both the time channel and signal channel. In general, oscilloscopes are divided into two main classes; realtime oscilloscopes and equivalent time oscilloscopes. Real-time samplers and highspeed analog-to-digital converters, ADCs, belong to the real time category. Typically, these scopes are available for frequency ranges up to hundreds of megahertz. Commercial availability is limited to a frequency of one gigahertz. For microwave frequencies, equivalent-time sampling oscilloscopes are needed. Typical commercial sampling oscilloscopes used in microwave TDNAs use Schottky diodes as sampling switches, $A P C-3.5 \mathrm{~mm}$ or $2.4 \mathrm{~mm}$ for its input and output connectors, $3 d B$ bandwidths from dc up to $50 \mathrm{GHz}$, and $10 \%-90 \%$ transition duration of the order of $7 \mathrm{ps}$. The dynamic range of the system is about $\pm 1 \mathrm{~V}$. For superconducting sampling oscilloscopes, Josephson junctions are used as sampling switches which operate in the cryogenic environment of $4.2^{\circ} \mathrm{K}$. Commercial 
superconducting oscilloscopes have $70 \mathrm{GHz}$ equivalent $9 \mathrm{~dB}$ bandwidth and 5 ps 10\%-90\% transition duration.

The majority of modern sampling oscilloscopes are equipped with a digitizing frame, which means that the oscilloscope system includes its own A/D and D/A converters and can output the acquired waveforms in a digital format. The quantizing error (including effective bits, noise, and nonlinearity) is also an important specification for the oscilloscope.

When selecting a sampling oscilloscope, several issues need to be considered. The bandwidth of the oscilloscope should meet the measurement needs. However, a wide bandwidth is not the only requirement for better performance. Since background noise increases proportionally with the oscilloscope bandwidth, larger or wide bandwidth means higher background noise implying degradation in measurement accuracy. If the $3 d B$ oscilloscope bandwidth is about the same as the DUT bandwidth, experts in the field consider this requirement suitable for measurements.

\subsubsection{The Computer}

A computer serves many functions in the operation of a TDNA system including: (1) data acquisition, (2) data storage, (3) Fourier transform computation, (4) computation of $S$-parameters or other desired parameters, and (5) presentation of the results in a useful tabulated or graphical forms. Currently, computers are becoming more and more powerful. To accomplish most of the desired functions, a 
personal computer (PC) is sufficient to accomplish the goal.

According to its functions, a TDNA can be configurated to perform TDR measurement, TDT measurement, or both. The intuitive nature of TDR and TDT techniques makes it possible to identify and locate discontinuities in interconnections and transmission lines such as IC packages, PC board traces, and connectors. Useful information such as the $S$-parameters of the DUT can be extracted from these signals, and hence, the system is referred to as a time domain network analyzer.

\subsection{HISTORY OF TDNA SYSTEM AND CALIBRATION}

\subsubsection{History of TDNA System}

Nicolson's paper on "Broad-band microwave transmission characteristics from a single measurement of the transmission characteristics from a single measurement of the transient response" [3] marks the beginning of the new era of time domain network analysis. Although time domain analysis can be traced to centuries ago, the first practically usable TDNA system was developed by Nicolson. This breakthrough was based on the following three basic facts: (1) the improvement introduced at the time to the sampling oscilloscope design, (2) the availability of the digital computer, (3) progress in signal processing theory.

In 1966, Grove at Hewlett-Packard Company developed a two-diode sampler in a Chapter 1. Introduction 
biconical cavity structure [4]. The new design increased the frequency bandwidth of sampling oscilloscopes from $4 \mathrm{GHz}$ up to $12.4 \mathrm{GHz}$ (or dc to X-band). Another oscilloscope company, Tektronix, introduced a six diode hybrid circuit sampler to match this bandwidth. The appearance of transistors in the 1950s and the integrated circuits (ICs) in the 1960s led to widespread use of the commerciallyavailable digital computer. The so-called mini-computers using transistors and ICs substituted the computers which were rather slow and required large power consumption and considerable "real estate" using vacuum tubes as switching elements. This progress tremendously increased the availability of computers and made complicated computations realistic.

This twin development sets a solid hardware background for the TDNA. Moreover, the signal processing field made a lot of progress during the same time period. In 1949, Shannon's sampling principle established a theoretical background for sampling and digitizing time domain waveforms [5]. In 1951, Samulon proposed a tabulated method to calculate the frequency spectrum from time domain transient response curves [6]. This made Fourier transform for the real experimental waveform possible although the accuracy of this method was low. In 1965, Cooley-Tukey published their famous algorithm to perform Fourier transform [7]. The algorithm reduced the computation time significantly. The multiplication operations are reduced from $N^{2}$ times to $N \cdot \log _{2} N$ times. This revolution first made the Fourier transform in a digital computer become a reality. For example, if $N=1024$, using the FFT algorithm would enhance the computation speed 100 times. 
In 1968, Nicolson presented the system and results of measurements of microwave components [3]. In his pioneer work, an HP-12 GHz sampling oscilloscope, an HP 1106A tunnel diode step generator, and a General Electric time-shared computer were used to form the TDNA system. In this system, two transmission lines were used to isolate multiple reflections between the scope and the DUT. The use of these line allowed a solution to the impedance mismatch problem between the scope and the DUT by means of gating in the time domain. Gating is a signal processing technique to acquire a desired time domain response through adjusting the beginning and the end of the waveform acquisition or signal processing epoch. In this work, Nicolson presented the measurement data for a few devices, like short-circuit stub, microwave directional coupler, and bandpass filter. Comparison between his TDNA, vector network analyzer (VNA) and theoretical calculations illustrated good agreement. Nicolson showed that a TDNA system can have a potential accuracy of about $\pm 0.1 \mathrm{~dB}$ and $\pm 1^{\circ}$.

In 1969, Stuckert developed a computer-augmented oscilloscope system (CAOS) [8]. The main interest of his research was a first step toward providing automation for the general laboratory experimenter. CAOS was designed to serve as an interface between the experimenter, the experiment, and the computer. It provided the function of digital acquisition of waveform data, data analysis, experiment control, and graphic and alphanumeric display.

Also in 1969, a time-domain data acquisition system was developed for the Atomic Energy Commission (AEC) by EG\&G [9]. This system was used to measure the response function of the scintillator and other subnanosecond phenomena. The 
exciting pulse, a $30 \mathrm{~A}$ and $50 \mathrm{ps}$ electron pulse, was generated by a linear accelerator. An HP sampling oscilloscope (12.4 GHz equivalent bandwidth) was used as the time domain receiver. A magnetic memory was used to store the digitized output. Averaging was used to increase the signal to noise ratio.

The National Bureau of Standards (NBS) began work on automating time-domain measurements in 1968. An HP sampling oscilloscope was modified to digitize and communicate with a Nova mini-computer. The design of the complete TDNA system as well as an evaluation of its performance up to $\mathrm{X}$ band was reported in 1973 [10], [11]. This NBS system is considered the first automatic TDNA system.

\subsubsection{History of TDNA Calibration}

The main features of early calibration techniques of the basic TDNA system involved the use of (a) a short circuit standard to produce a reference waveform, (b) two transmission lines as time domain isolators (to isolate secondary reflections due to impedance mismatch between the scope and DUT). In 1976, Riad established a model for the NBS' HP 1430 oscilloscope through physical modeling the sampling diodes and sampler structures [12]. The obtained model for the sampling oscilloscope is valuable not only for the TDNA application but also for the waveform metrology.

In 1987, Hewlett-Packard released the first of its HP 54120 scope series. Two main features characterize this series: (a) having a thin film planar geometry design for its wideband sampler and (b) having built in signal processing features which allow 
for waveform acquisition and processing enhancements. One of the main features introduced with the HP 54120 series is the time domain normalization feature. This normalization process uses standard short and load terminations to evaluate the system's performance to a normalized Gaussian excitation. The operator, within limits, can choose the transition duration of the Gaussian pulse. This approach does not provide a full TDNA calibration since it produces the response to a Gaussian pulse and not to an ideal impulse. Typically, the obtained response waveform has a clean (smooth) transition and hence is better used for transition duration computation as well as for further signal processing applications.

In contrast to earlier calibration work, this thesis is to develop a complete and generic calibration model for the TDNA system. The basic feature of this new work is establishing a complete system (error) model and evaluating the model parameters using a set of calibration standards. The work was inspired by the corresponding frequency domain techniques developed for VNA over the past 25 years. The emergence of this phase is based on the fact that traditional system calibration methods cannot satisfy the ever-increasing need of wider frequency band and higher accuracy for a TDNA system. As the measurement frequency increases, the skin effect of the transmission lines becomes severe. High frequency spectra are greatly attenuated by the two transmission lines which further reduces the $S / N$ at high frequencies. A critical situation arises if the longer length of transmission lines is needed to isolate multiple reflections. The main innovation of the second stage TDNA system is to remove the two transmission lines and introduce calibration techniques. This approach greatly alleviates the skin effect and improves the high frequency performance of the entire system. 
improves the high frequency performance of the entire system.

\subsection{DISSERTATION OBJECTIVES AND ORGANIZATION}

\subsubsection{Dissertation Objectives}

This dissertation is concerned with the calibration of TDNA systems. The approach used involves the development of a physically based model for the TDNA system. Model parameters are evaluated by measuring the TDNA's responses to known standard components. Measurements of several standards are required to accumulate enough knowledge to solve for the many unknown model parameters. Once the TDNA model parameters are known, de-embedding or deconvolution techniques can then be used to correct for the errors in network measurements due to these system.

For the sake of convenient presentation, and without any loss in generality, the HP 54120T TDR/Sampling oscilloscope was used as the TDNA system to demonstrate the calibration technique in this research. Although there are some other TDNA systems commercially available with much wider bandwidth, such as the HP 54124 $50 \mathrm{GHz}$ sampling oscilloscope combined with the Picosecond Pulse Labs PSPL 4015B 15 ps pulse generator, the concept of this calibration technique remains the same. 


\subsubsection{Dissertation Organization}

The dissertation is divided into eight chapters. Chapter 1 introduces the typical time domain network analyzer system, its configuration, and its history. The functions and requirements for different parts in the system are also presented in this Chapter. Chapter 2 deals with the modeling method which includes the evolution from a time domain system to a scatter parameter model. The chapter also introduces a technique to evaluate the model parameters from the measurements. Chapter 3 concentrates on the calibration standards. It outlines the unique feature of the time domain approach. It also introduces the requirements for the time domain standards and illustrates how to select them. In Chapter 4, the data processing for the time domain system including averaging, truncation and Fourier transform are presented. System error analysis is the subject of Chapter 5. In this very important chapter, the most significant error sources are discussed. Statistics and error analysis are discussed to achieve a complete picture of the errors. Chapter 6 shows the obtained experimental results. Examples of the system applications demonstrate the validity of this calibration technique. In the Conclusions chapter, Chapter 7, a summary of the research and progress is provided. In the Appendices, equations for simplifying the system model and formulas for modeling the open circuit standard are discussed. 


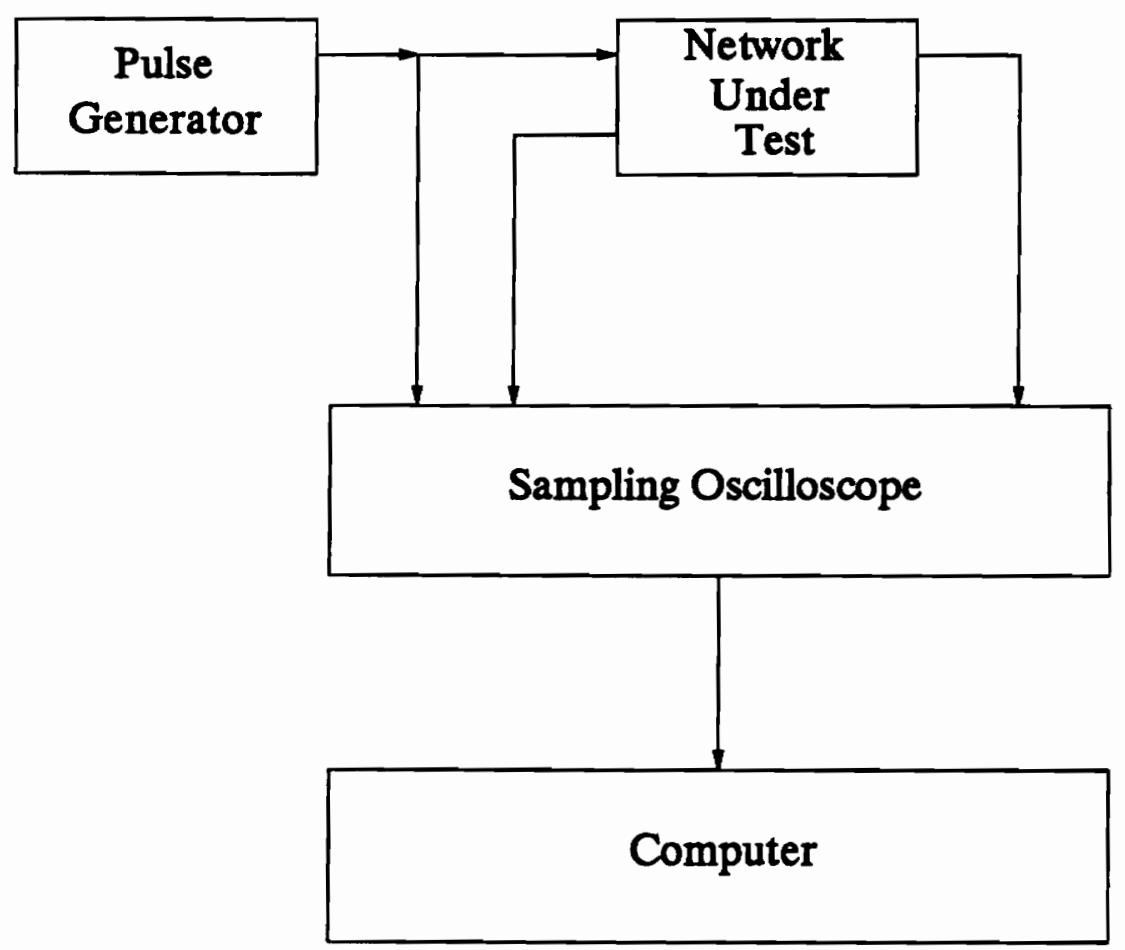

Figure 1.1.1 A block diagram of TDNA system. 


\section{CHAPTER 2}

\section{MODELING THE TIME DOMAIN NETWORK ANALYZERS}

\subsection{INTRODUCTION}

Typically, instrument calibration techniques require either (a) having a calibrated instrument (of the same kind) to which the performance of the instrument to be calibrated is compared, or (b) having known (standard) measurables which are used to identify instrument characteristics pertinent to its calibration. The latter approach requires that an error model for the instrument is available and that adequate number of standards be available to fully evaluate all necessary model parameters.

For TDNA systems, one TDNA can be used to calibrate another if, and only if, it has a much wider bandwidth. The error in such calibration procedure depends on ratio between the two bandwidths. This approach has the following deficiencies: (a) it cannot be applied to the state of the art instruments since they would posses widest band available; (b) it would result in significant errors in the calibration of most wideband TDNAs since the bandwidths are in the same order. On the other hand, the modeling approach is limited by the following factors: (a) development of 
a proper model for the system and its error components and sources; (b) the availability of "measurable" standards adequately characterized for the evaluation of the model parameters. Based on the above discussion, it is evident that the modeling approach is best suited for the TDNA calibration.

As discussed in Chapter 1, the proposed calibration technique involves the development of a physically-based model for the TDNA measurement system. Measurements of known standards can then be used to determine the TDNA model parameters. Knowing the model parameters of the TDNA system, it then becomes possible to use this model to obtain accurate network analyzer measurements through de-embedding of TDNA contribution to these measurements.

A precise and accurate model for the system is vital because the measurement data cannot be correctly interpreted without a correct system model. The modeling process includes two steps; first, establishing a model for the system, and second, evaluating the model parameters. In order to establish a good model for the system, first we need to fully understand the operating principle of the system, its key components, circuits, and devices. This understanding is necessary to provide the basis for establishing a physically-based model. Such a model is typically complicated and needs to be simplified before the evaluation step. The simplification step leads to the reduction in the number of model parameters to be evaluated and thus reduces the complexity of the evaluation step. This chapter is devoted to a discussion of approach followed in establishing the system model, while the evaluation step will be the subject of future chapters. 


\subsection{TDNA SPECIFICATIONS AND OPERATION PRINCIPLE}

As discussed in Chapter 1, the HP 54120T Sampling Oscilloscope/TDR system was used as the TDNA system in consideration in this research. Although some of the discussions which follow a specific to the HP system, it will become clear later on that the proposed technique is general in its applicability to other TDNA systems. First, it is important to relate to the system's specification as well as its principle of operation. This is the subject of this section.

\subsubsection{System Specifications}

The HP 54120T system's pulse generator uses step recovery and Schottky diodes to produce a relatively flat and fast step-like pulse. The manufacturer specifies that the pulse has $0.2 \mathrm{~V}$ amplitude and about $35 \mathrm{ps}$ transition duration and the top level is flat within $\pm 1 \%$ [13]. The sampling network uses the same GaAs technology used in the HP 8510 Network Analyzer. Its switching time is less than $20 \mathrm{ps}$ and its equivalent frequency bandwidth is around $20 \mathrm{GHz}$. The oscilloscope has $0.25 p s$ time resolution and $10 p s$ accuracy. For the vertical channel, it has 32 $\mu V$ resolution (average mode) and $0.4 \%$ vertical accuracy. The vertical sensitivity is $1 \mathrm{mV} / \mathrm{div}$. The resultant transition duration of the TDR and TDT system, including the effects of both the pulse generator and sampler is about $45 \mathrm{ps}$, which corresponds to about $8 \mathrm{GHz}$ system bandwidth. 


\subsubsection{TDNA Principles of Operation}

Figure 2.2.1 shows the conceptual block diagram of the HP-54120T in the TDNA configuration, while Fig. 2.2.2 shows the corresponding schematic. The shown diagram demonstrates the typically system configuration used in the HP 54120 series. This configuration uses an internal feed-through pulse generator in Channel 1 and terminating samplers in all channels. For full TDR/TDT measurement using the internal pulse generator, one would use Channel 1 for TDR and any other channels, e.g. Channel 2 for TDT. An extension cable is typically needed to allow the insertion of the DUT between the two channels for TDT measurements. This cable is to be considered as an integral part of Channel 2 through the calibration and measurement procedures.

Referring to the schematic, an "on-off" ideal trigger pulse is assumed to trigger and synchronize the timing systems of the operation. The trigger pulse starts the measurement cycle by triggering the pulse generator and producing a step like voltage pulse. This pulse travels in two possible directions. One direction is through the transmission path " $b$ " to the sampler (initial pulse) and the other through the transmission path "a" to the TDR port (Port 1), while reflections from the TDR port travel back to reach the sampler at a delayed time relative to that of the arrival of the initial pulse. Signals arriving to the sampler are then sampled and detected by the sampling gate. The sampler's strobing pulse is synchronized to the trigger pulse. The detected waveform is then digitized by the analog to digital A/D converter into digital data which is then fed to the oscilloscope and the subsequent signal processing unit. In the meantime, the transmitted step passes 
through the path "c" to reach the sampling gate of Channel 2. It will also be digitized and displayed on the oscilloscope screen. Useful information about the DUT can then be extracted from both the reflection and transmission.

\subsection{SYSTEM $S$-PARAMETER MODEL}

An $S$-parameter model for the system is developed and shown in Fig. 2.3.1. In this model, $S_{p}, S_{a}, S_{b}, S_{c}, S_{d 1}$ and $S_{d 2}$ are the $S$-parameters for network sections denoted $p, a, b, c, d 1$ and $d 2$, respectively in Fig. 2.2.2. In this model, $E_{g}, W_{r}$ and $W_{t}$ are as follows.

$E_{g}$ is the frequency response of the exciting pulse. For convenience, either the ideal unit step signal $u(t)$ or impulse signal $\delta(t)$ is used.

$W_{r}$ is the frequency response of the reflected voltage wave as detected from the oscilloscope displayed waveform.

$W_{t}$ is the frequency response of the transmitted voltage wave as detected from the oscilloscope displayed waveform.

Using signal flow graph reduction techniques, this model is reduced to that of Fig. 2.3.2. The relation of the model parameters between the simplified model and the original model is given in Appendix A. The reduction is performed for two reasons: (a) the model of Fig. 2.3.1 has more unknown terms than needed to establish the calibration, and (b) the configuration of Fig. 2.3.2 is generic and would apply to TDR and TDT network analyzers in general. 
To provide some physical insight into the $S$-parameters of the model, the following summarizes the primary components of the individual parameter (a first order approximation):

$S_{g r}:$ frequency domain form of the acquired sampler's direct response to the pulse generator signal,

$S_{g l}:$ frequency domain form of the the pulse generator signal measured at the calibration plane of Channel 1 ,

$S_{1 r}:$ frequency domain form of the acquired sampler's direct response to the signal reflected into Channel 1 ,

$S_{11}$ : the reflection coefficient at the calibration plane of Channel 1,

$S_{2 t}$ : frequency domain form of the acquired sampler's response to the signal transmitted into Channel 2 and

$S_{22}$ : the reflection coefficient at the calibration plane of Channel 2.

In an ideal TDR and TDT system (ideal pulse and ideal sampler), $S_{g r}, S_{g 1}, S_{1 r}$ and $S_{2 t}$ would all equal to one, while, $S_{11}$ and $S_{22}$ would be both zeros.

\subsection{MODELS FOR ALTERNATE TDNA SYSTEM CONFIGURATIONS}

There are other possible configurations that are typically used in available TDNA systems. Two of these configurations will be discussed in this section in order to demonstrate the applicability of the proposed modeling scheme. The external pulse generator with terminating samplers is one configuration and the other is external pulse generator with a feed-through sampler. 
In the first configuration, an external pulse generator is used with terminating sampler such as those of the HP 54120 series. In this case a signal splitter (power splitter or matched $\mathrm{T}$ junction) is used to provide a 3 way connection between the pulse generator, the DUT and the TDR sampler. Figure 2.4.1 demonstrates the configuration, its model, as well as shows that it can be simplified into the same form of Fig. 2.3.2.

The second common configuration is that of the feed-through sampler. This configuration was commonly used by earlier HP and Tektronix systems. In this configuration, see Fig. 2.4.2, an external pulse generator is used with the feed through sampler with no need for a power splitter. Again, modeling this configuration as demonstrated in the figure leads to the same simplified model of Fig. 2.3.2.

\subsection{EVALUATION OF MODEL PARAMETERS}

The previous section demonstrated that a TDNA system can be modeled as an $S$ parameter model of the form given in Fig. 2.3.2. This model has six unknown parameters (four on the TDR side and two on the TDT side). If fully known, the system would be considered fully calibrated. In other words, if all six parameters are evaluated, the measured waveforms $w_{r}(t)$ and $w_{t}(t)$ can be used to accurately determine the $S$-parameter of the measured DUT.

To evaluate the model parameters, known impedances (reflection standards) are to 
be used. Examining the model for a TDR measurement with a reflection standard terminated at Channel 1, see Fig. 2.5.1, we can write the relationship for the TDR model

$$
\frac{W_{r}}{E_{g}}=S_{g r}+\frac{S_{g 1} S_{1 r} \Gamma}{1-S_{11} \Gamma}
$$

where $\Gamma$ is frequency domain reflection coefficient of the standard. This equation displays only three unknowns on the TDR side. $\mathrm{S}_{g r}, \mathrm{~S}_{g !} S_{1 r}$, and $S_{11}$.

On the TDT side, if a direct connection between the two channels is provided (without additional devices), the following terminal relationships result for the TDR model

$$
\frac{W_{r}}{E_{g}}=S_{g r}+\frac{S_{g 1} S_{1} S_{22}}{1-S_{11} S_{22}}
$$

and for the TDT model

$$
\frac{W_{t}}{E_{g}}=\frac{S_{g 1} S_{2 t}}{1-S_{1 t} S_{22}}
$$

which displays two additional unknowns, $\mathrm{S}_{g t} S_{2 t}$ and $S_{11} S_{22}$.

It is apparent from the above discussion that only five unknowns need to be determined to fully calibrate the TDNA system and that five independent measurements (three for the TDR model and two for the TDT model) are required to determinate the five unknowns. The three TDR model measurements imply the 
need for three different $\Gamma$ values for each measurement. For the TDT model, it is clear that the connection of Fig. 2.5.2 results in two independent waveforms $w_{r}(t)$ and $w_{t}(t)$ leading to the evaluation of the two TDT model unknowns.

The following discussion, however, provides a confirmation to the apparent observation that only three $\Gamma$ measurements are needed to evaluate the TDR model.

Examining equation (2.5.1), we notice that there are four model parameters $S_{g r}$, $S_{g 1}, S_{1 r}$ and $S_{11}$. Since the equation is a non-linear equation with respect to these unknowns, it is not obvious to judge how many independent variables exist in that equation. The equation needs to be linearized first before we are certain that only three unknowns are needed. Rearranging equation (2.5.1), we obtain

$$
\mathrm{S}_{g r}-S_{g r} S_{11} \Gamma+\frac{W_{r}}{E_{g}} S_{11} \Gamma+S_{g 1} S_{1} \Gamma=\frac{W_{r}}{E_{g}}
$$

In order to eliminate the product terms in equation (2.5.3), we introduce $T$ parameters. The transforming relations are

$$
\begin{aligned}
& \mathrm{S}_{g r}=\frac{T_{12}}{T_{22}} \\
& \mathrm{~S}_{1 r}=T_{11}-\frac{T_{12} T_{21}}{T_{22}}, \\
& \mathrm{~S}_{g 1}=\frac{1}{T_{22}}
\end{aligned}
$$


$\mathrm{S}_{11}=-\frac{T_{21}}{T_{22}}$

Substituting equations (2.5.4) $-(2.5 .7)$ into equation (2.5.3) and rearranging it, we obtain

$$
T_{11}+\Gamma T_{12}-\frac{W_{r}}{E_{g}} T_{21}-\frac{W_{r}}{E_{g}} \Gamma T_{22}=0
$$

If we were to assume that a set of four calibration standards with reflection coefficients $\Gamma_{1}, \Gamma_{2}, \Gamma_{s}$ and $\Gamma_{4}$ are needed to determine the four unknown $T \mathrm{~s}$. Then, substituting these $\Gamma \mathrm{s}$ into equation (2.5.8), we obtain a set of linear equations as follows:

$\left[\begin{array}{cccc}1 & \Gamma_{1} & \frac{W_{r 1}}{E_{g}}-\frac{W_{r 1}}{E_{g}} \Gamma_{1} \\ 1 & \Gamma_{2} & \frac{W_{r 2}}{E_{g}}-\frac{W_{r 2}}{E_{g}} \Gamma_{2} \\ 1 & \Gamma_{3} & \frac{W_{r s}}{E_{g}}-\frac{W_{r s}}{E_{g}} \Gamma_{s} \\ 1 & \Gamma_{4} & \frac{W_{r 4}}{E_{g}}-\frac{W_{r 4}}{E_{g}} \Gamma_{4}\end{array}\right]\left[\begin{array}{c}T_{11} \\ T_{12} \\ T_{21} \\ T_{22}\end{array}\right]=\left[\begin{array}{c}0 \\ 0 \\ 0 \\ 0\end{array}\right]$

It is easy to see that the rank of the $4 \times 4$ matrix is less than four or else the equations only have trivial zero solutions. If we divide equation (2.5.8) both sides by $T_{22}$ and rearrange it, we obtain

$\frac{T_{11}}{T_{22}}+\Gamma \frac{T_{12}}{T_{22}}-\frac{W_{r}}{E_{g}} \frac{T_{21}}{T_{22}}=\frac{W_{r}}{E_{g}} \Gamma$

From equation (2.5.10), we have 


$$
\left[\begin{array}{ccc}
1 & \Gamma_{1} & -\frac{W_{r 1}}{E_{g}} \\
1 & \Gamma_{2} & -\frac{W_{r 1}}{E_{g}} \\
1 & \Gamma_{s} & -\frac{W_{r g}}{E_{g}}
\end{array}\right]\left[\begin{array}{l}
\frac{T_{11}}{T_{22}} \\
\frac{T_{12}}{T_{22}} \\
\frac{T_{21}}{T_{22}}
\end{array}\right]=\left[\begin{array}{l}
W_{r 1} \\
E_{g} \\
W_{r 2} \\
\frac{E_{g}}{E_{g}} \\
W_{r s} \\
\frac{E_{g}}{r_{g}}
\end{array}\right] .
$$

Unless $\Gamma_{1}, \Gamma_{2}$, and $\Gamma_{3}$ are not independent, equation (2.5.11) can have a unique solution. This confirms that there are only three independent parameters in the TDR model. Actually, the three parameters are $S_{g r}, S_{11}$, and the product $S_{g 1} S_{1 r}$

In summary, a set of three standards with known characteristics is needed to obtain the TDR model parameters. Using three standard terminations with known reflection coefficients, $\Gamma_{1}, \Gamma_{2}$ and $\Gamma_{s}$, three TDR responses $W_{r 1}, W_{r 2}$ and $W_{r s}$ are measured, respectively. The TDR model parameters can then be determined as follows:

$S_{11}=\frac{W_{r 1}\left(\Gamma_{s}-\Gamma_{2}\right)+W_{r 2}\left(\Gamma_{1}-\Gamma_{3}\right)+W_{r s}\left(\Gamma_{2}-\Gamma_{1}\right)}{\Gamma_{1} W_{r 1}\left(\Gamma_{3}-\Gamma_{2}\right)+\Gamma_{2} W_{r 2}\left(\Gamma_{1}-\Gamma_{s}\right)+\Gamma_{s} W_{r s}\left(\Gamma_{2}-\Gamma_{1}\right)}$,

$S_{g r}=\frac{\left(W_{r 1} \Gamma_{2}-W_{r 2} \Gamma_{1}\right)-S_{11} \Gamma_{1} \Gamma_{2}\left(W_{r 1}-W_{r 2}\right)}{E_{g}\left(\Gamma_{2}-\Gamma_{1}\right)}$

$S_{g 1} S_{1 r}=\left(\frac{W_{r 1}}{E_{g}}-S_{g r}\right)\left(\frac{1}{\Gamma_{1}}-S_{11}\right)$

For the transmission part of the model, TDT, only two unknown parameters exist as apparent from (2.5.2). The two unknowns are $S_{91} S_{2 t}$ and $S_{11} S_{22}$. The proposed calibration technique does not require any additional standards for determining Chapter 2. Modeling the Time Domain Network Analyzers 
these two unknowns. Using the calibrated TDR channel, a direct connection of the TDT channel (including the extension cable) to the TDR channel would allow the determination of both unknowns. Denoting the corresponding reflection and transmission signals for these measurements as $W_{r 4}$ and $W_{t}$, the unknowns of the TDT model take the form

$S_{11} S_{22}=\frac{\frac{W_{r 4}}{E_{g}}-S_{g r}}{S_{g 1} S_{1 r}+S_{11}\left(\frac{W_{r 4}}{E_{g}}-S_{g r}\right)} S_{11}$,

and

$S_{91} S_{2 t}=\frac{W_{t 4}}{E_{g}}\left(1-S_{11} S_{22}\right)$

Once all model parameters are determined, they can be used to de-embed the responses of the TDR and TDT systems from the reflection and transmission waveforms to determine the response of the DUT due to an ideal step or impulse input $e_{g}(t)$. The de-embedded $S$-parameters for the DUT are obtained from TDR and TDT measurements on the DUT, while connected in forward as well as backward directions. Four sets of measurement data are then available which are used to solve for the four $S$-parameters of th DUT. The accuracy of these deembedded results is largely dependent on the accuracy of the parameters of the standards used. 


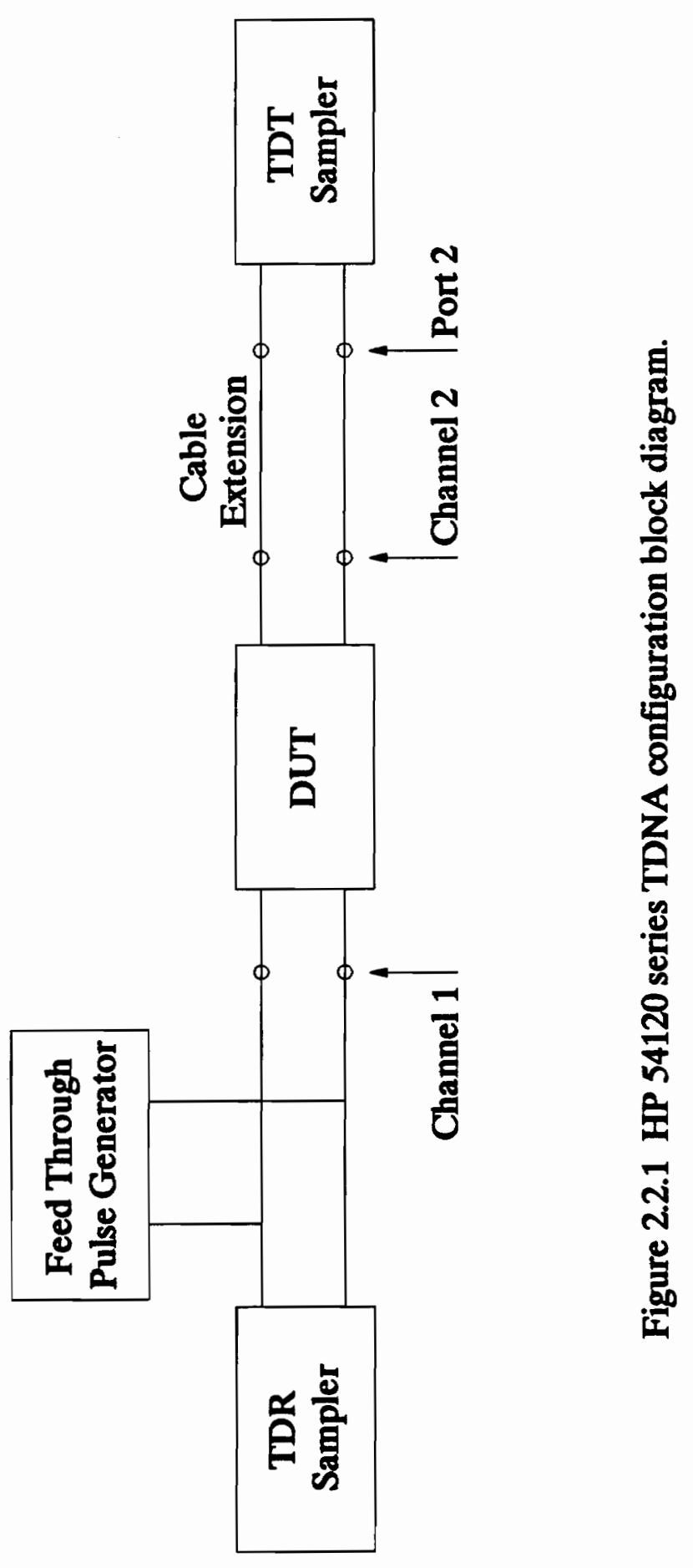




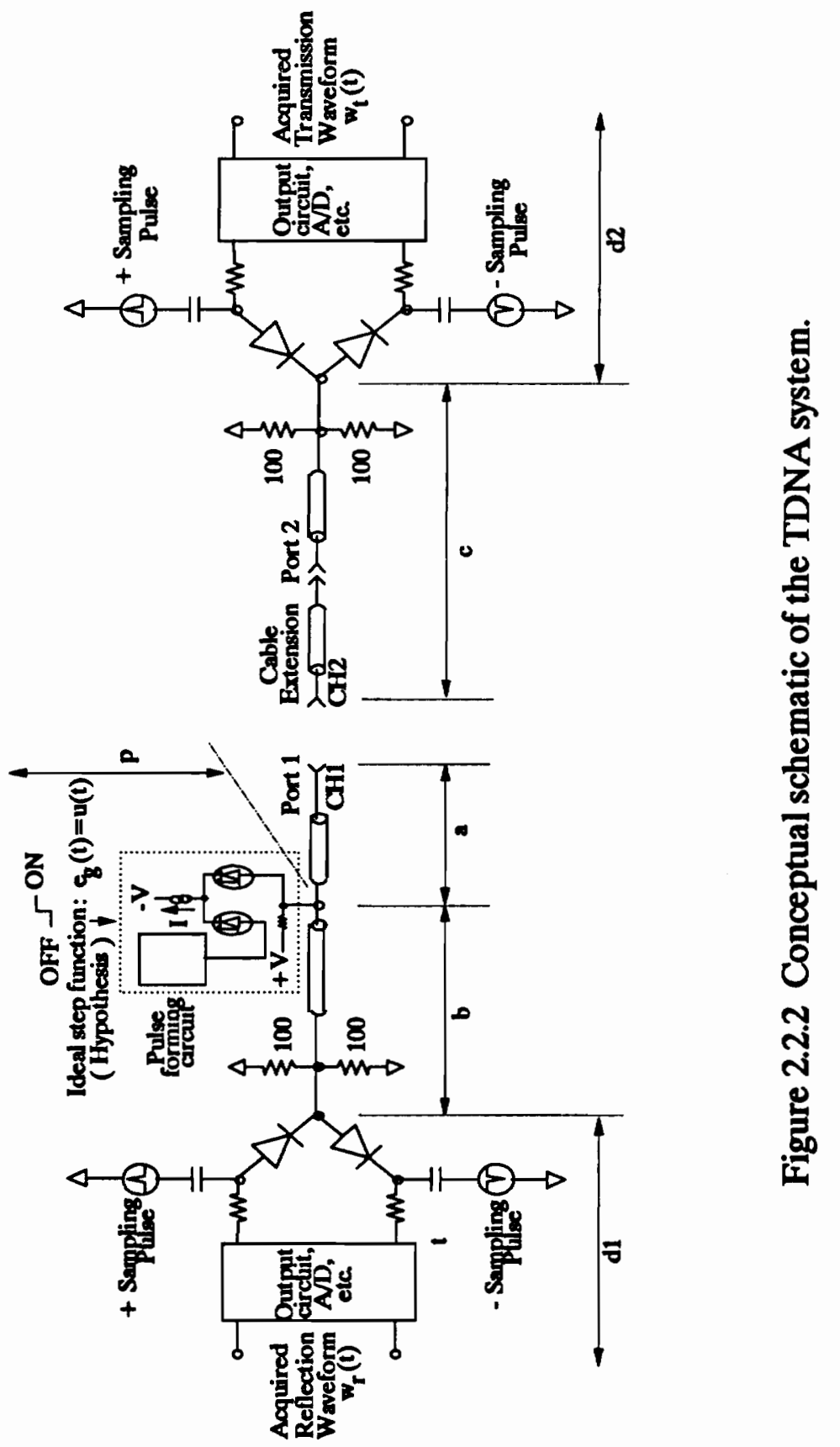




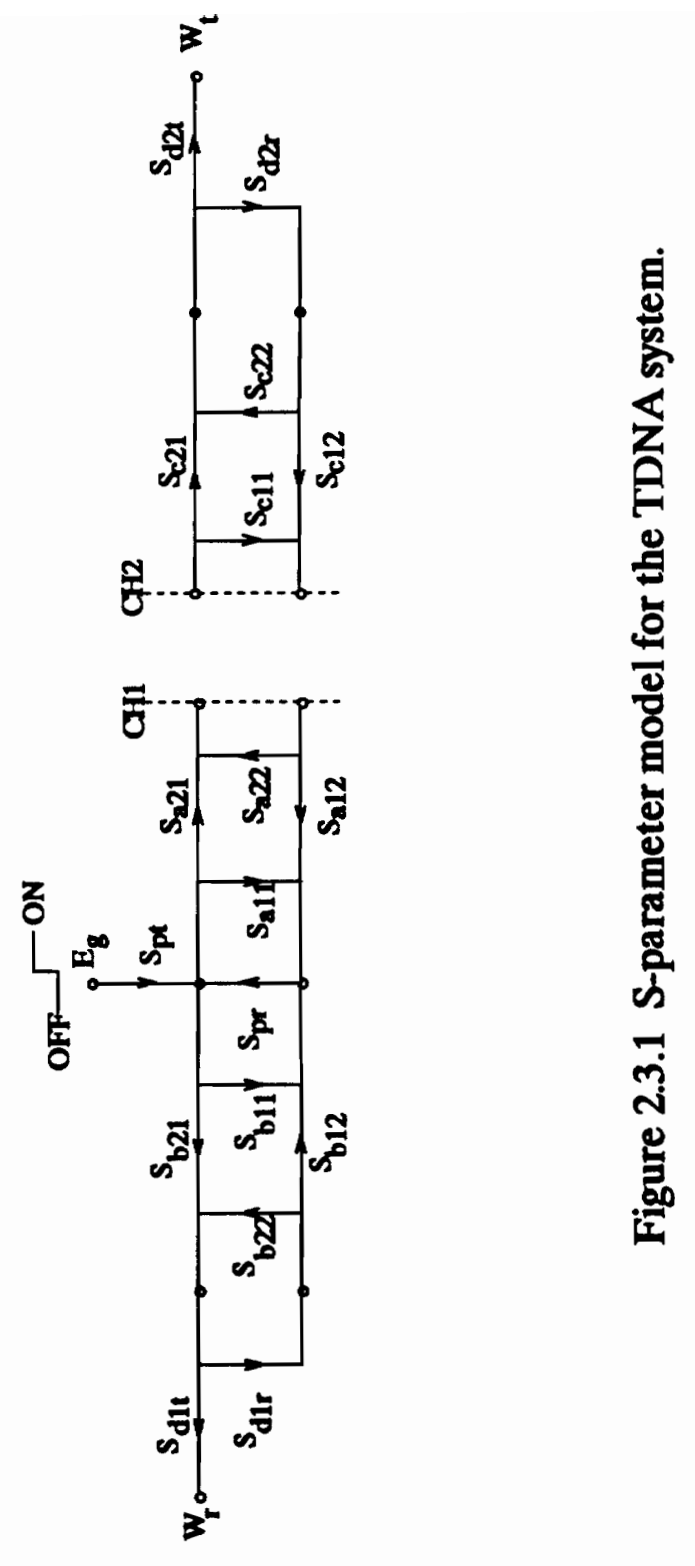



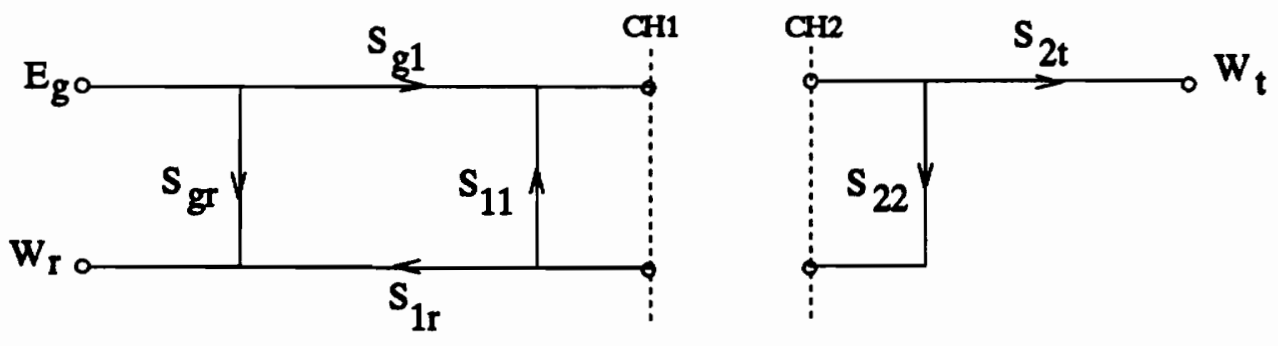

Figure 2.3.2 Simplified S-parameter model. 


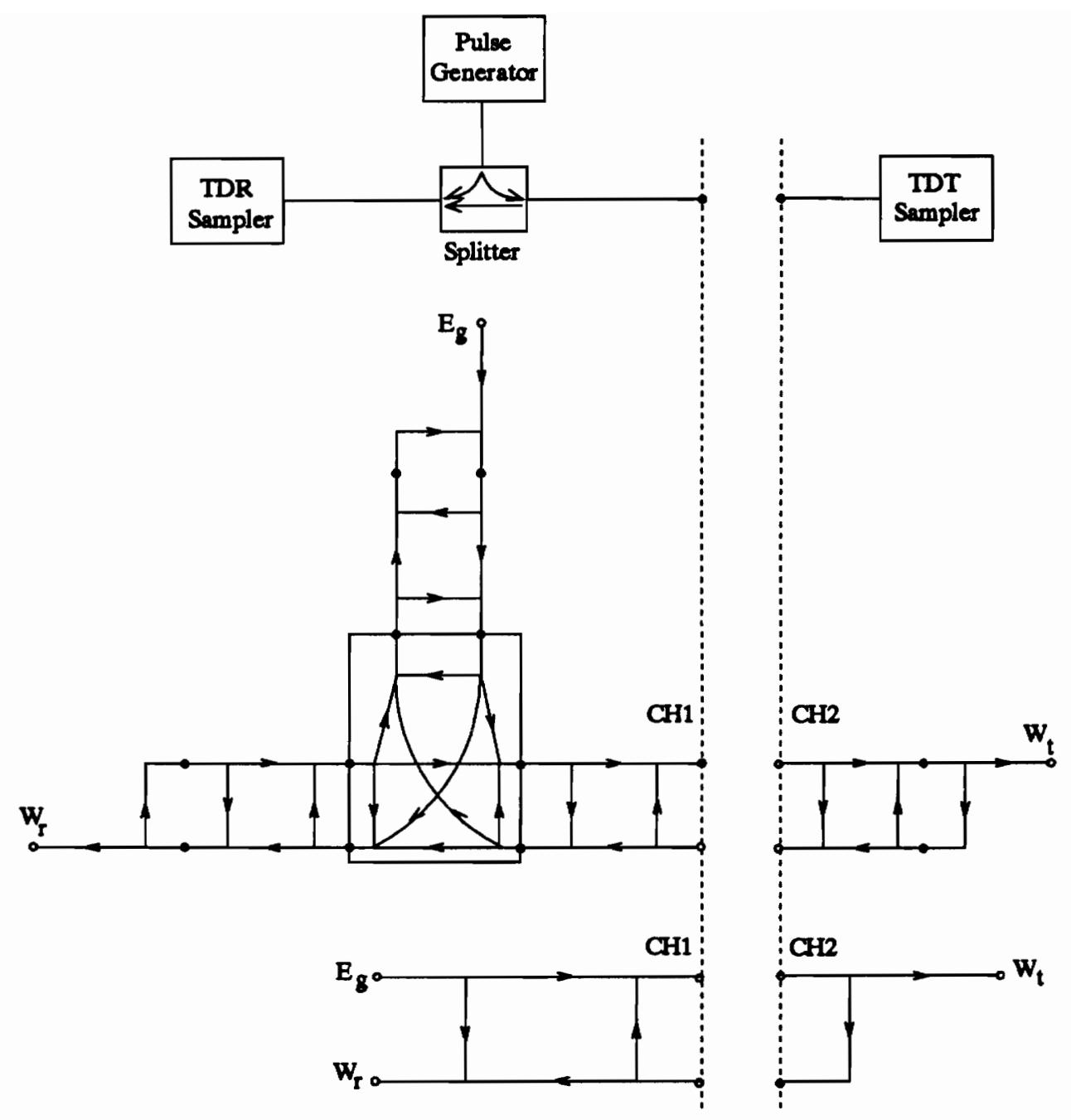

Figure 2.4.1 Modeling the EXT pulse generator with power splitter configuration. 


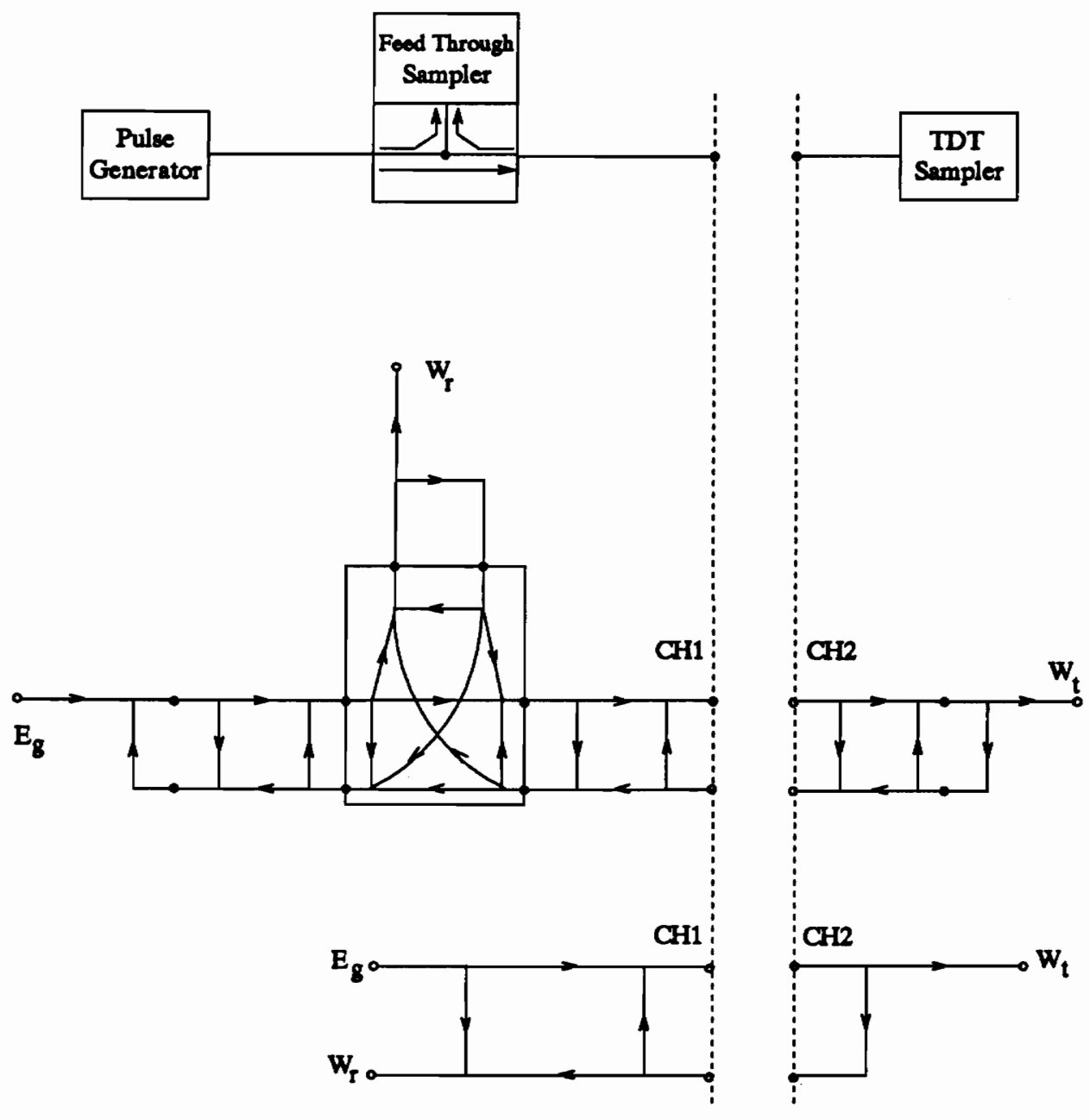

Figure 2.4.2 Modeling the feed through sampler configuration. 


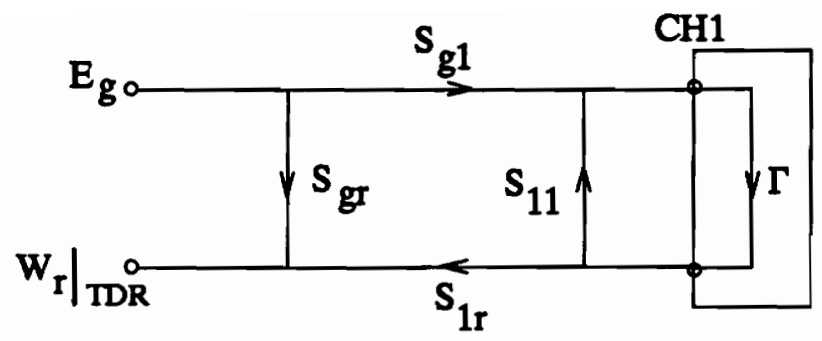

Figure 2.5.1 The TDR model evaluation setup. 


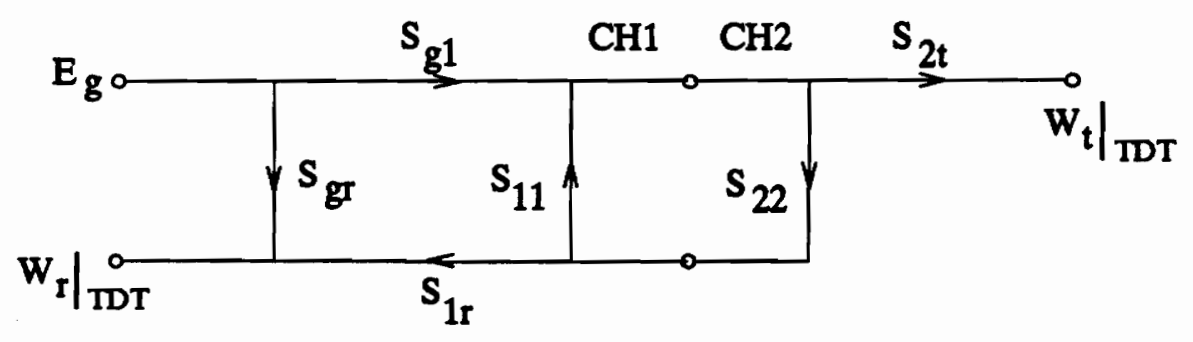

Figure 2.5.2 The TDT model evaluation setup. 


\section{CHAPTER 3}

\section{TIME DOMAIN CALIBRATION STANDARDS}

\subsection{INTRODUCTION}

A standard is a physical device having stable, precisely defined characteristics, that is used as a reference for a unit of measurement [14]. For our calibration, we need a reflection or impedance standard. It is relevant to state that TDNA systems have different requirements and characteristics from those of frequency domain vector network analyzers (VNA). Consequently, impedance standards available for calibration of VNA may not directly usable for the time domain system and vice versa. In this regard, it is important to realize that unlike the case for the VNA, the bandwidth for TDNA calibration standard should be much larger than the system's nominal bandwidth. The reason for that is explained as follows. Assuming that both a TDNA and a VNA have the same nominal bandwidth, the VNA does not measure any spectrum information beyond the bandwidth. In contrast, the TDNA waveforms would have a significant spectrum even at double or triple the nominal bandwidth frequency.

Such bandwidth requirement may be hard to realize for an impedance standard, in 
particular those of load terminations. However, due to the ability to gate time domain waveforms, a coaxial precision line can simulate a known impedance over a relatively wideband of frequencies. Gating in the time domain enables the exclusion of undesired waveform responses by adjusting the acquisition or signal processing time epoch. Using this feature, it becomes possible to gate out the reflections of the far end of a transmission line terminating the TDR port. By doing so, it becomes possible to simulate the response of an infinitely long transmission line using a line of sufficient length that corresponds to a delay greater than that of the time gate (epoch). This enables us to use such a transmission line section to simulate an ideal impedance standard in the time domain.

As discussed above, a matched load can be achieved using a coaxial line and the time domain gating feature. The short circuit and open circuit terminations are available commercially since they are usable in VNA calibration. A caution is to be cited here since the available reflection standards are offset ones, see Figs. 3.1.1 and 3.1.2. The two standards have an additional section of precision air line as the "offset" due to mechanical considerations.

For all three standards completely known over the wideband of frequency of interest, three main elements need to be characterized, the actual short, the actual open and the coaxial line. Such characterization is best achieved based on physical models of the devices including the knowledge of their geometrical structures and properties of materials. In other words, the standards' $\Gamma$ values are calculated from their physical structures. Such standards are typically referred as calculable standards. 
In this chapter, we will first discuss the choice of an optimum standard set for TDNA calibration. Next, we will discuss the realization for these standards keeping in mind the nature and characteristics of time domain measurement systems. Finally, we plan to discuss error sources in the chosen standards.

\subsection{OPTIMUM STANDARD SET FOR TDNA CALIBRATION}

As discussed earlier, three known $\Gamma$ values are needed to evaluate three unknowns in the TDR model. To determine the optimum values for the three $\Gamma \mathrm{s}$, a sensitivity analysis is in order. Minimum sensitivity is desired to accurately determine the three unknowns, and hence we take the derivatives of $S_{g r}, S_{11}$ and $S_{g 1} S_{1 r}$ with respect to $\Gamma$ as follows,

$\frac{\partial S_{g r}}{\partial \Gamma}=-\frac{S_{g 1} S_{1 r}}{\left(1-S_{11} \Gamma\right)^{2}}$

$\frac{\partial S_{11}}{\partial \Gamma}=-\frac{1}{\Gamma^{2}}$

$\frac{\partial S_{g l} S_{1 r}}{\partial \Gamma}=-\frac{1}{\Gamma^{2}}\left(\frac{W}{E_{g}}-S_{g r}\right)$

The smaller the derivative values are, the less sensitive the $S$-parameters of the model respect to the standards would be. This means low errors in evaluating the model parameter can be achieved by minimizing the derivatives. Examining equations (3.2.2) and (3.2.3), it becomes clear that $\Gamma^{2}$ needs to be maximized to meet the above criteria. The largest possible values for $\Gamma^{2}$ is 1 implying $\Gamma$ values of 
+1 and -1 . This means that $\Gamma=+1$ and $\Gamma=-1$ are two optimum choices in this regard. From equation (3.2.1), we need to maximize $1-S_{11} \Gamma$, thus the optimum $\Gamma$ value is $\Gamma=-S_{11}$. The symbol " $*$ " signifies the complex conjugate operation. $S_{11}$, however, is an unknown parameter whose value must be determined. However, even if we know $S_{11}$ as a function of frequency, it is almost impossible to develop a standard whose $\Gamma$ equals to $-S_{11}^{*}$ over the wide frequency band of interest. If $\Gamma$ is not equal to $-S_{11^{*}}$ and not equal to zero, as the frequency changes, the denominator at some frequencies will be larger than one, at other frequencies less than one as shown in Fig. 3.2.1 and hence high sensitivities will occur over segments of the frequency band. Selecting $\Gamma=0$ is a realistic choice for two reasons, the first is that $S_{11}$ is very small any way, and the second, $\Gamma=0$, it offers a constant value for $1-S_{11} \Gamma$ over the entire frequency. Although this choice is not as good as the $-S_{11^{*}}$ at certain frequencies, it is much better than the non-zero at other frequencies. In this sense, it is a compromised optimum value.

To summarize, a realistic set of optimum calibration standards is $\Gamma=0,+1$ and -1 . The three standards, $\Gamma=0,+1$, and -1 , correspond to a matched load, an open circuit, and a short circuit, respectively.

\subsection{COAXIAL TRANSMISSION LINE AS A CALCULABLE STANDARD}

A coaxial transmission line and its equivalent circuit are shown in Fig. 3.3.1. The characteristic impedance $\left(Z_{0}\right)$ of a transmission line can be expressed by the following general relationships [15]:

Chapter 3. Time Domain Calibration Standards 
$Z_{0}=\sqrt{(R+j \omega L) /(G+j \omega C)}$,

where:

$Z_{0} \equiv$ the characteristic impedance of the coaxial transmission line,

$R \equiv$ total resistance per unit length of both conductors,

$L \equiv$ total inductance per unit length of both conductors,

$G \equiv$ distributed conductance per unit length between the conductors and

$C \equiv$ distributed capacitance per unit length between the conductors.

Although an infinite number of electromagnetic field configurations or modes can propagate along a coaxial transmission line, the mode we are almost always interested in is the principal or transverse electromagnetic mode (TEM) because, except in rare instances, coaxial lines are intended to operate in this mode. If the conductor radii of the line are $a$ and $b$ as shown in Fig. 3.3.1, under quasi-static assumption, the $R L C G$ line parameters can be estimated as follows.

\section{Capacitance per unit length $C$}

$C=\frac{2 \pi \epsilon}{\ln \left(\frac{b}{a}\right)}$

Conductance per unit length $G$

$G=\frac{2 \pi \sigma_{d}}{\ln \left(\frac{b}{a}\right)}$, with $\sigma_{d} \simeq 0$ for air dielectric, i.e.

$G=0$.

Chapter 3. Time Domain Calibration Standards 
Total inductance per unit length $\underline{L}$

The external inductance per unit length $l_{e}$ is

$l_{e}=\frac{\mu}{2 \pi} \ln \left(\frac{b}{a}\right)$.

At high frequencies, using the planar skin effect model, the internal inductance per unit length is

$l_{i}=\sqrt{\frac{\mu}{2 \omega \sigma}}\left(\frac{1}{2 \pi a}+\frac{1}{2 \pi b}\right)$,

where

$$
\begin{aligned}
& \sigma \equiv \text { conductivity of the inner and outer conductor material and } \\
& \mu \equiv \text { permeability of the inner and outer conductor material. }
\end{aligned}
$$

The total inductance per unit length is

$$
L=l_{e}+l_{i}=\frac{\mu}{2 \pi} \ln \left(\frac{b}{a}\right)+\sqrt{\frac{\mu}{2 \omega \sigma}}\left(\frac{1}{2 \pi a}+\frac{1}{2 \pi b}\right)
$$

Total resistance per unit length $\underline{R}$

At high frequencies, using the planar skin effect model, the total resistance per unit length is

$$
R=\sqrt{\frac{\omega \mu}{2 \sigma}}\left(\frac{1}{2 \pi a}+\frac{1}{2 \pi b}\right) .
$$


Since the coaxial air lines have a limited length, in time domain, this interprets a corresponding limited time epoch. The time window may be increased by increasing the line length which, however, is often limited by the mechanical manufacturing. A frequency domain interpolation can also be used to extend the time window. The only assumption for this method is that there is no sharp resonance in the interested frequency range. This is the case for our transmission line standards.

\subsection{DISCONTINUITY CAPACITANCE OF COAXIAL DISCONTINUITY}

The equations of Section 3.3 show that the characteristic impedance of a coaxial air line is determined by the ratio of its inner conductor dimension $a$ to its outer conductor dimension $b$. Varying this ratio leads to the different characteristic impedance or different impedance standard. From the mechanical consideration, changing $a$ is more convenient and easier to be realized. However, connecting two coaxial air lines with different dimensions results in a mechanical discontinuity at the interface, such as in the case of changing the conductor size $a$ to obtain different characteristic impedance. Typically, this mechanical discontinuity is modeled by a shunt capacitance. Assuming that the inner diameter of the outer conductors is $R$ and the diameters of two inner conductors are $r_{1}$ and $r_{2}$, respectively, two parameters, $\alpha$ and $\tau$, related to the discontinuity can be defined as $\alpha=\frac{R-r_{2}}{R-r_{1}}$ and $\tau=\frac{R}{r_{1}}$. Table 3.4 .1 shows the computed values of the discontinuity capacitances [16]. They were obtained by taking into account the first 40 higher order modes of propagation, and extrapolating hyperbolically the 
returned values of step capacitances as a function of numbers of modes used to produce them, and regarding the asymptotic value of this hyperbola as the final answer.

\subsection{COAXIAL SHORT AND OPEN CIRCUIT STANDARDS}

Using the knowledge of coaxial air transmission lines, the frequency domain offset short and offset open standards can be well modeled over a much wider frequency range which can match the time domain system.

\subsubsection{Coaxial Short Circuit Standard}

Theoretically, the discontinuity short can be modeled as a resistor and inductor in series. Over the frequency range of interest, however, it can be approximated as zero impedance, as shown in Fig. 3.1.1. According to transmission line theory, the input impedance can be expressed as

$Z_{\text {short }}=Z_{0} \tanh (\gamma l)$

where

$\gamma=\sqrt{\frac{R+j \omega L}{G+j \omega C}}$

is the propagation constant, $Z_{0}$ is the characteristic impedance of the section of Chapter 3. Time Domain Calibration Standards 
offset transmission line and $l$ is the offset length.

\subsubsection{Coaxial Open Circuit Standard}

The discontinuity open can be modeled as a resistor and capacitor in parallel. For the end closed open circuit, the open discontinuity can be approximately modeled as a capacitor, as shown in Fig. 3.1.2. The derivation of this capacitance value is given in Appendix B. According to transmission line theory, the input impedance can be expressed as

$Z_{\text {open }}=Z_{0} \frac{Z_{L}+Z_{0} \tanh (\gamma l)}{Z_{0}+Z_{L} \tanh (\gamma l)}$

where

$Z_{L}=\frac{1}{j \omega C_{\text {open }}}$

$C_{\text {open }}$ is the equivalent capacitance for the open discontinuity. Due to lack of design information, the $C_{\text {open }}$ model provided by the manufacture,

$\mathrm{C}_{\text {open }}=C_{0}+C_{1} f+C_{2} f^{2}+C_{s} f^{s}$

is actually used to calculate the $Z_{\text {open }}$ [17]. In equation (3.5.5), $C_{0}=43.45 \times 10^{-15} \mathrm{~F}$, $C_{t}=818.7 \times 10^{-27} \mathrm{~F} / \mathrm{H}_{Z}, C_{2}=-48.93 \times 10^{-96} \mathrm{~F} / \mathrm{H}_{Z}{ }^{2}$, and $C_{9}=1.247 \times 10^{-45} \mathrm{~F} / \mathrm{H}_{Z}{ }^{s} . \quad f$ is the operating frequency in Hertz. 


\subsection{ERROR SOURCES}

For coaxial calibration standards, there are several error sources: environmental conditions, mechanical tolerances and intrinsic material properties. We discuss their effects and how to reduce their effects in this section.

\subsubsection{Environmental Conditions}

Environmental conditions are critical to the coaxial standards because they will change the geometrical size of a coaxial line, permittivity of the air and conductivity of the metal. In order to reduce this error, the so called "standard conditions are accepted internationally. The standard conditions are $23^{\circ} \mathrm{C}$ ambient temperature, 50 percent relative humidity and an atmospheric pressure of $760 \mathrm{~mm}$ of mercury.

\subsubsection{Mechanical Tolerances}

As shown in section 3.3, the characteristic impedance is related to the geometrical dimensions of a coaxial transmission line. In practice, however, the mechanical tolerances on the air line dimensions, the eccentricity and the circularity of the air line conductors are not avoidable. This will limit the accuracy of the line standards.

A simple sensitivity analysis is performed to show the relationship of the reflection coefficient $\Gamma$ versus the dimension of inner conductor. The results obtained are 
shown in Fig. 3.6.1. From this figure, we can choose the $\Gamma$ values which is relatively insensitive to the inner conductor's dimension. In this way, the effects of the dimension uncertainties can be reduced. 
Table 3.4.1 Computed values of step capacitance in $\mathrm{fF} / \mathrm{cm}$

$\begin{array}{lllllll}\alpha & \tau=1 & 1.5 & 3 & 5 & 10 & \infty \\ 0.1 & 108.20 & 108.56 & 110.31 & 112.05 & 114.56 & 134.89 \\ 0.2 & 69.700 & 70.024 & 71.663 & 73.320 & 75.736 & 95.659 \\ 0.3 & 47.797 & 48.083 & 49.562 & 51.089 & 53.349 & 72.607 \\ 0.4 & 32.931 & 33.171 & 34.452 & 35.808 & 37.859 & 56.171 \\ 0.5 & 22.121 & 22.311 & 23.363 & 24.514 & 26.302 & 43.358 \\ 0.6 & 14.057 & 14.196 & 14.999 & 15.913 & 17.387 & 32.832 \\ 0.7 & 8.0688 & 8.1592 & 8.7063 & 9.3604 & 10.469 & 23.869 \\ 0.8 & 3.7965 & 3.8436 & 4.1462 & 4.5311 & 5.2314 & 16.009 \\ 0.9 & 1.0784 & 1.0926 & 1.1927 & 1.3303 & 1.6095 & 8.8579 \\ 1.0 & 0.0 & 0.0 & 0.0 & 0.0 & 0.0 & 0.0\end{array}$

Note: when multiplied by the circumference of the outer conductor, these figures yield the value of step capacitance in femtofarads. 


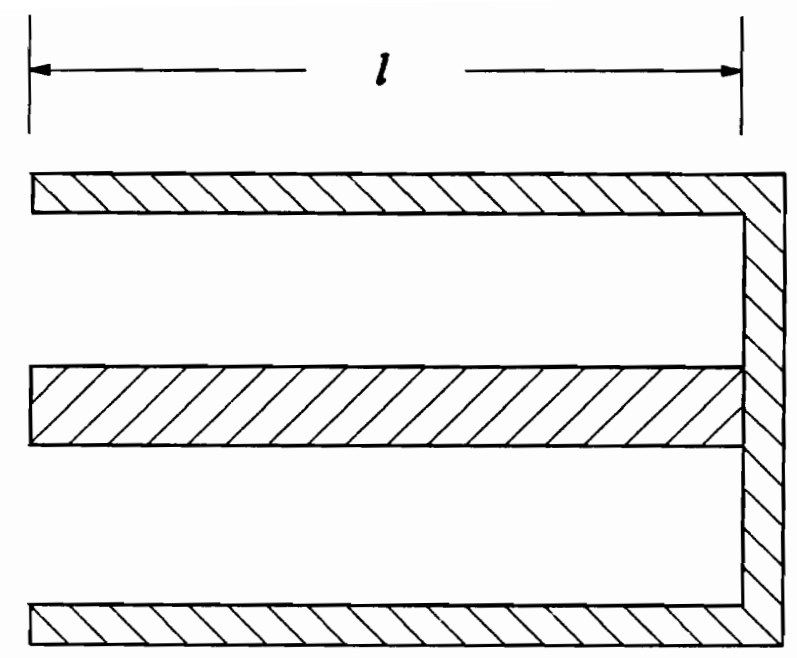

(a)

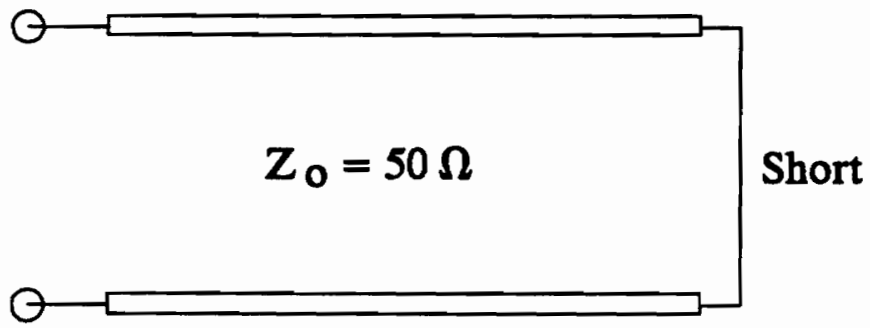

(b)

Figure 3.1.1 Offset short circuit standard (a) and its equivalent circuit (b). 


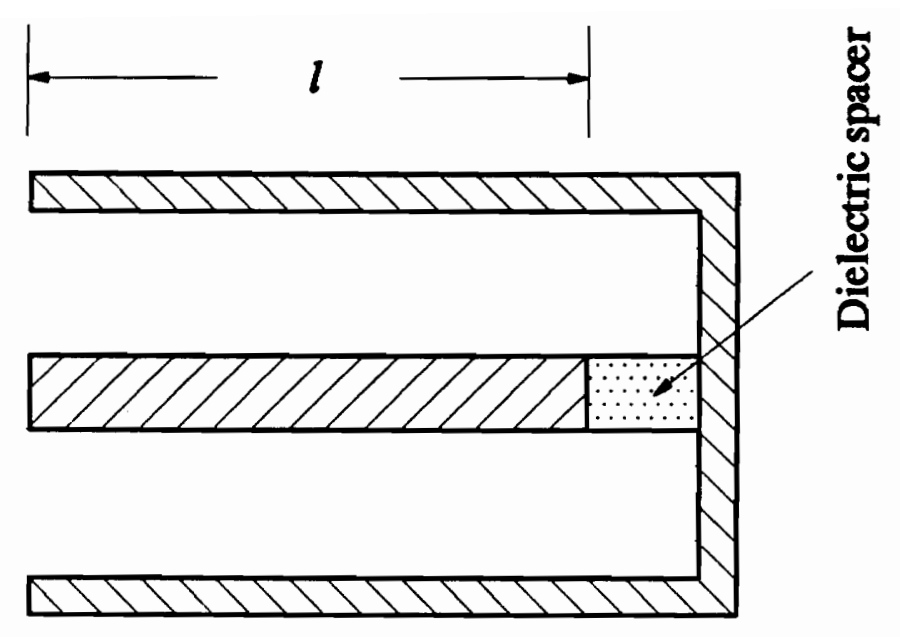

(a)

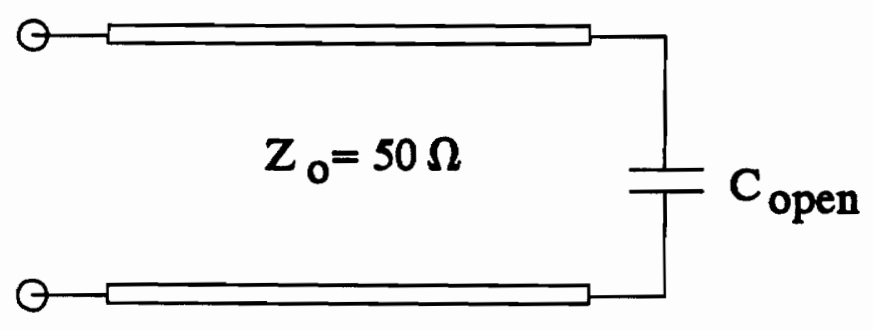

(b)

Figure 3.1.2 Offset open circuit standard (a) and its equivalent circuit (b). 


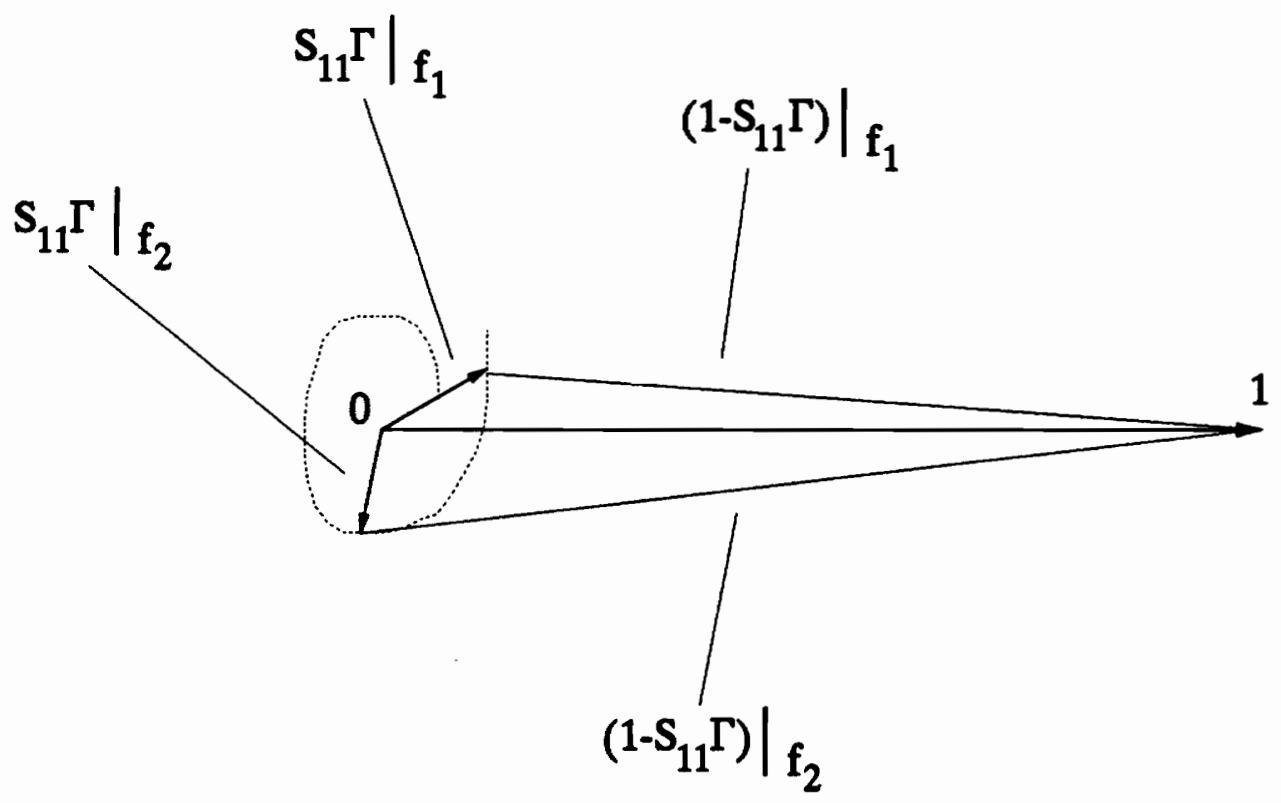

Figure 3.2.1 Variation of (1-S11 $\Gamma)$ with frequency. 

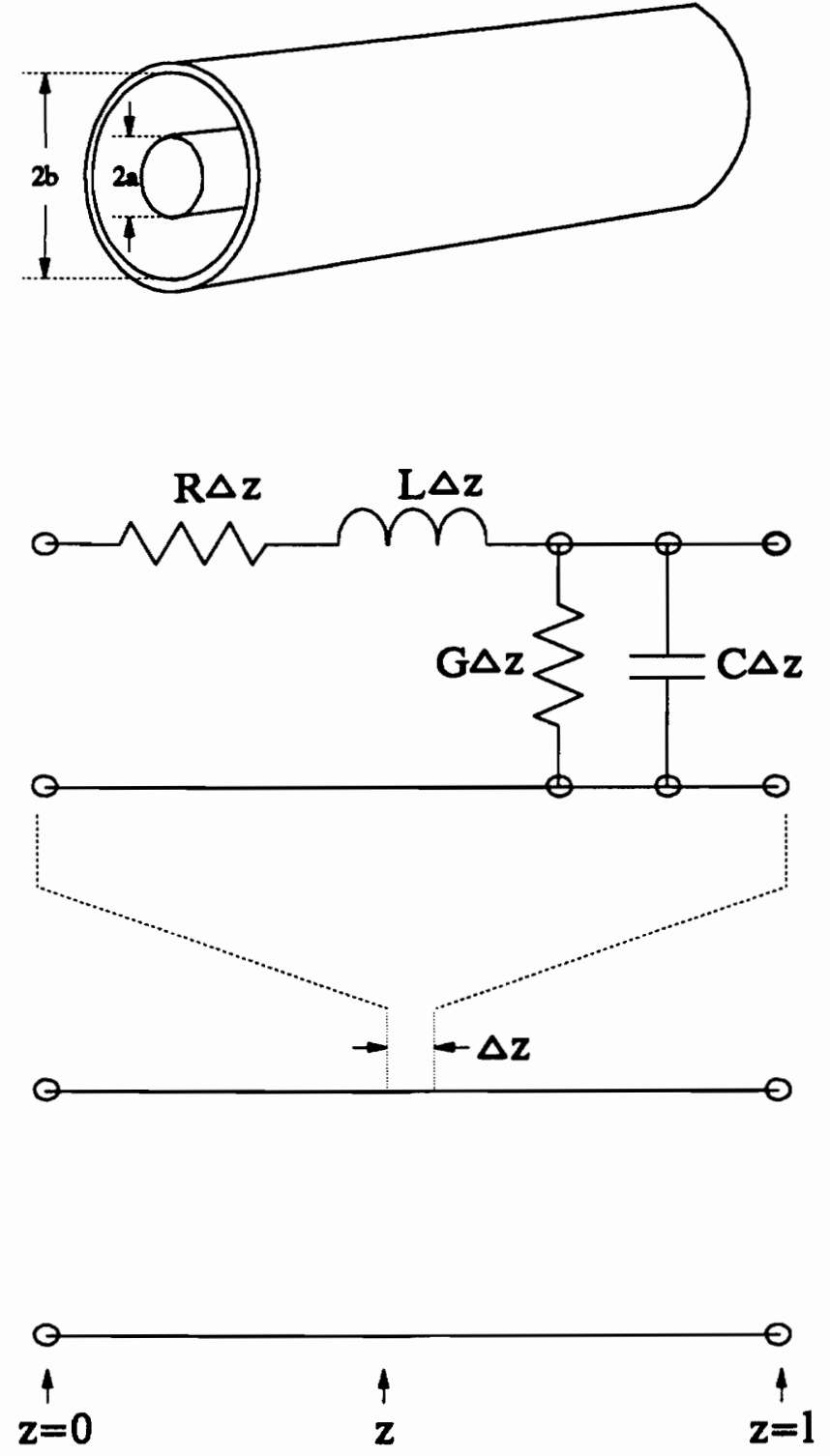

Figure 3.3.1 Coaxial transmission line and its equivalent circuit. 


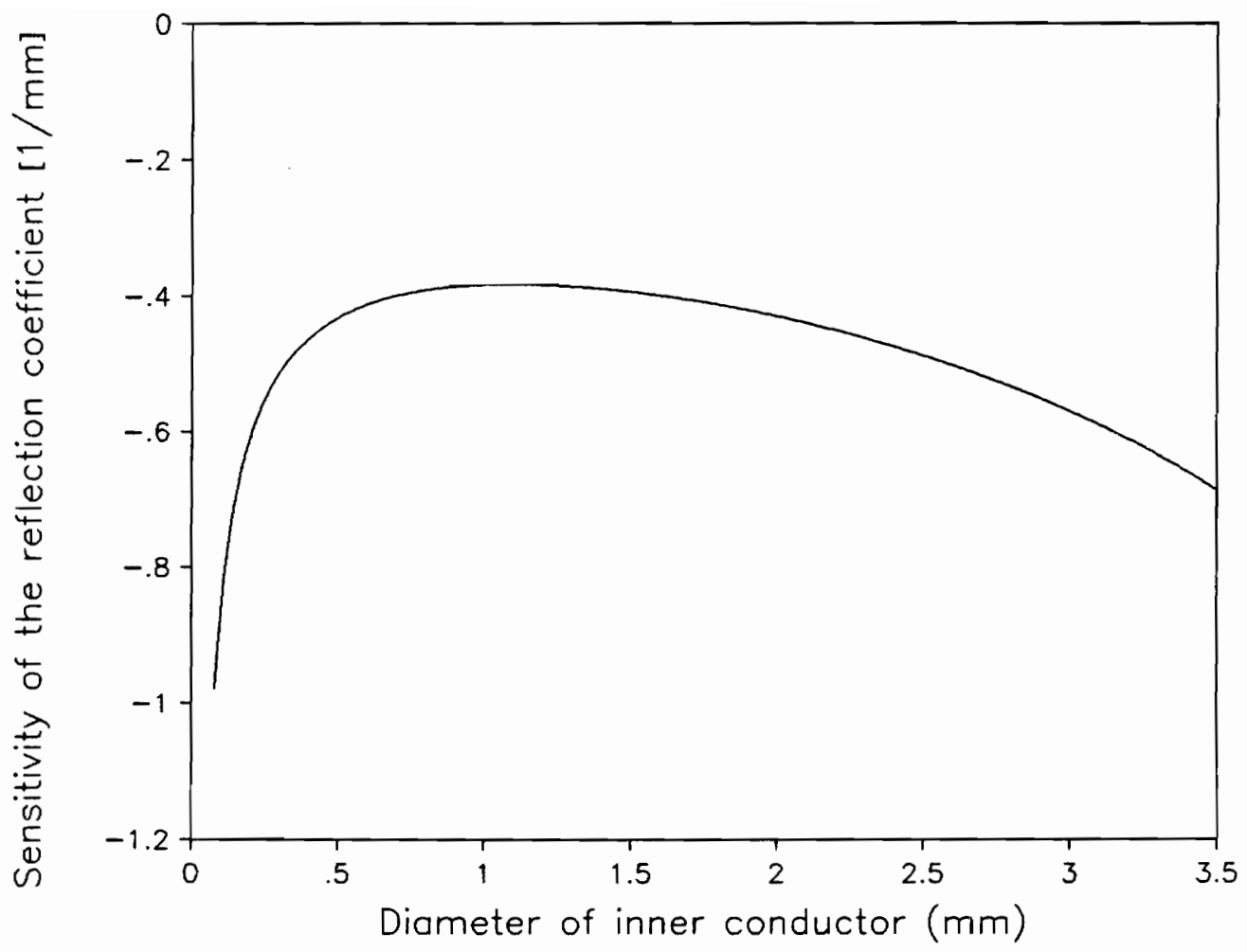

Figure 3.6.1 The sensitivity of the reflection coefficient $\Gamma$ versus dimension of inner conductor for a $3.5 \mathrm{~mm}$ coaxial air filled transmission line. 


\section{CHAPTER 4}

\section{EXPERIMENTAL DATA PROCESSING}

\subsection{INTRODUCTION}

In this chapter, we discuss the nature of the measurement data as well as signal processing techniques required to extract various information from these data. Signal averaging, Fourier transformation, and zero padding are among the signal processing techniques used and are discussed here. Errors such as windowing and truncation which affect the results of the processed information are also identified and discussed.

First, let us identify with the nature of the experimental data obtained from the TDNA measurement, namely, the time domain waveform. The acquired data, $v_{s d}\left(t_{k}\right)$, are in the form of a real voltage waveform which is available in a discrete form. Such a waveform can be expressed as:

$v_{s d}\left(t_{k}\right)=v(t) * h(t) \cdot w_{\Pi}(t) \cdot \delta\left(t-t_{k}\right)+n_{k}, \quad$ for $k=0, \ldots, N$

where : $\quad v(t)$ is the equivalent continuous real waveform; 
$h(t)$ is the impulse response of the measurement system;

$w_{\Pi}(t)$ is the window function, a weighted filter applied to the waveform (unity for $0 \leq t \leq T$, zero elsewhere);

$\delta\left(t-t_{k}\right)$ is the Dirac delta function (unity at $t=t_{k}$, zero elsewhere);

$n_{k}$ is the random measurement noise at $t_{k}$ instance;

$t_{0}=0$ and $t_{N}=T$, the duration of the waveform epoch.

The main task of processing the experimental data is to extract useful information from equation (4.1.1).

\subsection{SIGNAL AVERAGING}

Experimental data are not extremely precise and repetition is likely to produce a slightly different answer for each measurement conducted under the same condition. In order to overcome this error, the averaging method is introduced. The statistical analysis is used to support the analysis of the averaging processing [18].

In the waveform of equation (4.1.1), there is a random noise component, $n_{k}$, in the measurement data. This noise component determines the signal to noise ratio $(S / N)$. In order to reduce the measurement noise, the signal averaging method can be used. As known, the measurement noise has two features. First, the mean value of the noise is zero, implying no bias in the system. Second, the variance $\sigma_{n}^{2}$ of the noise over the period $T$ is constant. This interprets that the average noise 
power does not change with time. Under these conditions, the $S / N$ of the $k$ th sample is of the form,

$(S / N)_{k}=\frac{v(t) * h(t) \cdot w_{\Pi}(t) \cdot \delta\left(t-t_{k}\right)}{\sigma_{n}}$

After $M$ repetitions of the signal, the summation value of the $k$ th sample is,

$$
\begin{aligned}
\sum_{i=1}^{M} v_{s d}\left(t_{i k}\right) & =\sum_{i=1}^{M} v(t) * h(t) \cdot w_{\Pi}(t) \cdot \delta\left(t-t_{i k}\right)+\sum_{i=1}^{M} n_{i k} \\
& =M \cdot v(t) * h(t) \cdot w_{\Pi}(t) \cdot \delta\left(t-t_{i k}\right)+\sum_{i=1}^{M} n_{i k}
\end{aligned}
$$

For the signal samples, one expects a summation. But, as the variance of all the $k$ th noise samples are considered equal, the total noise power should be the sum of the noise power of each noise sample and equal to $M \sigma_{n}{ }^{2}$. Then, the total root mean square (r.m.s.) noise value is $\sqrt{M} \sigma_{n}$ and the signal/noise ratio after $M$ repetitions will be:

$(S / N)=\frac{M \cdot v(t) * h(t) \cdot w_{\Pi}(t) \cdot \delta\left(t-t_{i k}\right)}{\sqrt{M} \sigma_{n}}=\sqrt{M}(S / N)_{k}$

Thus the summation of $M$ repetitions of the noisy signal results in a $\sqrt{M}$ improvement in the signal to noise ratio. Theoretically, one can increase the signal to noise ratio through further increase in $M$. In practice, however, due to the time axis and voltage drifting, increasing $M$ is not proportional to the improvement of the signal to noise ratio. 


\subsection{FOURIER TRANSFORM}

The measurements are performed in the time domain and the model parameters are evaluated in the frequency domain. Transforming the time domain data into frequency domain is inevitable. The connection between the time domain and the frequency domain is the Fourier transform [19]. The Fourier transform pair for continuous signals are defined as

$$
\begin{aligned}
& V(f)=\int_{-\infty}^{\infty} v(t) e^{-j 2 \pi f t} d t \quad \text { and } \\
& v(t)=\int_{-\infty}^{\infty} V(f) e^{j 2 \pi f t} d f
\end{aligned}
$$

where $v(t)$ is the time domain waveform, and $V(f)$ is its corresponding frequency domain spectrum or representation.

From a time domain system, the obtained waveform is a set of discrete time domain data $v_{s d}\left(t_{k}\right)$. For the set of discrete data, the discrete Fourier transform is needed and is defined as follows.

$$
\begin{aligned}
& V\left(f_{n}\right)=\frac{1}{T} \sum_{k=0}^{N-1} v_{s d}\left(t_{k}\right) e^{-j 2 \pi f_{n}{ }^{i} k} \Delta t_{k} \\
& v_{s d}\left(t_{k}\right)=\sum_{n=0}^{N-1} V_{n}\left(f_{n}\right) e^{j 2 \pi f_{n} i_{k}}
\end{aligned}
$$

If an equal sampling interval $\Delta t=\Delta t_{k}$ is assumed, then, $t_{k}=k \Delta t$ and $\mathrm{T}=\mathrm{N} \Delta t$. Assuming an equal frequency interval $\Delta f=\frac{1}{T}$, then, $f_{n}=n \Delta f$. Equations (4.3.3) 
and (4.3.4) can be rewritten as,

$$
\begin{aligned}
& V(n \Delta f)=\frac{1}{N} \sum_{k=0}^{N-1} v_{s d}(k \Delta t) e^{-j 2 \pi k n / N}, \\
& v_{s d}(k \Delta t)=\sum_{n=0}^{N-1} V(n \Delta f) e^{j 2 \pi k n / N} .
\end{aligned}
$$

The forms of equations (4.3.5) and (4.3.6) represent a suitable convenient form for explaining the concept of the fast Fourier transform.

\subsection{FAST FOURIER TRANSFORM (FFT)}

From equation (4.3.5), in order to make the derivation more concise, let $W=e^{-j 2 \pi / N}, X(n)=V(n \Delta f)$ and $x(k)=v_{s d}(k \Delta t) / N$. Then, one can obtain,

$$
\begin{aligned}
& X(n)=\sum_{k=0}^{N-1} x(k) W^{n k} \\
& x=0,1, \ldots, N-1 \\
& x(k)=\sum_{n=0}^{N-1} X(n) W^{-n k}
\end{aligned}
$$

In this respect, the number of the sample points $N, N=\mathscr{2}^{\gamma}$, and $\gamma$ is a positive integer. Then, one can express $\mathrm{n}$ and $\mathrm{k}$ in terms of the index $\gamma$, as a binaryweighted series:

$n=\mathscr{2}^{\gamma-1} n_{y-1}+\mathscr{2}^{\gamma-1} n_{y-1}+\ldots+n_{0}$ 
$k=\mathscr{2}^{\gamma-1} k_{y-1}+2^{\gamma-1} k_{y-1}+\ldots+k_{0}$

Using these expressions, equation (4.4.1) can be written as

$X\left(n_{y-1}, n_{y-2}, \ldots, n_{0}\right)=\sum_{k_{0}=0}^{1} \sum_{k_{1}=0}^{1} \ldots \sum_{k_{\gamma-1}=0}^{1} x\left(k_{y-1}, k_{y-2}, \ldots, k_{0}\right) W^{P}$

where

$P=\left(\mathscr{2}^{\gamma-1} n_{y-1}+2^{\gamma-1} n_{y-1}+\ldots+n_{0}\right)\left(2^{\gamma-1} k_{y-1}+2^{\gamma-1} k_{y-1}+\ldots+k_{0}\right)$

Since $W^{a+b}=W^{a} \cdot W^{b}, W^{P}$ can be written as

$$
\begin{aligned}
W^{P}= & \left.W^{\left(2^{\gamma-1} n_{y-1}+2^{\gamma-1} n_{y-1}+\cdots+n_{0}\right) \cdot\left(2^{\gamma-1} k_{y-1}\right.}\right) \\
& \left.\cdot W^{\left(2^{\gamma-1} n_{y-1}+2^{\gamma-1} n_{y-1}+\ldots+n_{0}\right) \cdot\left(2^{\gamma-2} k_{y-2}\right.}\right) \\
& \left.\cdots W^{\left(2^{\gamma-1} n_{y-1}+2^{\gamma-1} n_{y-1}+\ldots+n_{0}\right) \cdot\left(2^{0} k_{0}\right.}\right)
\end{aligned}
$$

Now, let us examine the first term in the above expression

$$
\begin{aligned}
& W^{\left(2^{\gamma-1} n_{y-1}+2^{\gamma-1} n_{y-1}+\ldots+n_{0}\right) \cdot\left(2^{\gamma-1} k_{y-1}\right)} \\
= & \left.W^{2^{\gamma}\left(2^{\gamma-2} n_{y-1} k_{y-1}\right)} \cdot W^{2^{\gamma}\left(2^{\gamma-s_{n}}\right.} 2_{y-2^{k}-1}\right) \cdots W^{2^{\gamma-1}\left(n_{0} k_{y-1}\right)} \\
= & W^{2^{\gamma-1}\left(n_{0} k_{y-1}\right)}
\end{aligned}
$$

The equality, $W^{2^{\gamma}}=W^{N}=\left[e^{-j 2 \pi / N}\right]^{N}=1$, was used to simplify the expression. Similarly, the second term can be reduced as, 
$W^{\left(2^{\gamma-1} n_{y-1}+2^{\gamma-1} n_{y-1}+\ldots+n_{0}\right) \cdot\left(2^{\gamma-2 k_{y-2}}\right)}=W^{2^{\gamma-2}\left(2 n_{1}+n_{0}\right) k_{y-2}}$

Using the same procedure, we perform the desired reduction. It is clear that in this procedure, there will be an additional term that can not be simplified each time using the condition $\mathrm{W}^{2^{\gamma}}=1$. For the last term in equation (4.4.6), no further simplification is possible. Utilizing the obtained results, equation (4.4.4) can be written as

$$
\begin{aligned}
& X\left(n_{y-1}, n_{y-2}, \ldots, n_{0}\right)=\sum_{k_{0}=0}^{1} \sum_{k_{1}=0}^{1} \ldots \sum_{k_{\gamma}=0}^{1} x\left(k_{y-1}, k_{y-2}, \ldots, k_{0}\right) W^{2^{\gamma-1}\left(n_{0} k_{y-1}\right)} . \\
& W^{2^{\gamma-2}\left(2 n_{1}+n_{0}\right) k_{y-2}} \ldots W^{\left(2^{\gamma-1} n_{y-1}+2^{\gamma-1} n_{y-1}+\ldots+n_{0}\right) \cdot\left(k_{0}\right)} \\
& x_{1}\left(n_{0}, k_{y-2}, \ldots, k_{0}\right)=\sum_{k_{\gamma-1}=0}^{1} x\left(k_{y-1}, k_{y-2}, \ldots, k_{0}\right) W^{2^{\gamma-1}\left(n_{0} k_{y-1}\right)} \\
& x_{2}\left(n_{0}, n_{1}, k_{y-s}, \ldots, k_{0}\right)=\sum_{k_{\gamma}-2=0}^{1} x_{1}\left(n_{0}, k_{y-2}, \ldots, k_{0}\right) W^{2^{\gamma-2}\left(2 n_{1}+n_{0}\right) k_{y-2}} \\
& \quad \cdot \\
& x_{\gamma}\left(n_{0}, n_{1}, \ldots, n_{\gamma-1}\right)=\sum_{k_{0}=0}^{1} x_{\gamma-1}\left(n_{0}, n_{1}, \ldots, n_{y-2}, k_{0}\right) W^{\left(2^{\gamma-1} n_{y-1}+2^{\gamma-1} n_{y-1}+\ldots+n_{0}\right) k_{0}} \\
& X\left(n_{y-1}, n_{y-2}, \ldots, n_{0}\right)=x_{\gamma}\left(n_{0}, n_{1}, \ldots, n_{\gamma-1}\right)
\end{aligned}
$$

The last equation shows that, in order to find $X$, the order of the bits in the binary representation of $n$ must be reversed to obtain the correct index. This set of recursive equations represented the original Cooley-Tukey FFT algorithm. As 
known, if one directly calculates equation (4.4.1), $N^{2}$ complex multiplications are needed. Using equations (4.4.10), we have $\gamma$ summing equations. Each equation represents $N$ equations. Each equation contains two complex multiplications and one addition. But, the first multiplication of the two actually times one because the first multiplication always time the factor of the form $W^{a k_{\gamma-i}}$ while $k_{\gamma-i}=0$. Thus, the total number of multiplication operations is only $N_{\gamma}$. The algorithm, however, requires that the number of the data should be the integer power of 2 . In the case the number of measurement data is not the integer power of 2 , the zero padding method is often used to overcome this problem. This method is the topic of the following section.

\subsection{ZERO PADDING}

One can keep the same time domain resolution to increase the frequency domain resolution through increasing the sampling points $N$ using the FFT. The maximum points of output data for a measurement system is usually limited. In order to further increase $N$, one of the most common way for impulsive waveforms is the zero padding, simply adding zero values to the original data.

The process consists of adding $N$ zero values $x(N), \ldots, x(2 N-1)$ to the original data samples $x(0), \ldots, x(N-1)$. The FFT of the zero-padded $2 N$-point data sequence is of the form,

$X(k)=T \sum_{n=0}^{2 N-1} x(n) e^{-j 2 \pi n k / 2 N}=T \sum_{n=0}^{N-1} x(n) e^{-j 2 \pi n k / 2 N}$

Chapter 4. Experimental Data Processing 
where the range on the right hand summation has been adjusted to reflect the zero samples. Let $k=2 l$, so that

$$
X(l)=T \sum_{n=0}^{N-1} x(n) e^{-j 2 \pi n / / 2 N} \quad \text { for } l=0,1, \ldots, N-1
$$

$X(l)$ represents the even values of $X(k)$. The $2 N$-point FFT, therefore, reduces to that of the N-point FFT at the even index values. The odd values of $k$ represent the interpolated FFT values between the original N-point FFT values.

As more zeros are padded to the original N-point sequence, even more interpolation is obtained. In the limit of infinite zero padding, the FFT may be viewed as taking the FFT of an $N$-point windowed data sequence,

$$
X(f)=T \sum_{n=0}^{N-1} x(n) e^{-j 2 \pi f n T}
$$

Actually, the zero padding provides an interpolated transform to improve the frequency resolution. The accuracy of estimating the frequency of spectral peaks is also enhanced with zero padding.

\subsection{WAVEFORM TRUNCATION}

A signal cannot simultaneously be both time-limited and bandlimited [20]. Since the measurement system usually is bandlimited, the time domain truncation is inevitable. Let us analyze the effects of this truncation. 
When one use a measurement system to acquire physical signals, one can only get a time-limited waveform irrespect of a long time window. Assuming a time domain waveform $x(t)$ and a time window $w_{\Pi 1}$, then, the measured waveform $y(t)$ is of the form,

$y(t)=x(t) \cdot w_{\Pi}$

If $x(t)$ is time-limited within the window, the window $w_{\Pi}$ will not cause any error. For $x(t)$ not time-limited, the error obtained in frequency domain needs to be evaluated. Applying the Fourier transform to $x(t)$ and $y(t)$, and taking the spectrum difference, one can have

$\epsilon(f)=\int_{-\infty}^{\infty} x(t) e^{-j 2 \pi f t} d t-\int_{-T / 2}^{T / 2} x(t) e^{-j 2 \pi f t} d t$

From this equation, it is clear that if we want to reduce the frequency domain spectrum error, the only way is to increase the time window. This means that in order to avoid the spectrum error, theoretically, we have to extend the time window to include all the time domain information, an unrealistic practice. However, a time domain waveform can still be characterized by an interval of $T$ in which most of its temporal energy is concentrated. If we adjust the time window to include most of the signal energy inside the window, we can minimize the spectrum error although we can not include all the time domain information.

On the other hand, applying Fourier transform to equation (4.6.1), we get 
$Y(f)=X(f) * \sin c(f)$.

The time domain window $w_{\Pi 1}$ causes aliasing error which extends the spectrum to infinity even if $x(t)$ is a band-limited signal.

Since the $\operatorname{sinc}(f)$ is not a positive function, the convolution will cause the oscillation in the spectrum. In order to avoid this, there are many window shapes with minimum sidelobes and maximum sidelobe decay can be selected. The Nuttal window is worthy to be mentioned because of its superior performance. The definition of the Nuttal window is

$w=\sum_{r=0}^{M} a_{r} \cos \left(2 \pi r \frac{k-(N-1) / 2}{N-1}\right)$

For a $M=3$ four-term window, $a_{0}=0.3635819, a_{1}=0.4891775, a_{2}=0.1365995$ and $a_{4}=0.0106411$. The highest sidelobe of this window is only $-98 \mathrm{~dB}$, in contrast, the highest sidelobe of a rectangular window can reach $-19.9 \mathrm{~dB}$. Using this window instead of the rectangular window (caused by truncation), the truncation error can be effectively reduced. 


\section{CHAPTER 5}

\section{ERROR ANALYSIS}

\subsection{INTRODUCTION}

Error is the deviation from the true value of the measured quantity. It is not avoidable in any experimental measurement. Some error will always be present irrespect of how carefully the measurements are taken, and how accurate the equipment used. A measurement is useful only when the size and type of the anticipated error is known. A major skill in conducting the measurements is the ability to interpret results in terms of possible errors. In a TDNA system, errors can be classified into two major types: systematic errors and random errors.

Systematic errors are the type of errors which remain constant with repeated measurements. These errors do not change (or change very slowly) with time and can be measured and compensated for. Typical systematic errors are modeling errors, offset errors, scale errors and system nonlinearities. Random errors are undeterministic errors whose magnitude and polarity fluctuate in an unpredictable manner. Typical random errors are quantization errors, roundoff errors and time and signal channel noise. 
Whether an error is considered as systematic error or random error, it also depends on actual conditions. For example, in the TDNA calibration, the environmental conditions, standards, and connectors can cause measurement errors. These errors may affect the calibration in a random manner. But once calibrated, measurements will be affected only by the same "systematic error" in the calibration.

Since most of these errors are extensively studied [10],[11], only the major errors for our TDNA system, such as the quantization error, modeling and de-embedding errors are discussed in this chapter.

\subsection{QUANTIZATION ERROR}

Quatization error is one of the major errors in a TDNA system. The error directly determines the accuracy of acquired TDNA waveforms which are the base of time domain analyses. The quantization error comes from using an $A / D$ convertor to convert a continuous analog signal into a digital number. This process can only be an approximation since the analog signal can assume an infinite number of states while the number of bits in a digital representation is limited. The difference between the two represents the quantization error.

In Fig. 5.2.1, the input to the A/D convertor is illustrated in staircase form and this is the practical format in a TDNA system. In broad terms, quantization is a non-linear operation that is carried out whenever a physical quantity is represented 
numerically. The resultant numerical value is given as an integer corresponding to the nearest whole number of units. For this reason, it is convenient to think of the process in statistical terms, and to consider the quantized series as representing the probability density distribution of the input series. The transfer characteristics of a quantizer can be represented as in Fig. 5.2.2. An input value lying between the midpoint values of two consecutive unit values will produce an output value at the level corresponding to the higher of the two.

Assuming a physical signal $x$, a digitized number $x_{q}$ is obtained from an A/D convertor. The relation between these two numbers is of the form,

$x_{q}=x+x_{n}$

where $x_{n}$ is the quantization noise. This noise represents a quantization error, shown in Fig. 5.2.3 as a function of the quantizer input. The quantization error is clearly related to the least value of quantization $q$, and can be given as the ratio of the magnitude of the exact sample number $A$, and the approximate number expressed as a power of 2 ,

$\epsilon= \pm \frac{A}{2} \frac{1}{2^{N}}= \pm \frac{q}{2}$

To assess the value of this error, let $p(x)$ be the quantization error probability density function defined by

$$
\begin{aligned}
p(x) & =1, & & \text { for }-0.50 \geq x \geq+0.50 \\
& =0, & & \text { otherwise. }
\end{aligned}
$$


The variance for this error is of the form,

$$
\sigma^{2}=q^{2} \int_{-\infty}^{\infty}(x-\bar{x})^{2} p(x) d x
$$

and since the mean value must be zero,

$$
\sigma^{2}=q^{2} \int_{-0.5}^{0.5} x^{2} d x=q^{2}\left(\frac{x^{3}}{3}\right)_{-0.5}^{0.5}=\frac{q^{2}}{12}
$$

giving a standard deviation for a single unit of quantization of $\sigma_{x}=1 / \sqrt{12}=0.29$ level units. This gives a value for the quantization noise which is added to the desired signal. As an example, the HP 45120 system has an A/D converter with 11 effective binary bits. This means that we can quantize a signal to 2048 level units. Then for a noise free input signal, the signal/noise ratio at the output of the A/D converter will be $2048 / 0.29 \simeq 7000$ or about $76 \mathrm{~dB}$. This is adequate to represent the input signal and has a negligible effect comparing to the whole system dynamic range of $40 \mathrm{~dB}$.

\subsection{TDR MODELING ERRORS}

For the obtained TDR model, the model parameters relate to the standards and the measured responses of the standards are as follows:

$$
\mathrm{S}_{11}=\frac{W_{r 1}\left(\Gamma_{3}-\Gamma_{2}\right)+W_{r 2}\left(\Gamma_{1}-\Gamma_{3}\right)+W_{r 3}\left(\Gamma_{2}-\Gamma_{1}\right)}{W_{r 1} \Gamma_{1}\left(\Gamma_{3}-\bar{\Gamma}_{2}\right)+W_{r 2} \Gamma_{2}\left(\Gamma_{1}-\Gamma_{3}\right)+W_{r g} \Gamma_{3}\left(\Gamma_{2}-\Gamma_{1}\right)},
$$


$S_{g r}=\frac{\left(W_{r 1} \Gamma_{2}-W_{r 2} \Gamma_{1}\right)+\Gamma_{1} \Gamma_{2} S_{11}\left(W_{r 2}-W_{r 1}\right)}{E_{g}\left(\Gamma_{2}-\Gamma_{1}\right)}$,

and

$\mathrm{S}_{g 1} S_{t r}=\left(\frac{1}{\Gamma_{1}}-S_{11}\right)\left(\frac{W_{r 1}}{E_{g}}-S_{g r}\right)$

In order to analyze the modeling errors, one need to analyze the sensitivities of the model parameters to the standards and the measurement results [21]. First, we take the derivatives of the model parameters to the $\Gamma$ s and $W s$, respectively. From $S_{11}$ expression, we can get,

$\frac{\partial S_{11}}{\partial \Gamma_{1}}=\frac{\left(\Gamma_{2}-\Gamma_{s}\right)^{2}\left(W_{r 1} W_{r 2}+W_{r 1} W_{r s}-W_{r 2} W_{r s}-W_{r 1}^{2}\right)}{\left[W_{r 1} \Gamma_{1}\left(\Gamma_{s}-\Gamma_{2}\right)+W_{r 2} \Gamma_{2}\left(\Gamma_{1}-\Gamma_{s}\right)+W_{r s} \Gamma_{s}\left(\Gamma_{2}-\Gamma_{1}\right)\right]^{2}}$,

$\frac{\partial S_{11}}{\partial \Gamma_{2}}=\frac{\left(\Gamma_{s}-\Gamma_{1}\right)^{2}\left(W_{r 1} W_{r 2}+W_{r 2} W_{r s}-W_{r 1} W_{r g}-W_{r 2}^{2}\right)}{\left[W_{r 1} \Gamma_{1}\left(\Gamma_{s}-\Gamma_{2}\right)+W_{r 2} \Gamma_{2}\left(\Gamma_{1}-\Gamma_{s}\right)+W_{r s} \Gamma_{s}\left(\Gamma_{2}-\Gamma_{1}\right)\right]^{2}}$,

$\frac{\partial S_{11}}{\partial \Gamma_{s}}=\frac{\left(\Gamma_{1}-\Gamma_{2}\right)^{2}\left(W_{r 1} W_{r s}+W_{r 2} W_{r s}-W_{r 1} W_{r 2}-W_{r s}{ }^{2}\right)}{\left[W_{r 1} \Gamma_{1}\left(\Gamma_{s}-\Gamma_{2}\right)+W_{r 2} \Gamma_{2}\left(\Gamma_{1}-\Gamma_{s}\right)+W_{r s} \Gamma_{s}\left(\Gamma_{2}-\Gamma_{1}\right)\right]^{2}}$,

$\frac{\partial S_{11}}{\partial W_{r 1}}=\frac{\left(\Gamma_{s}-\Gamma_{2}\right)\left(\Gamma_{1}-\Gamma_{s}\right)\left(\Gamma_{2}-\Gamma_{1}\right)\left(W_{r 2}-W_{r s}\right)}{\left[W_{r 1} \Gamma_{1}\left(\Gamma_{3}-\Gamma_{2}\right)+W_{r 2} \Gamma_{2}\left(\Gamma_{1}-\Gamma_{s}\right)+W_{r s} \Gamma_{s}\left(\Gamma_{2}-\Gamma_{1}\right)\right]^{2}}$,

$\frac{\partial S_{11}}{\partial W_{r 2}}=\frac{\left(\Gamma_{s}-\Gamma_{2}\right)\left(\Gamma_{1}-\Gamma_{s}\right)\left(\Gamma_{2}-\Gamma_{1}\right)\left(W_{r g}-W_{r 1}\right)}{\left[W_{r 1} \Gamma_{1}\left(\Gamma_{g}-\Gamma_{2}\right)+W_{r 2} \Gamma_{2}\left(\Gamma_{1}-\Gamma_{s}\right)+W_{r g} \Gamma_{s}\left(\Gamma_{2}-\Gamma_{1}\right)\right]^{2}}$,

and

$\frac{\partial S_{11}}{\partial W_{r s}}=\frac{\left(\Gamma_{s}-\Gamma_{2}\right)\left(\Gamma_{1}-\Gamma_{s}\right)\left(\Gamma_{2}-\Gamma_{1}\right)\left(W_{r 1}-W_{r 2}\right)}{\left[W_{r 1} \Gamma_{1}\left(\Gamma_{s}-\Gamma_{2}\right)+W_{r 2} \Gamma_{2}\left(\Gamma_{1}-\Gamma_{s}\right)+W_{r s} \Gamma_{s}\left(\Gamma_{2}-\Gamma_{1}\right)\right]^{2}}$.

Then, the possible error in determining the $S_{11}$ parameter is of the form, 


$$
\begin{aligned}
\left|\Delta S_{11}\right| & =\left|\frac{\partial S_{11}}{\partial \Gamma_{1}} \Delta \Gamma_{1}\right|+\left|\frac{\partial S_{11}}{\partial \Gamma_{2}} \Delta \Gamma_{2}\right|+\left|\frac{\partial S_{11}}{\partial \Gamma_{s}} \Delta \Gamma_{s}\right|+\left|\frac{\partial S_{11}}{\partial W_{r 1}} \Delta W_{r 1}\right|+\left|\frac{\partial S_{11}}{\partial W_{r 2}} \Delta W_{r 2}\right| \\
& +\left|\frac{\partial S_{11}}{\partial W_{r s}} \Delta W_{r s}\right| \cdot
\end{aligned}
$$

From $S_{g r}$ expression, one can get,

$$
\begin{aligned}
\frac{\partial S_{g r}}{\partial \Gamma_{1}}= & \frac{\Gamma_{2}\left(W_{r 1}-W_{r 2}\right)\left[1-\Gamma_{2} S_{11}-\Gamma_{1}\left(\Gamma_{2}-\Gamma_{1}\right) \frac{\partial S_{11}}{\partial \Gamma_{1}}\right]}{E_{g}\left(\Gamma_{2}-\Gamma_{1}\right)^{2}} \\
\frac{\partial S_{g r}}{\partial \Gamma_{2}} & =\frac{\Gamma_{1}\left(W_{r 2}-W_{r 1}\right)\left[1-\Gamma_{1} S_{11}+\Gamma_{2}\left(\Gamma_{2}-\Gamma_{1}\right) \frac{\partial S_{11}}{\partial \Gamma_{2}}\right]}{E_{g}\left(\Gamma_{2}-\Gamma_{1}\right)^{2}} \\
\frac{\partial S_{g r}}{\partial \Gamma_{s}} & =\frac{\Gamma_{1} \Gamma_{2}\left(W_{r 2}-W_{r 1}\right) \frac{\partial S_{11}}{\partial \Gamma_{s}}}{E_{g}\left(\Gamma_{2}-\Gamma_{1}\right)} \\
\frac{\partial S_{g r}}{\partial W_{r 1}} & =\frac{\Gamma_{2}\left[1-\Gamma_{1} S_{11}+\Gamma_{1}\left(W_{r 2}-W_{r 1}\right) \frac{\partial S_{11}}{\partial W_{r 1}}\right]}{E_{g}\left(\Gamma_{2}-\Gamma_{1}\right)} \\
\frac{\partial S_{g r}}{\partial W_{r 2}} & =\frac{\Gamma_{2}\left[-1+\Gamma_{2} S_{11}+\Gamma_{2}\left(W_{r 2}-W_{r 1}\right) \frac{\partial S_{11}}{\partial W_{r 2}}\right]}{E_{g}\left(\Gamma_{2}-\Gamma_{1}\right)} \\
\frac{\partial S_{g r}}{\partial W_{r s}} & =\frac{\Gamma_{1} \Gamma_{2}\left(W_{r 2}-W_{r 1}\right) \frac{\partial S_{11}}{\partial W_{r s}}}{E_{g}\left(\Gamma_{2}-\Gamma_{1}\right)}
\end{aligned}
$$

The possible error in determining $S_{g r}$ parameter is of the form,

$$
\begin{aligned}
\left|\Delta S_{g r}\right|= & \left|\frac{\partial S_{g r}}{\partial \Gamma_{1}} \Delta \Gamma_{1}\right|+\left|\frac{\partial S_{g r}}{\partial \Gamma_{2}} \Delta \Gamma_{2}\right|+\left|\frac{\partial S_{g r}}{\partial \Gamma_{s}} \Delta \Gamma_{s}\right|+\left|\frac{\partial S_{g r}}{\partial W_{r 1}} \Delta W_{r 1}\right|+\left|\frac{\partial S_{g r}}{\partial W_{r 2}} \Delta W_{r 2}\right| \\
& +\left|\frac{\partial S_{g r}}{\partial W_{r s}} \Delta W_{r s}\right|
\end{aligned}
$$

From the $S_{g l} S_{1 r}$ expression, we get 
$\frac{\partial S_{g 1} S_{1 r}}{\partial \Gamma_{1}}=\frac{1}{\Gamma_{1}^{2}}\left(S_{g r}-\frac{W_{r 1}}{E_{g}}\right)+\left(S_{11}-\frac{1}{\Gamma_{1}}\right) \frac{\partial S_{g r}}{\partial \Gamma_{1}}+\left(S_{g r}-\frac{W_{r 1}}{E_{g}}\right) \frac{\partial S_{11}}{\partial \Gamma_{1}}$

$\frac{\partial S_{g 1} S_{1 r}}{\partial \Gamma_{2}}=\left(S_{11}-\frac{1}{\Gamma_{1}}\right) \frac{\partial S_{g r}}{\partial \Gamma_{2}}+\left(S_{g r}-\frac{W_{r 1}}{E_{g}}\right) \frac{\partial S_{11}}{\partial \Gamma_{2}}$

$\frac{\partial S_{g 1} S_{1 r}}{\partial \Gamma_{s}}=\left(S_{11}-\frac{1}{\Gamma_{1}}\right) \frac{\partial S_{g r}}{\partial \Gamma_{s}}+\left(S_{g r}-\frac{W_{r 1}}{E_{g}}\right) \frac{\partial S_{11}}{\partial \Gamma_{s}}$

$\frac{\partial S_{g 1} S_{1 r}}{\partial W_{r 1}}=\frac{1}{E_{g}}\left(\frac{1}{\Gamma_{1}}-S_{11}\right)+\left(S_{11}-\frac{1}{\Gamma_{1}}\right) \frac{\partial S_{g r}}{\partial W_{r 1}}+\left(S_{g r}-\frac{W_{r 1}}{E_{g}}\right) \frac{\partial S_{11}}{\partial W_{r 1}}$

$\frac{\partial S_{g 1} S_{1 r}}{\partial W_{r 2}}=\left(S_{11}-\frac{1}{\Gamma_{1}}\right) \frac{\partial S_{g r}}{\partial W_{r 2}}+\left(S_{g r}-\frac{W_{r 1}}{E_{g}}\right) \frac{\partial S_{11}}{\partial W_{r 2}}$

$\frac{\partial S_{g 1} S_{1 r}}{\partial W_{r s}}=\left(S_{11}-\frac{1}{\Gamma_{1}}\right) \frac{\partial S_{g r}}{\partial W_{r s}}+\left(S_{g r}-\frac{W_{r 1}}{E_{g}}\right) \frac{\partial S_{11}}{\partial W_{r s}}$

The possible error in determining the $S_{g 1} S_{t r}$ is

$$
\begin{aligned}
\left|\Delta S_{g 1} S_{1 r}\right|= & \left|\frac{\partial S_{g 1} S_{1 r}}{\partial \Gamma_{1}} \Delta \Gamma_{1}\right|+\left|\frac{\partial S_{g 1} S_{1 r}}{\partial \Gamma_{2}} \Delta \Gamma_{2}\right|+\left|\frac{\partial S_{g 1} S_{1 r}}{\partial \Gamma_{s}} \Delta \Gamma_{s}\right|+\left|\frac{\partial S_{g 1} S_{1 r}}{\partial W_{r 1}} \Delta W_{r 1}\right| \\
& +\left|\frac{\partial S_{g 1} S_{1 r}}{\partial W_{r 2}} \Delta W_{r 2}\right|+\left|\frac{\partial S_{g 1} S_{1 r}}{\partial W_{r s}} \Delta W_{r s}\right|
\end{aligned}
$$

Using HP sampling oscilloscope within the bandwidth, $\mathrm{S}_{11} \simeq 0, S_{g r} \simeq 1$, and $\mathrm{S}_{g 1} S_{t r} \simeq 1$. For the optimum calibration standard set, $\Gamma=-1,1$, and 0 . From equations (5.3.4) to (5.3.6), we have

$$
\begin{aligned}
& \left|\Delta S_{11}\right|=\left|-\frac{1}{2} \Delta \Gamma_{1}\right|+\left|-\frac{1}{2} \Delta \Gamma_{2}\right|+\left|\Delta \Gamma_{s}\right|+\left|\frac{1}{2} \Delta W_{r 1}\right|+\left|\frac{1}{2} \Delta W_{r 2}\right|+\left|-\Delta W_{r s}\right|, \\
& \left|\Delta S_{g r}\right|=\left|-\Delta \Gamma_{s}\right|+\left|\Delta W_{r s}\right|,
\end{aligned}
$$


and

$\left|\Delta S_{g 1} S_{1 r}\right|=\left|\frac{1}{2} \Delta \Gamma_{1}\right|+\left|-\frac{1}{2} \Delta \Gamma_{2}\right|+\left|-\frac{1}{2} \Delta W_{r 1}\right|+\left|\frac{1}{2} \Delta W_{r 2}\right|$

For a given TDNA system, the measurement error of the system, $\Delta W$, is given and will not change during the entire measurement procedure. The error for determining the the calibration standards, $\Delta \Gamma$, is in the order of $10^{-4}$ which can be neglected compared to the measurement error, $\Delta W$ in the order of $10^{-3}$. Substituting these into the above equations, we have

$\left|\Delta S_{11}\right|=2 \cdot|\Delta W|$

$\left|\Delta S_{g r}\right|=|\Delta W|$

and

$\left|\Delta S_{g 1} S_{1 r}\right|=|\Delta W|$

These equations set the error bounds for determining TDR model parameters.

\subsection{TDR DE-EMBEDDING ERRORS}

After the TDR model parameters have been attained, the model can be used to deembed the TDR measurement in order to improve the measurement accuracy. The basic equation for this de-embedding is of the form, 
$\Gamma_{d u t}=\frac{\frac{W_{d v t}}{E_{g}}-S_{g r}}{\left(\frac{W_{d u t}}{E_{g}}-S_{g r}\right) S_{11}+S_{g t} S_{1 r}}$

In order to analyze the de-embedding errors, the derivatives of equation (5.4.1) are incorporated to the model parameters and measurement data, respectively. From the $\Gamma_{d u t}$ expression, one can get,

$$
\begin{aligned}
& \frac{\partial \Gamma_{d u t}}{\partial W_{d u t}}=\frac{S_{g 1} S_{1 \mathrm{r}}}{\left[\left(\frac{W_{d u t}}{E_{g}}-S_{g r}\right) S_{11}+S_{g 1} S_{1 \mathrm{r}}\right]^{2}}, \\
& \frac{\partial \Gamma_{d u t}}{\partial S_{g r}}=\frac{-S_{g 1} S_{t \mathrm{r}}}{\left[\left(\frac{W_{d u t}}{E_{g}}-S_{g r}\right) S_{11}+S_{g 1} S_{1 r}\right]^{2}}, \\
& \frac{\partial \Gamma_{d u t}}{\partial S_{11}}=\frac{-\left(\frac{W_{d u t}}{E_{g}}-S_{g r}\right)^{2}}{\left[\left(\frac{W_{d u t}}{E_{g}}-S_{g r}\right) S_{11}+S_{g 1} S_{t r}\right]^{2}},
\end{aligned}
$$

and

$$
\frac{\partial \Gamma_{d u t}}{\partial\left(S_{g 1} S_{1 \tau}\right)}=\frac{-\left(\frac{W_{d u t}}{E_{g}}-S_{g r}\right)}{\left[\left(\frac{W_{d x t}}{E_{g}}-S_{g r}\right) S_{11}+S_{g 1} S_{1 r}\right]^{2}} .
$$

Then, the possible error in determining the $\Gamma_{d u t}$ is equal to,

$$
\left|\Delta \Gamma_{d u t}\right|=\left|\frac{\partial \Gamma_{d u t}}{\partial W_{d u t}} \Delta W_{d u t}\right|+\left|\frac{\partial \Gamma_{d u t}}{\partial S_{g r}} \Delta S_{g r}\right|+\left|\frac{\partial \Gamma_{d u t}}{\partial S_{11}} \Delta S_{11}\right|+\left|\frac{\partial \Gamma_{d u t}}{\partial\left(S_{g 1} S_{1 r}\right)} \Delta\left(S_{g 1} S_{1 r}\right)\right|
$$

Using the same conditions as in section (5.3), equations (5.3.7) to (5.3.9) can be used to calculate the de-embedding error. Then, equation (5.4.2) becomes $\left|\Delta \Gamma_{d x t}\right|=\left|\Delta W_{d u t}\right|+\left|-\Delta S_{g r}\right|+\left|-\left(1-W_{d v t}\right)^{2} \Delta S_{11}\right|+\left|\left(1-W_{d u t}\right) \Delta\left(S_{g 1} S_{1 r}\right)\right|$ 


$$
\begin{aligned}
= & |\Delta W|+|\Delta W|+2\left(1-W_{d v t}\right)^{2}|\Delta W| \\
& +\left(1-W_{d x t}\right)|\Delta W| \\
= & \left(5-5 W_{d x t}+2 W_{d v t}{ }^{2}\right)|\Delta W| .
\end{aligned}
$$

Since the minimum value of $W_{d x t}$ is zero, the maximum error for the de-embedding process is of the form,

$\left|\Delta \Gamma_{d u t}\right|=5|\Delta W|$.

This equation sets the error bound for determining the reflection coefficient of a device under test.

\subsection{TDT MODELING ERRORS}

For the obtained TDT model, the model parameters relations can be determined through a TDR and a TDT measurement connecting channel 1 and channel 2 directly of the scope. Denoting the TDR measurement as $\mathrm{W}_{r / 4}$, and TDT measurement as $\mathrm{W}_{t}$, then the model parameters can be calculated as follows:

$S_{22}=\frac{\frac{W_{r 4}}{E_{g}}-S_{g r}}{S_{g 1} S_{1 r}-S_{11}\left(\frac{W_{r 4}}{E_{g}}-S_{g r}\right)}$

and 
$S_{g t} S_{2 t}=\frac{W_{4 t}}{E_{g}}\left(1-S_{11} S_{22}\right)$

In order to analyze the TDT modeling errors, we need to analyze the sensitivities of the TDT model parameters to the TDR model parameters and the measurement results. First, we take the derivatives of the model parameters to the TDR model parameters and TDT measurement, respectively. From the $S_{22}$ expression, we get

$\frac{\partial S_{22}}{\partial W_{r 4}}=\frac{\frac{S_{g 1} S_{1 r}}{E_{g}}}{\left[S_{g 1} S_{1 r}-S_{11}\left(\frac{W_{r 4}}{E_{g}}-S_{g r}\right)\right]^{2}}$,

$\frac{\partial S_{22}}{\partial\left(S_{g 1} S_{1 r}\right)}=\frac{-\left(\frac{W_{r 4}}{E_{g}}-S_{g r}\right)}{\left[S_{g t} S_{1 \tau}-S_{11}\left(\frac{W_{r 4}}{E_{g}}-S_{g r}\right)\right]^{2}}$

$\frac{\partial S_{22}}{\partial S_{11}}=\frac{\left(\frac{W_{r 4}}{E_{g}}-S_{g r}\right)^{2}}{\left[S_{g 1} S_{1 r}-S_{11}\left(\frac{W_{r 4}}{E_{g}}-S_{g r}\right)\right]^{2}}$,

and

$\frac{\partial S_{22}}{\partial S_{g r}}=\frac{-S_{g 1} S_{1 r}}{\left[S_{g 1} S_{1 r}-S_{11}\left(\frac{W_{r 4}}{E_{g}}-S_{g r}\right)\right]^{2}}$.

The possible error in determining the $S_{22}$ parameter should be the same as the TDR de-embedding. According to equation (5.4.3), it is

$\left|\Delta S_{22}\right|=5|\Delta W|$ 
From the $S_{22}$ expression, equation (5.5.2), we get

$\frac{\partial\left(S_{g 1} S_{1 \mathrm{r}}\right)}{\partial W_{t 4}}=\frac{1}{E_{g}}\left(1-S_{11} S_{2 q}\right)$

$\frac{\partial\left(S_{g 1} S_{1 \mathrm{r}}\right)}{\partial S_{11}}=-\frac{W_{t 4}}{E_{g}} S_{22}$, and

$\frac{\partial\left(S_{g 1} S_{1 r}\right)}{\partial S_{22}}=-\frac{W_{t 4}}{E_{g}} S_{11}$

The possible error in determining $S_{g t} S_{2 t}$ is

$\left|\Delta\left(S_{g 1} S_{2 t}\right)\right|=\left|\frac{\partial\left(S_{g 1} S_{2 t}\right)}{\partial W_{t 4}} \Delta W_{t 4}\right|+\left|\frac{\partial\left(S_{g 1} S_{2 t}\right)}{\partial S_{11}} \Delta S_{11}\right|+\left|\frac{\partial\left(S_{g 1} S_{2 t}\right)}{\partial S_{22}} \Delta S_{22}\right|$.

Using HP sampling oscilloscope within the bandwidth, $\mathrm{S}_{22} \simeq 0$, and $S_{g 1} S_{2 t} \simeq 1$. Using the previous obtained data, we have

$\left|\Delta\left(S_{g 1} S_{2 t}\right)\right|=|\Delta W|$

These equations set the error bounds for determining TDT model parameters.

\subsection{TDT DE-EMBEDDING ERRORS}

For the TDT de-embedding, we need to perform two measurements. First, acquire the waveform denoted as $W_{t}$ by inserting the DUT in the channel 1 and channel 2. Then, reverse the DUT and acquire the other waveform $W_{t 2}$. The basic equations 
for them are as follows.

$$
\begin{gathered}
W_{t 1}=\frac{S_{21 d u t} S_{g 1} S_{t 2}}{1-S_{11} S_{11 d u t}-S_{22} S_{22 d u t}+S_{11} S_{11 d z t} S_{22} S_{22 d u t}-S_{21 d u t} S_{11} S_{22} S_{12 d u t}} \\
W_{t 2}=\frac{S_{12 d u t} S_{g 1} S_{t 2}}{1-S_{11} S_{11 d u t}-S_{22} S_{22 d u t}+S_{11} S_{11 d u t} S_{22} S_{22 d u t}-S_{21 d u t} S_{11} S_{22} S_{12 d u t}}
\end{gathered}
$$

Since both $S_{11}$ and $S_{22}$ are small, we neglect the $S_{11} S_{22}$ term, Then equations (5.6.1) and (5.6.2) become

$$
\begin{gathered}
W_{t 1}=\frac{S_{21 d u t} S_{g 1} S_{t 2}}{1-S_{11} S_{11 d u t}-S_{22} S_{22 d u t}} \\
W_{t 2}=\frac{S_{12 d u t} S_{g 1} S_{t 2}}{1-S_{11} S_{11 d u t}-S_{22} S_{22 d u t}}
\end{gathered}
$$

The TDT model can be used to de-embed the TDT measurement to improve the measurement accuracy. The basic equation for this de-embedding is

$$
\begin{aligned}
& S_{21 d u t}=\frac{W_{t 1}\left(1-S_{11} S_{11 d u t}-S_{22} S_{22 d u t}\right)}{S_{g 1} S_{t 2}} \\
& S_{12 d u t}=\frac{W_{t 2}\left(1-S_{11} S_{11 d u t}-S_{22} S_{22 d u t}\right)}{S_{g 1} S_{t 2}}
\end{aligned}
$$

In order to analyze the de-embedding errors, we take the derivatives of the equation (5.6.5) to the model parameters and measurement data, respectively. From the $S_{21 d u t}$ expression, we get

$$
\frac{\partial S_{21 d u t}}{\partial W_{t 1}}=\frac{1-S_{11} S_{11 d u t}-S_{22} S_{22 d u t}}{S_{g 1} S_{t 2}},
$$

Chapter 5. Error Analysis 
$\frac{\partial S_{21 d u t}}{\partial S_{11}}=\frac{-W_{t 1} S_{11 d u t}}{S_{g 1} S_{t 2}}$

$\frac{\partial S_{21 d u t}}{\partial S_{11 d x t}}=\frac{-W_{11} S_{11}}{S_{g 1} S_{t 2}}$

$\frac{\partial S_{21 d u t}}{\partial S_{22}}=\frac{-W_{t 1} S_{22 d u t}}{S_{g 1} S_{t 2}}$,

$\frac{\partial S_{21 d u t}}{\partial S_{22 d u t}}=\frac{-W_{t 1} S_{22}}{S_{g 1} S_{t 2}}$, and

$\frac{\partial S_{21 d u t}}{\partial\left(S_{g 1} S_{t 2}\right)}=\frac{-W_{t 1}\left(1-S_{11} S_{11 d u t}-S_{22} S_{22 d u t}\right)}{\left(S_{g 1} S_{t 2}\right)^{2}}$

Then, the possible error in determining the $S_{21 d u t}$ is

$$
\begin{aligned}
\left|\Delta S_{21 d \mathrm{u} t}\right|= & \left|\frac{\partial S_{21 d u t}}{\partial W_{t 1}} \Delta W_{t 1}\right|+\left|\frac{\partial S_{21 d u t}}{\partial S_{11}} \Delta S_{11}\right|+\left|\frac{\partial S_{21 d u t}}{\partial S_{11 d \mathrm{u} t}} \Delta S_{11 d \mathrm{u} t}\right|+\left|\frac{\partial S_{21 d u t}}{\partial S_{22}} \Delta S_{22}\right|+ \\
& +\left|\frac{\partial S_{21 d u t}}{\partial S_{22 d u t}} \Delta S_{22 d u t}\right|+\left|\frac{\partial S_{21 d v t}}{\partial\left(S_{g 1} S_{t 2}\right)} \Delta\left(S_{g 1} S_{t 2}\right)\right|
\end{aligned}
$$

Using the same assumptions as in previous sections (5.6.7) can be used to calculate the de-embedding error. Then, equation (5.6.7) becomes

$\left|\Delta S_{21 d v t}\right|=|\Delta W|+\left|-W_{t 1} \Delta W\right|$

Since the maximum absolute value of $W_{t 1}$ for passive devices is one, then the maximum error of $\Delta S_{21 d x t}$ is 
$\left|\Delta S_{21 \mathrm{dut}}\right|=2|\Delta W|$.

Examining equation (5.6.4), we can see that it is the same function as equation (5.6.3). Similarly, we can have

$\left|\Delta S_{t 2 d x t}\right|=2|\Delta W|$.

This set of equations sets the error bounds for determining the transmission coefficients of a device under test. 


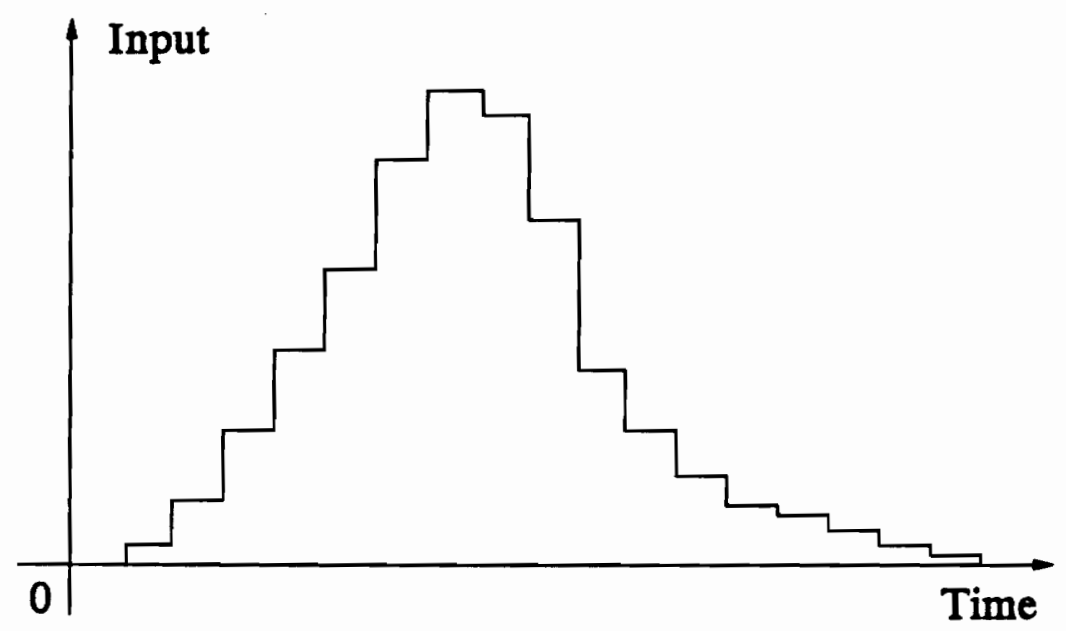

Figure 5.2.1 ADC input voltage. 


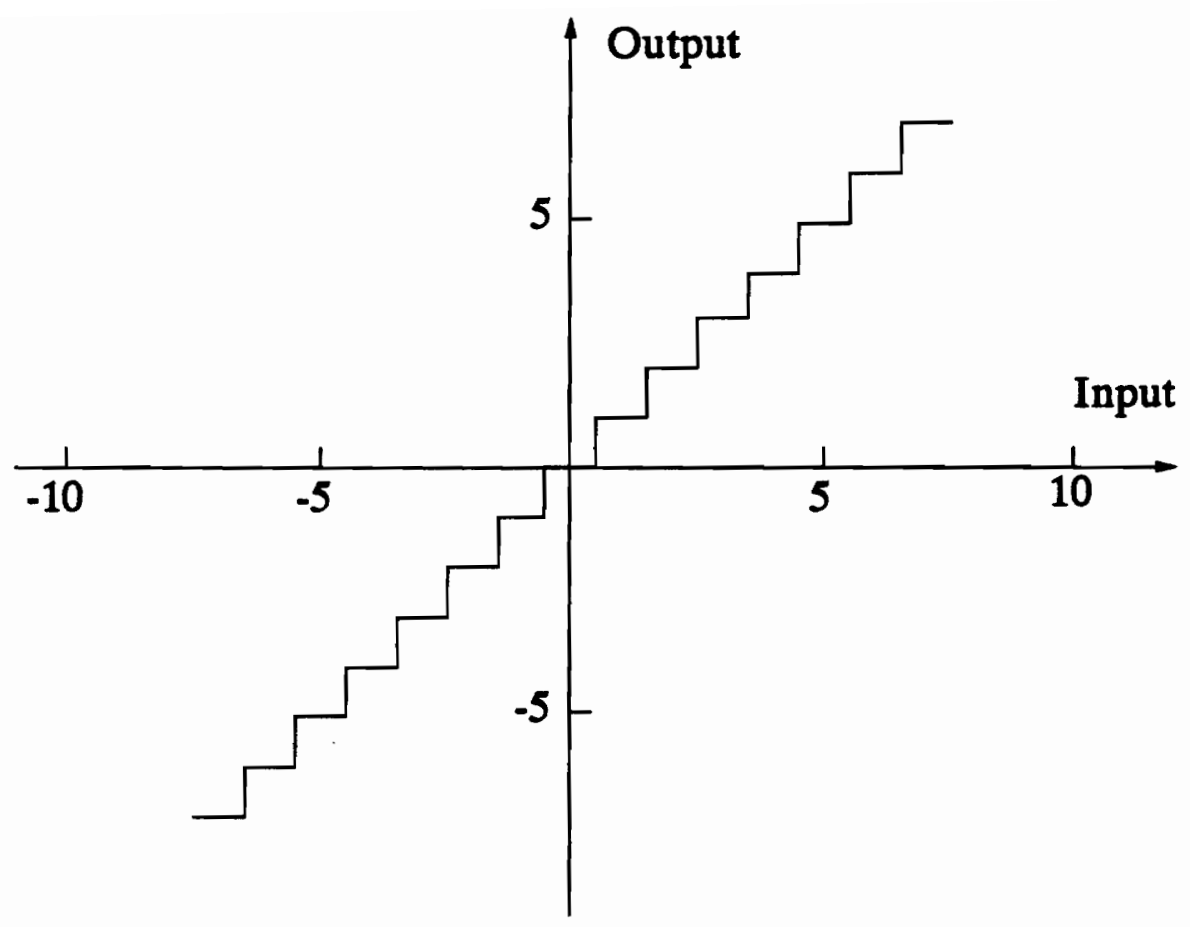

Figure 5.2.2 $\mathrm{ADC}$ transfer characteristics. 


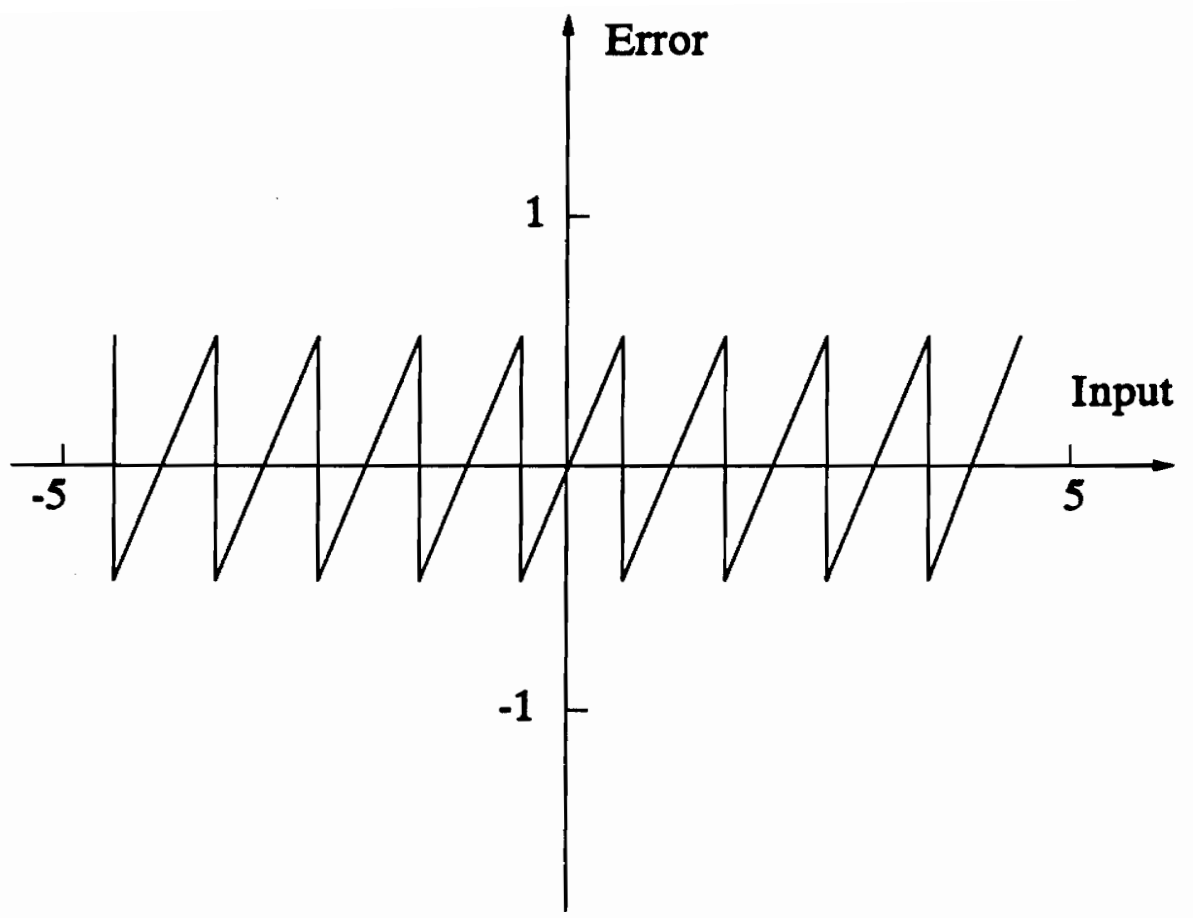

Figure 5.2.3 Quantization error versus input. 


\section{CHAPTER 6}

\section{EXPERIMENTAL RESULTS}

\subsection{INTRODUCTION}

In this chapter, system simulations are performed to verify the proposed calibration technique as well as evaluate potential error sources from practical measurement. In this regard, experimental procedures are described, and system modeling and deembedding are discussed [22], [23]. The obtained results are presented and compared to theoretically computed values to prove the validity of the calibration.

\subsection{SYSTEM SIMULATIONS}

Based on the system model developed, some simulations are performed to obtain general understanding about the model and model parameters.

\subsubsection{Software Program Verification}

Before the real calibration procedures are performed, the correctness of the 
calibration software programs should be checked. To achieve this, first, a step-like waveform is acquired from the oscilloscope. Then, the acquired waveform is used as an exciting pulse. From this exciting pulse, the ideal TDR and TDT responses for an ideal system to the short, open, load and through (direct connect) standards are simulated, as shown in Fig 6.2.1 for TDR responses and Fig. 6.2.2 for TDT response. After that, these simulated waveforms are fed into the modeling programs. The output of the modeling program is the model parameters. The obtained results for model parameters $S_{g r}, S_{g 1} S_{1 r}, S_{11}, S_{g t} S_{2 t}$ and $S_{22}$ are shown in Fig. 6.2.3 to 6.2.9. From the $S_{11}$ amplitude, see Fig. 6.2.6, the computation error is in the order of $10^{-7}$ which can be neglected comparing to the measurement error in the order of $10^{-3}$. Compare to the ideal system model parameters, the system modeling programs are conformed.

\subsubsection{Corrections for waveform time drift errors}

Due to the changing of environment conditions and component parameters, the time drifting among the acquired waveforms is inevitable in practical measurement systems. This phenomenon is simulated to check its effects on the system modeling. For doing so, the same set of simulated response waveforms is used except moving the open circuit response waveform one point backward and the short circuit response waveform one point forward. Figures 6.2.10 to 6.2.13 show the obtained results. These results reveal that the modeling process is very sensitive to this time drifting. In fact, shifting waveform one point corresponds to only $1.2 \mathrm{ps}$ time shifting in this simulation. From Fig. 6.2.11 and Fig. 6.2.13, however, significant errors are existed even under $30 \mathrm{GHz}$. So, it is important to 
align the all acquired waveforms. A program is developed to achieve the alignment between waveforms. The alignment is achieved from aligning the first transitions which are the same for all TDR waveforms. The alignment can reach 0.01 point using the mean square root value as the criterion. Figures 6.2 .14 and 6.2.15 show the results after adjusting the time shifting from one point to 0.01 point. Within the bandwidth of interest, the errors caused by this 0.01 point waveform shifting are negligible. For the TDT configuration, the waveform drifting is not so critical because this shifting only causes phase delay.

\subsection{EXPERIMENTAL CONSIDERATIONS}

In order to obtain good measurement results, measurements should be carefully arranged. For a time domain measurement system, a couple of contradictory factors should be considered. First the averaging number $N$, as seen in chapter 4 , can reduce the system noise to $1 / \sqrt{N}$. To reduce random noise, $N$ should be large. On the other hand, increasing $N$ implies longer time period needed to acquire a waveform. The longer time a measurement takes, the larger the waveform drifting would be. This waveform drifting will distort the waveform under test and degrade the measurement accuracy. A compromise must be made to optimize both signal noise level and waveform distortion. Second is the time window and time resolution. An acquired time domain waveform should include all the multiple reflections which contain useful information. This interprets a large time window to include the entire waveform. In contrast, describing detailed waveform structures needs small sampling time spacing or fine time resolution. However, the 
total sampling points in a time window is limited. The compromise has to be made between the time window and time resolution. The zero padding method discussed in Section 4.5 is used to enhance the frequency domain resolution. In all of the measurements, acquired time domain waveforms consist of 1000 point data. Then, the zero padding method is used to expand the measurement data to 4096 points. This process approximately quadruples the frequency domain resolution.

As mentioned before, environment conditions play an important role in obtaining good measurement results. Temperature is especially critical to measurement. Stabilizing the temperature and warming up the equipment are an important step. A set of optimum operating conditions is recommended for the HP sampling oscilloscopes [24]. $10 \mathrm{KHz}$ pulse repetition rate and 64 time averaging are found best for our system.

\subsection{MODELING TDNA SYSTEM}

Modeling involves both measuring the response waveforms from the calibration standards and evaluating the model parameters. The two procedures are as follows.

\subsubsection{Acquisition of the response waveforms}

First, the calibration standards (the offset short, offset open and matching load) are connected to the TDR terminal and TDR response waveforms to these standards 
are acquired, respectively. The obtained time domain waveforms are shown in Fig. 6.4.1 to Fig. 6.4.3. Then a flexible coaxial cable with male connectors is connected to the channel 2 terminal. This cable provides both extension and changing the connector sex. The cable should be always connected to channel 2 during the whole calibration and measurement procedure. Finally, connect the other end of the cable to TDR channel and measure the reflection from channel 1 and the transmission from channel 2. The obtained waveforms are shown in Fig. 6.4.4 and Fig. 6.4.5.

\subsubsection{Evaluation of model parameters}

After acquiring the response waveforms, the systematic errors like the scale errors and nonlinearities in the time and signal channels, need to be corrected. In our system, the nonlinearities are checked using DC voltage for the signal channel and linear rising ramp function for the time channel. Linear rising ramp is approximated by the crossing zero portion of a $20 \mathrm{MHz}$ sinusoidal signal from an HP 3325A synthesizer/function generator. Within the measurement range, both the nonlinearities are in the order of $0.1 \%$ and can be neglected in our application. Then the waveform data are transformed into frequency domain using Fourier transform. When transforming the step-like waveform using Fourier transform, some special algorithms are recommended [25]. Finally, the model parameters are evaluated using the equations (2.5.12) to (2.5.16). 


\subsection{DE-EMBEDDING}

Using the obtained system model, the measurement accuracy can be improved through the de-embedding. Before the practical de-embedding, the same short circuit waveform for the system calibration is used again to verify the correctness of the de-embedding program. Both acquired and de-embedded time domain waveforms are shown in Fig. 6.5.1. In this figure, the transient time of acquired waveform is about $50 \mathrm{ps}$. The observed transient time of the de-embedded short circuit response is $1.2 \mathrm{ps}$ which corresponds to the minimum acquisition time interval. The oscillations around the transition region is due to the Gibbs phenomenon. This result is no surprise since the measured short was one of the standards used in the calibration (the short was assumed ideal, $\Gamma=-1$ ). Ideally, a zero transition duration is expected to result from this computation. Due to digital and numerical limitations, the $1.2 \mathrm{ps}$ transition duration and the Gibbs oscillations are observed.

A $30 \Omega$ coaxial air line is used to show the TDR de-embedding. The reflection waveform from the $30 \Omega$ line is acquired, as shown in Fig. 6.5.2. Using the deembedding procedures mentioned above, the obtained responses are shown in Fig. 6.5.3 for amplitude and Fig. 6.5.4 for phase. Corresponding time domain response is illustrated in Fig. 6.5.5. An optimum filter [22] is applied to obtain smooth time domain step response. The variable of the filter $\gamma$ was choosing as 0.001 . The transition duration is less than $12 p s$ and the frequency of ringing in the waveform is about $40 \mathrm{GHz}$. The ringing before the transition is caused by the noncausal deconvolution filter and the noncausal model for the open circuit standard while 
high order modes is another possible reason for the ringing in the entire waveform.

A $10 d B$ coaxial attenuator is used to show the TDT de-embedding. The transmission waveform from the attenuator is acquired by channel 2, shown in Fig. 6.5.6. $S_{11 d u t}$ and $S_{22 d v t}$ of the attenuator were measured using TDR configuration. The de-embed response was evaluated using equations (5.6.5) and (5.6.6). Corresponding frequency and time domain results $(\gamma=0.001)$ are shown in Fig. 6.5.7 to 6.5.8. For the time domain step response, the transition duration is less than $15 \mathrm{ps}$. The preshoot before the major transition shows a noncausal behavior which is due to the signal processing. The preshoot and ringing in the waveform are caused by the noncausal deconvolution filter. The noncausal model offered by the manufacturer for the open circuit standard is another reason.

\subsection{VERIFICATION}

In order to verify the de-embedding results, measurements on the $30.56 \Omega$ coaxial air line were conducted using the calibrated TDR system. The line parameters were also calculated using the line geometry and material properties. The $30 \Omega$ coaxial line and $10 d B$ attenuator are also measured using frequency domain network analyzer, HP-8510B. Figure 6.6.1 shows the comparison among the three sets of results for the $30 \Omega$ coaxial line. Figures 6.5.7 shows the comparison between the time domain and the frequency domain results for the coaxial attenuator. The figures demonstrate an excellent agreement among the all data sets within $0.5 \mathrm{~dB}$ variations up to $20 \mathrm{GHz}$ bandwidth. 
To demonstrate the performance enhancement of the calibration technique, the three sets of results corresponding to measurements using "no calibration", "short circuit calibration" and "our TDNA calibration" are shown in Fig. 6.6.2. The "no calibration" result was obtained by numerically performing a differentiation and FFT on the measured response of the $30 \Omega$ line. The "short circuit calibration" starts by acquiring two response waveforms: the response waveform from the short circuit standard and the response waveform from the $30 \Omega$ line. Then, the ratio of the $30 \Omega$ line response to the short circuit response in frequency domain is taken as the de-embedded result for the $30 \Omega$ line. In all these measurements, the HP 54120 's normalization was not used. The figure illustrates that the $3 d B$ system bandwidth is about $8 \mathrm{GHz}$ without calibration. Using the simple short circuit calibration, the effective bandwidth for the results with adequate accuracy is about $10 \mathrm{GHz}$. The TDNA calibration technique increases the measurement bandwidth more than 2.5 times comparing to the result of no calibration and greatly enhances the measurement accuracy comparing to the simple short circuit calibration.

From the error analysis performed in Chapter 5, preliminary error bounds can be obtained for the measurement results. In order to find the error bounds, the system measurement error $\Delta W$ needs to be found first according to the equation (5.4.3). For the HP 54120T oscilloscope, there are two main error sources. One is the measurement accuracy which is $0.4 \%$ specified by the manufacturer. The other is noise. Figure 6.6.3 shows the normalized signal spectrum (upper trace) of the system and background noise spectrum (lower trace). The measurement error caused by noise is the ratio of these two spectra. As frequency increases, the signal spectrum amplitude decreases while the noise spectrum amplitude even increases. 
This makes the error be the dominant effects at high frequencies. Combining these two errors as $\Delta W$, we plot the error bounds for the TDR measurement as shown in Fig. 6.6.4. The error bounds are calculated using equation (5.4.3) and the curve fitting is used to obtain smooth error bound curves in Fig. 6.6.4. This figure further illustrates that the measurement accuracy is greatly limited by the $S / N$. Especially at high frequencies, the dominant error source is noise.

\subsection{SECONDARY CALIBRATION STANDARDS}

A set of secondary calibration standards can be established by using this calibrated TDNA. Using the measured data by the calibrated TDNA as the responses of the secondary standards can greatly reduce the work of modeling the standards as a cost of sacrificing the accuracy. It is acceptable for most of the industrial testing.

The secondary calibration standards can also be characterized using frequency domain VNA. First, a VNA is used to measure the standards. Then, a rational function approximation program [26] is used to obtain the rational function expressions for these standards. Inside the VNA bandwidth, the characteristics is well defined by the VNA measurements. Outside the bandwidth, the rational function is used to extend the frequency range. The important fact for using the rational function is that it always gives a causal model. The causality is critical to time domain responses. 


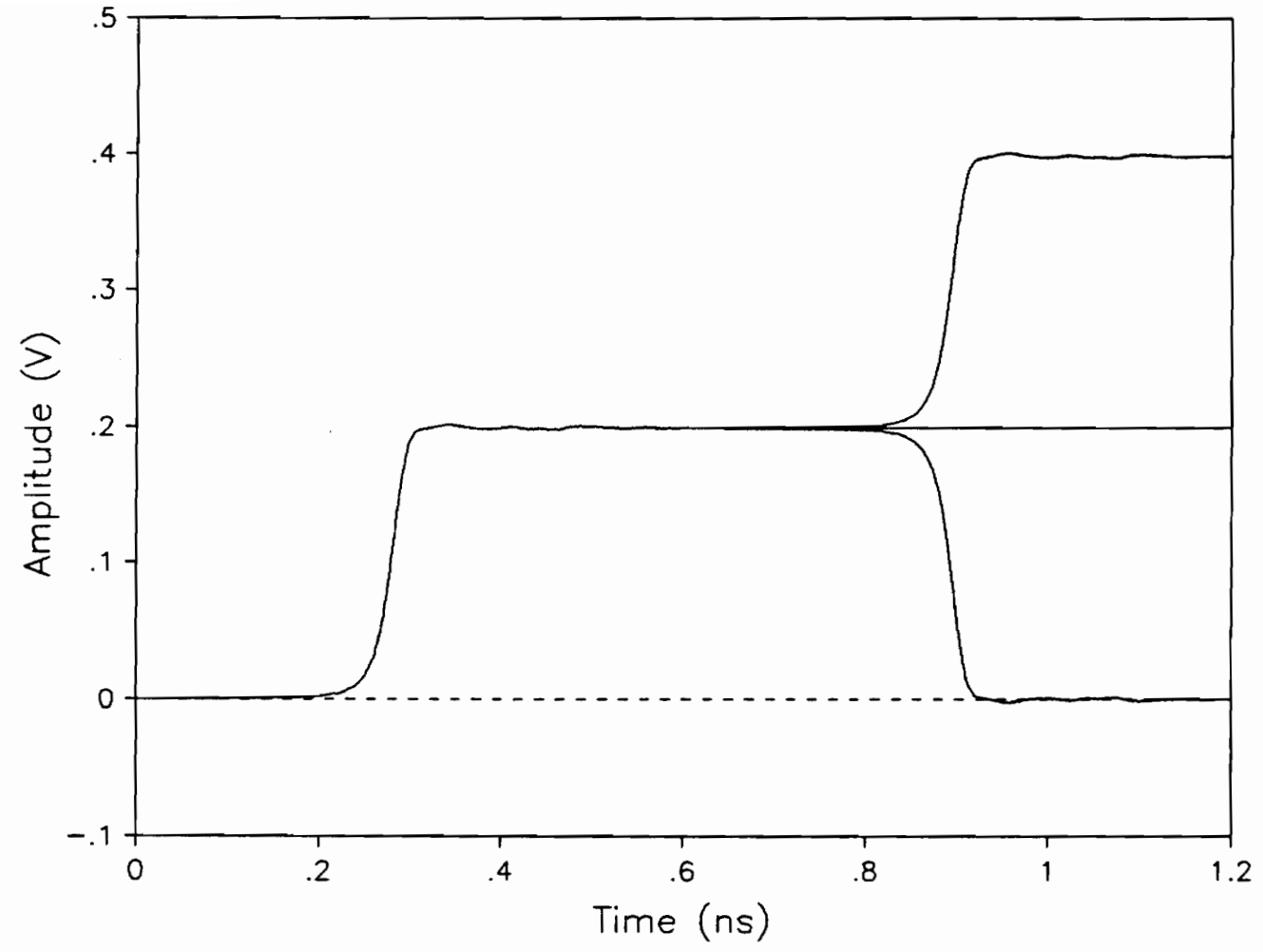

Figure 6.2.1 Simulated TDR waveforms. 


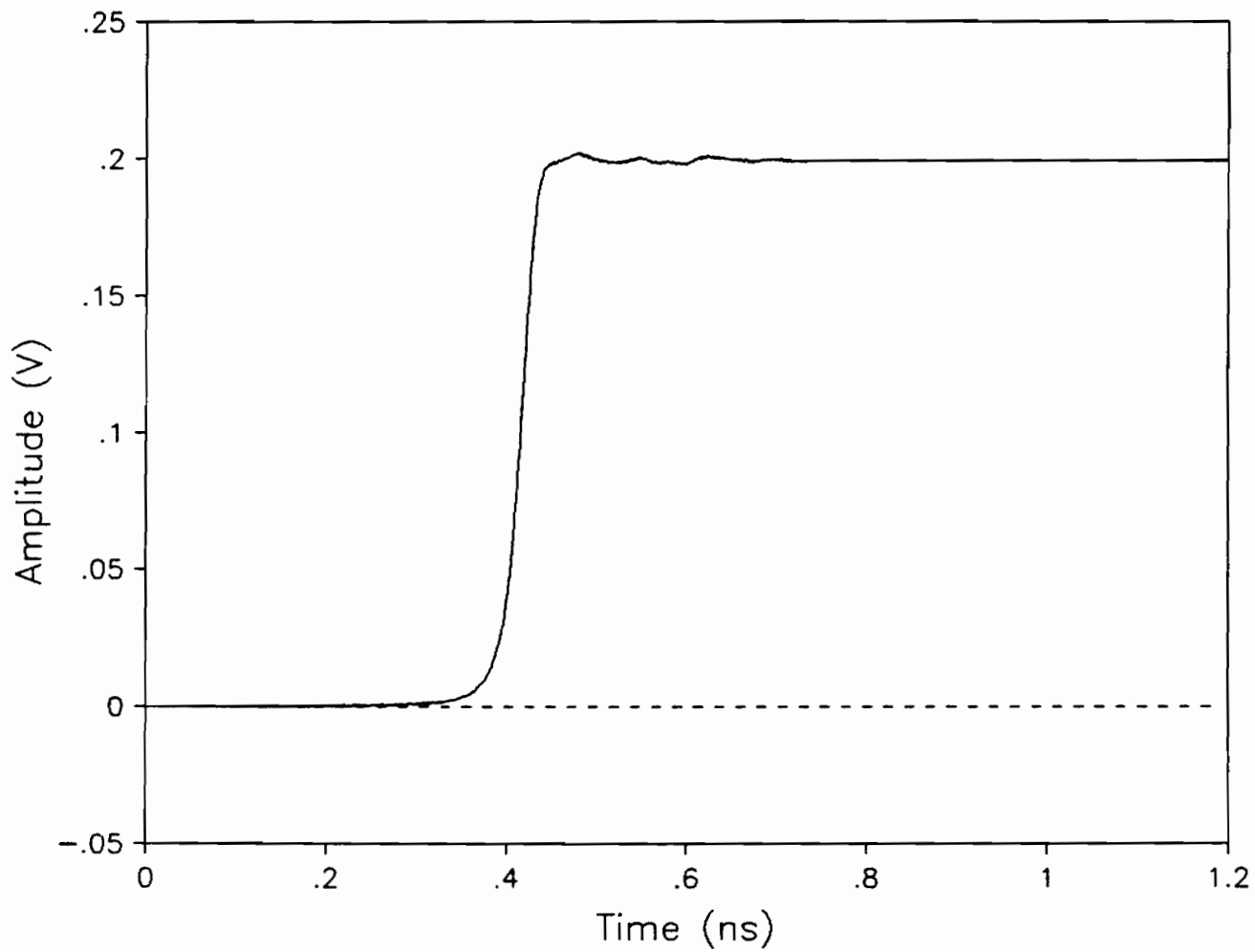

Figure 6.2.2 Simulated TDT waveform. 


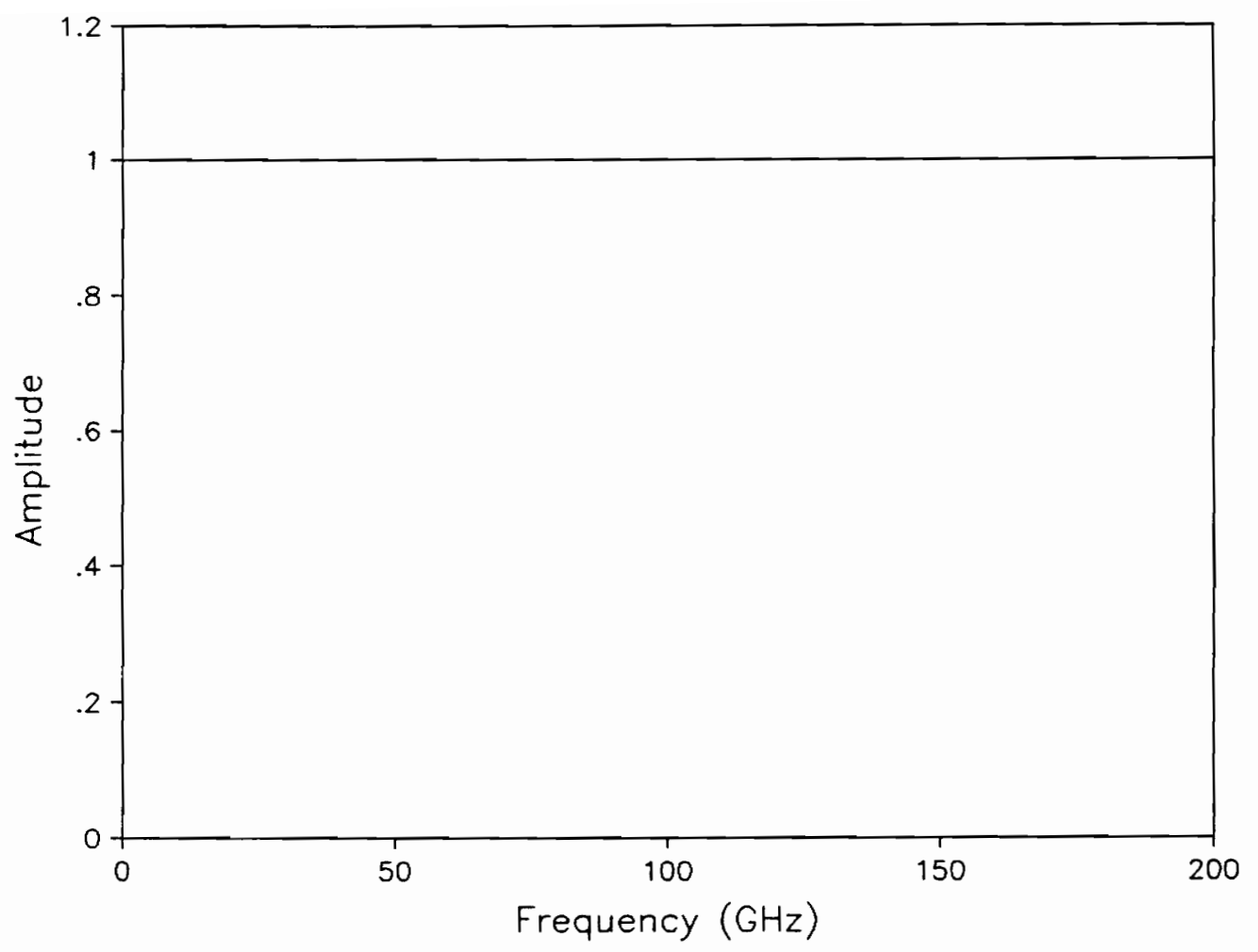

Figure 6.2.3 Results of the verification simulation:

$S_{g r}$ amplitude versus frequency. 


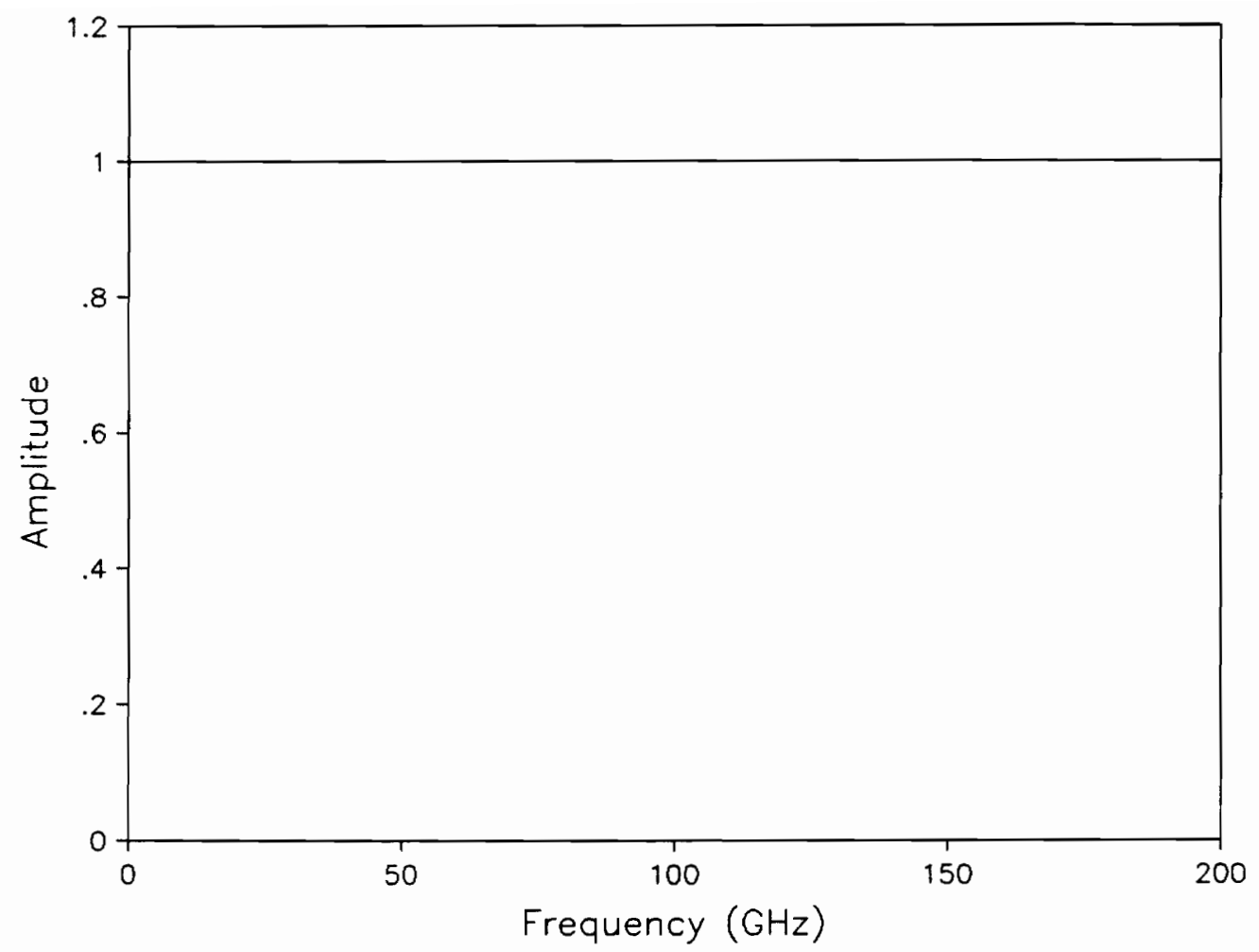

Figure 6.2.4 Results of the verification simulation:

$S_{g 1} S_{1 r}$ amplitude versus frequency. 


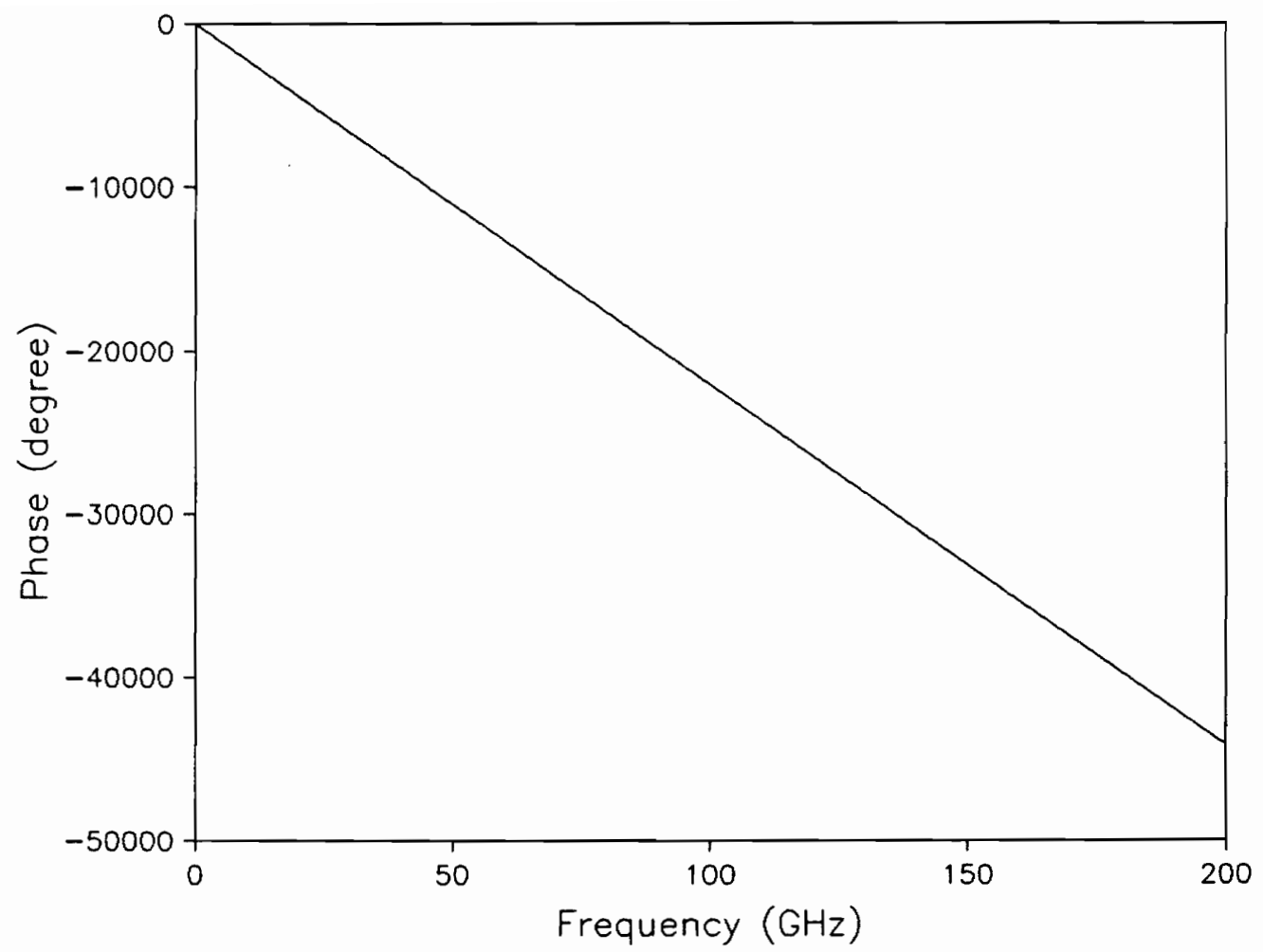

Figure 6.2.5 Results of the verification simulation:

$S_{g 1} S_{t r}$ phase versus frequency. 


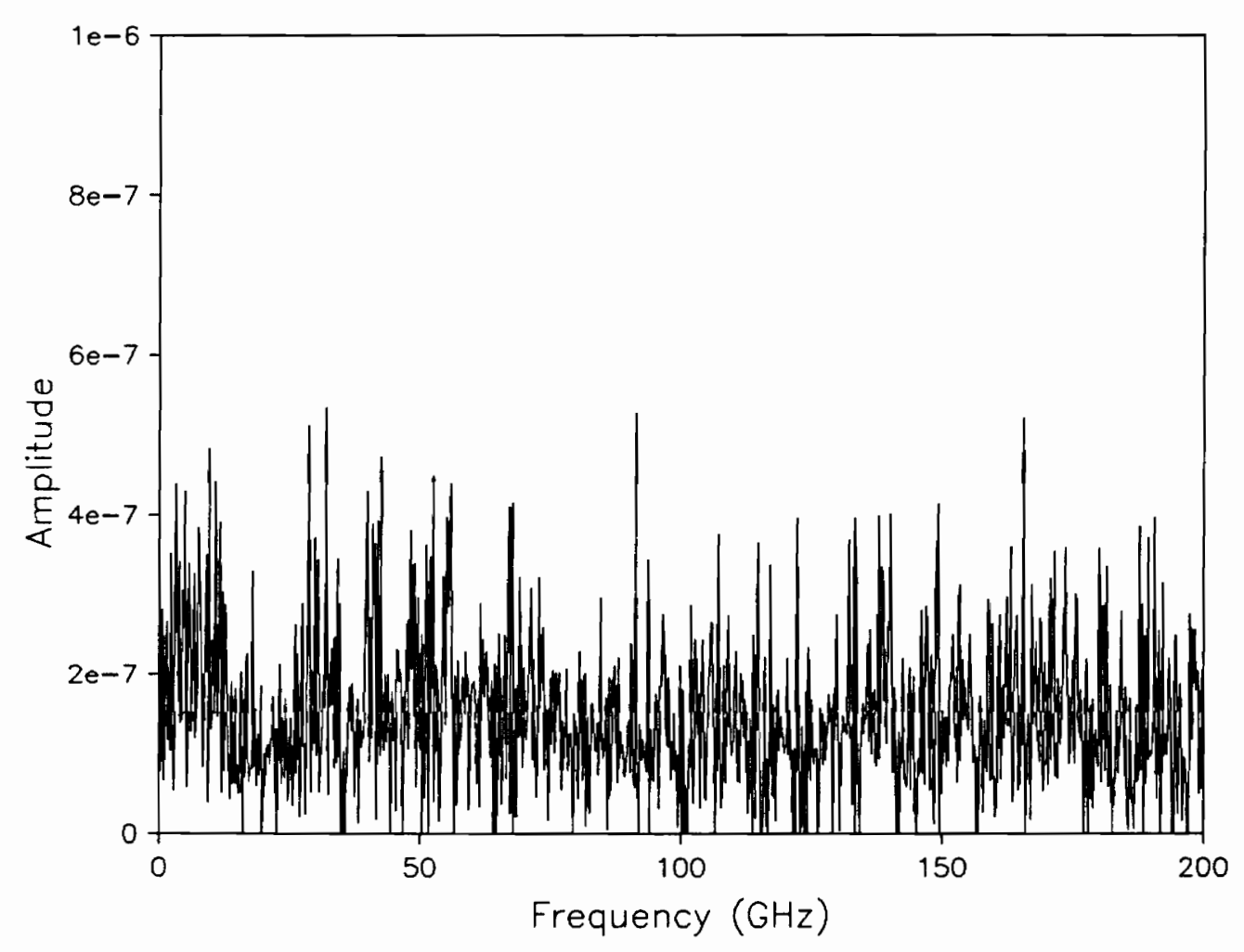

Figure 6.2.6 Results of the verification simulation:

$S_{11}$ amplitude versus frequency. 


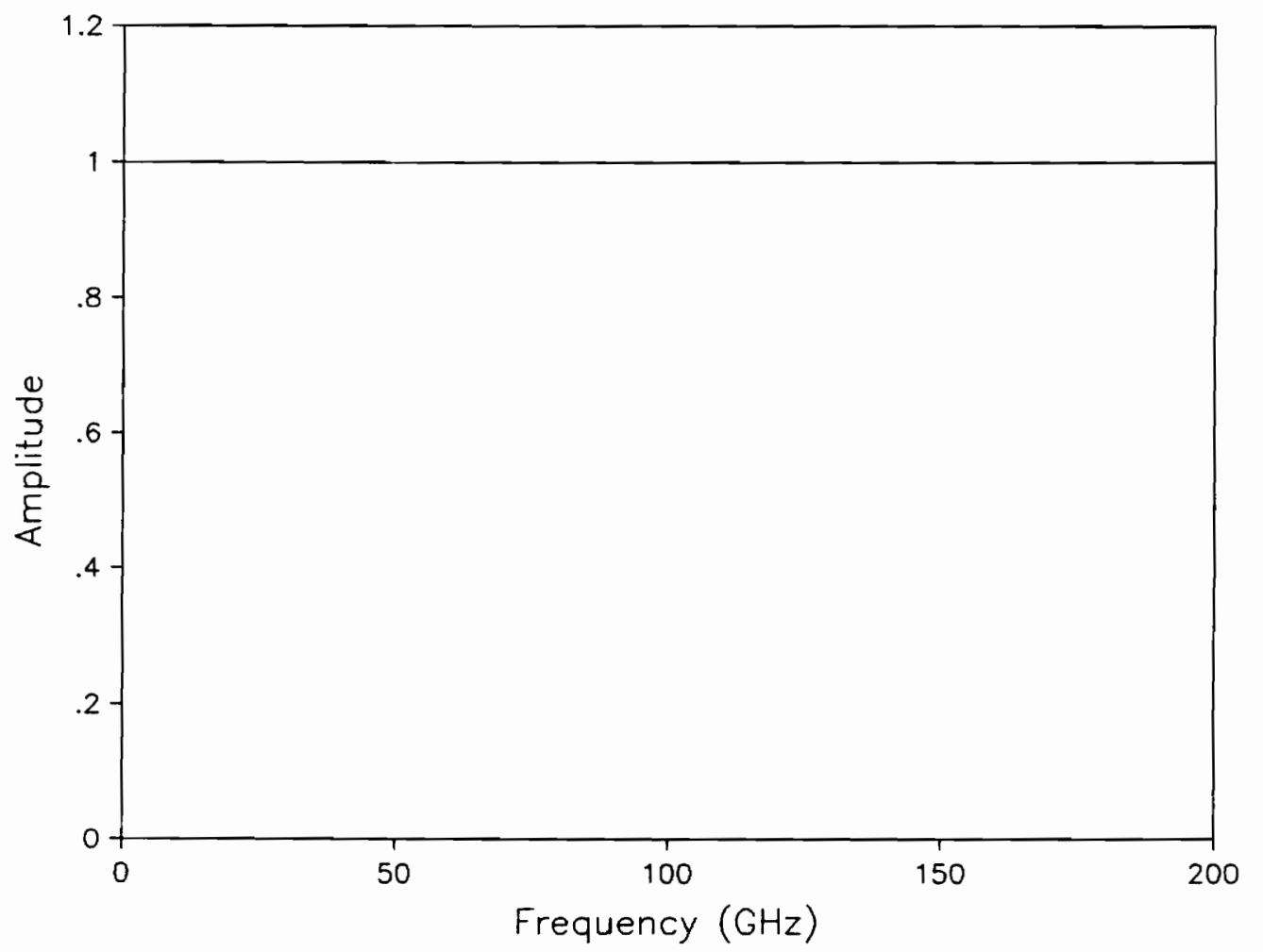

Figure 6.2.7 Results of the verification simulation:

$S_{g 1} S_{2 t}$ amplitude versus frequency. 


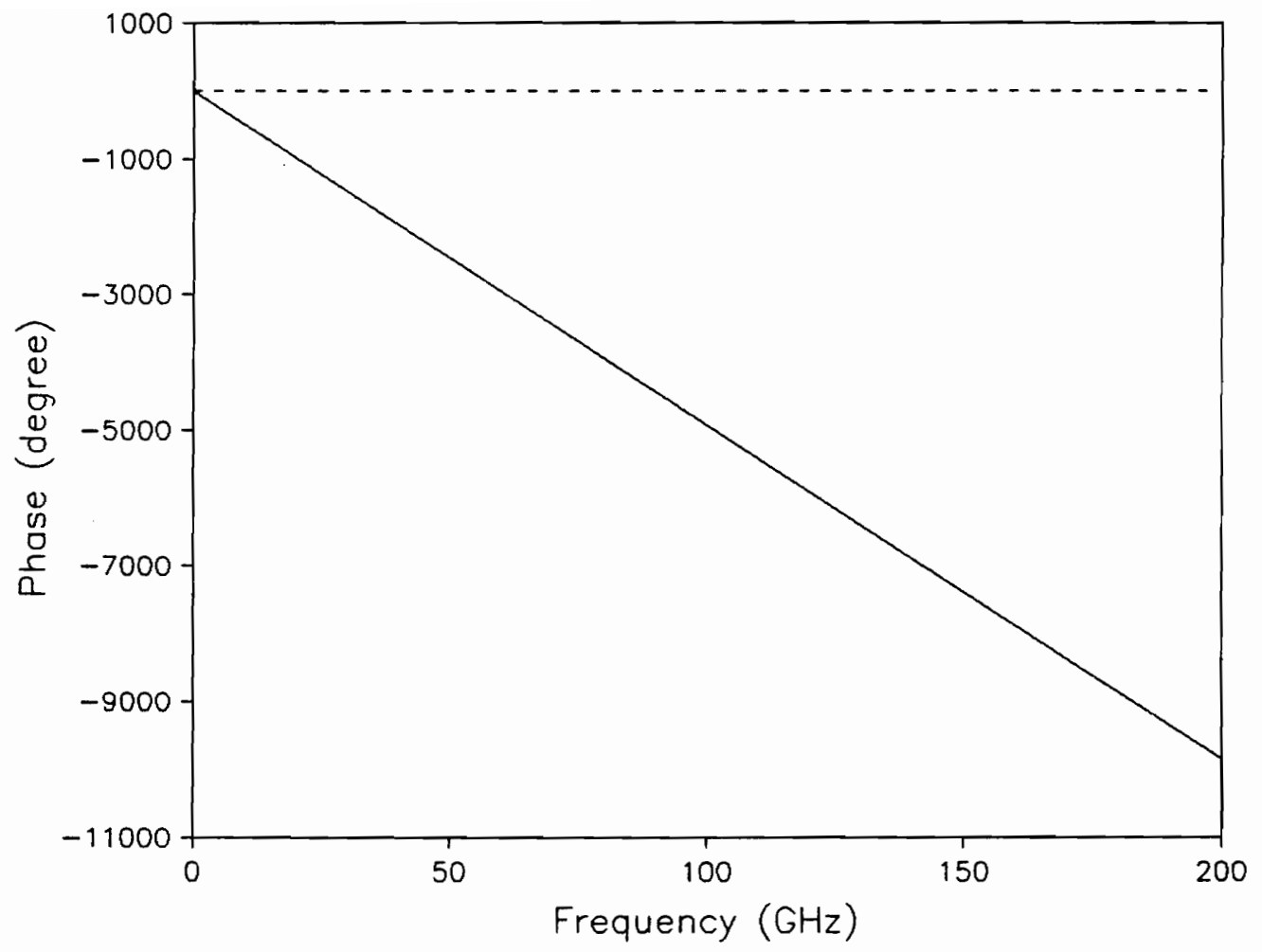

Figure 6.2.8 Results of the verification simulation: $S_{g 1} S_{2 t}$ phase versus frequency. 


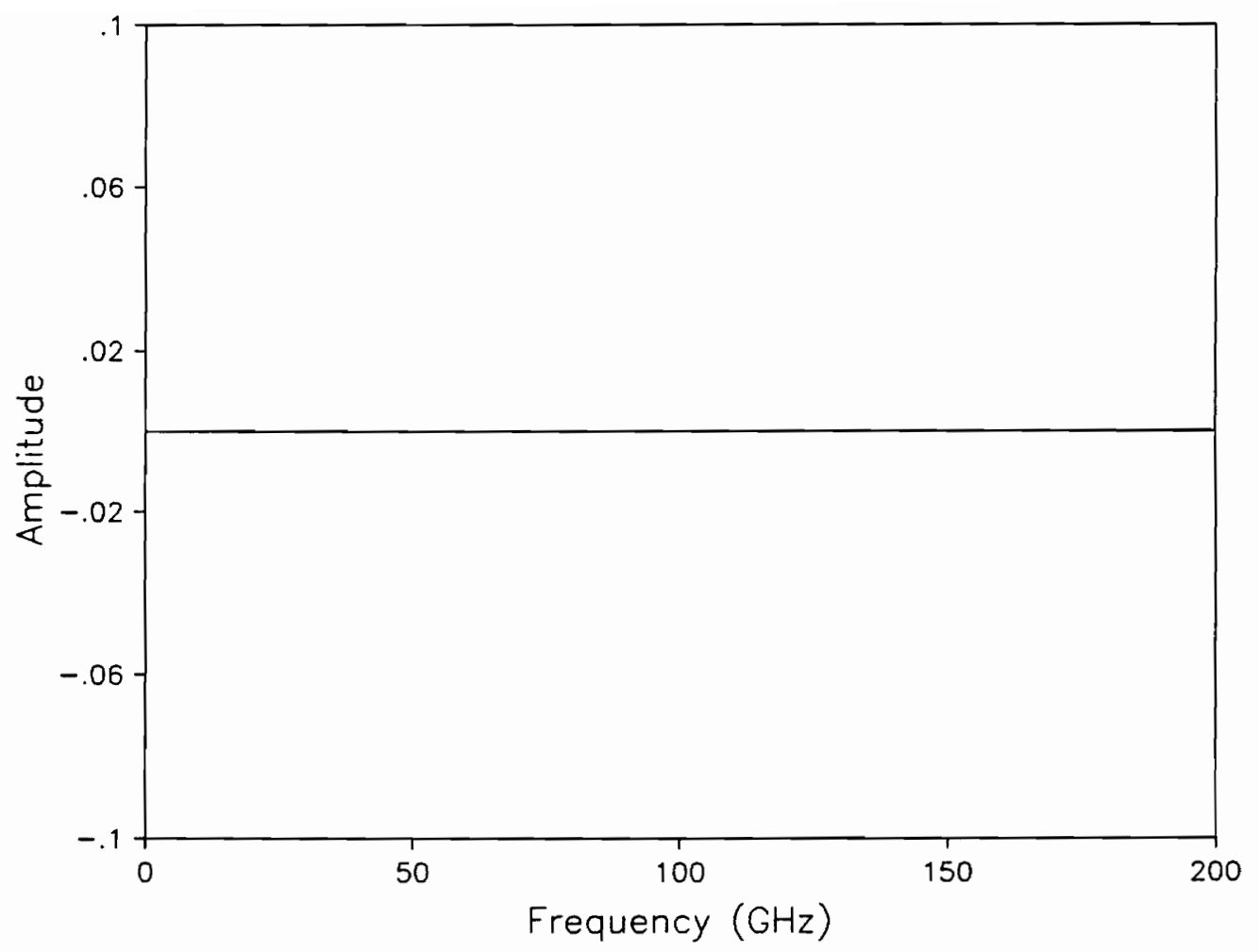

Figure 6.2.9 Results of the verification simulation:

$S_{22}$ amplitude versus frequency. 


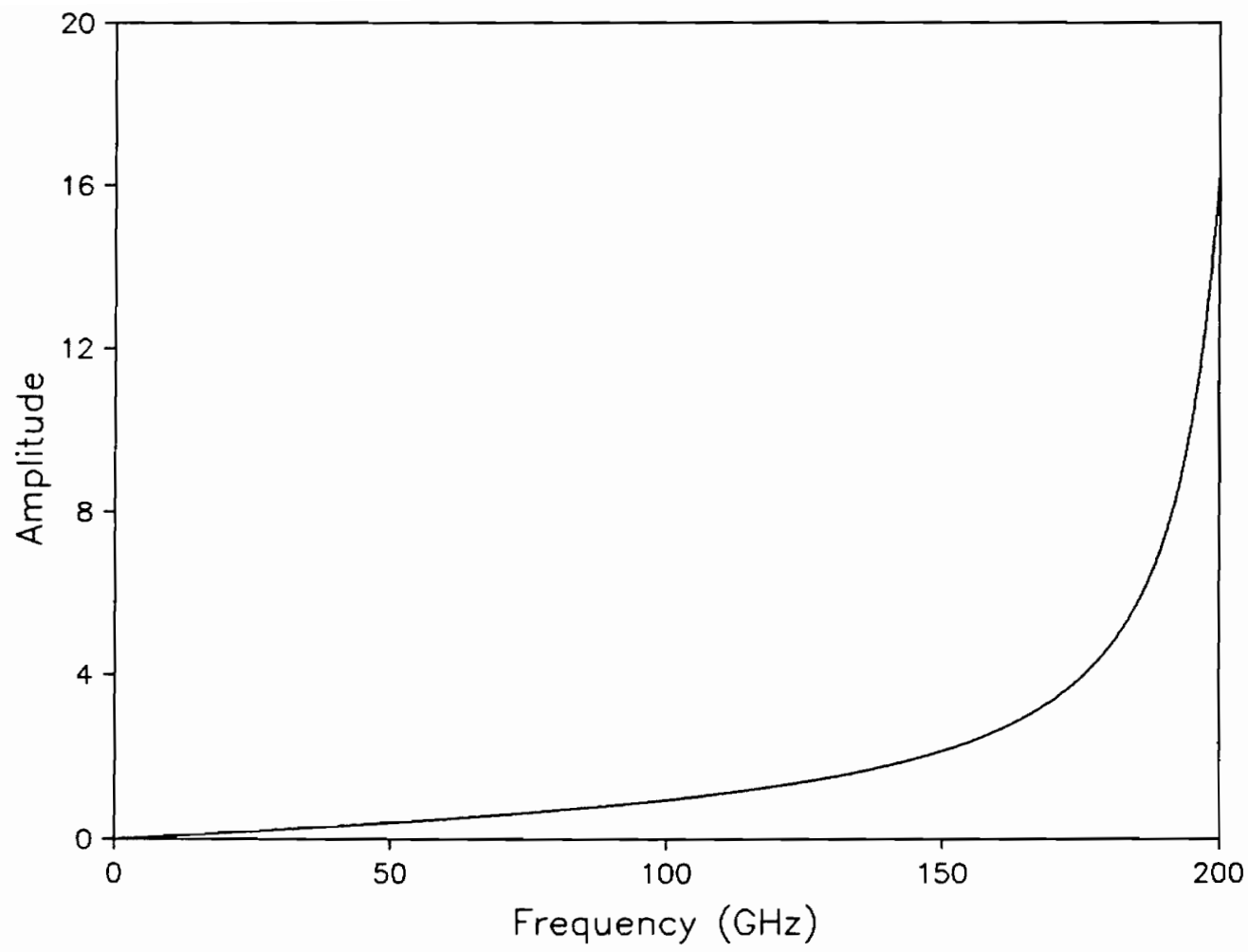

Figure 6.2.10 Results of the verification simulation: $S_{11}$ amplitude versus frequency (shifting the open circuit TDR waveform 1 point forward and the short circuit TDR waveform 1 point backward). 


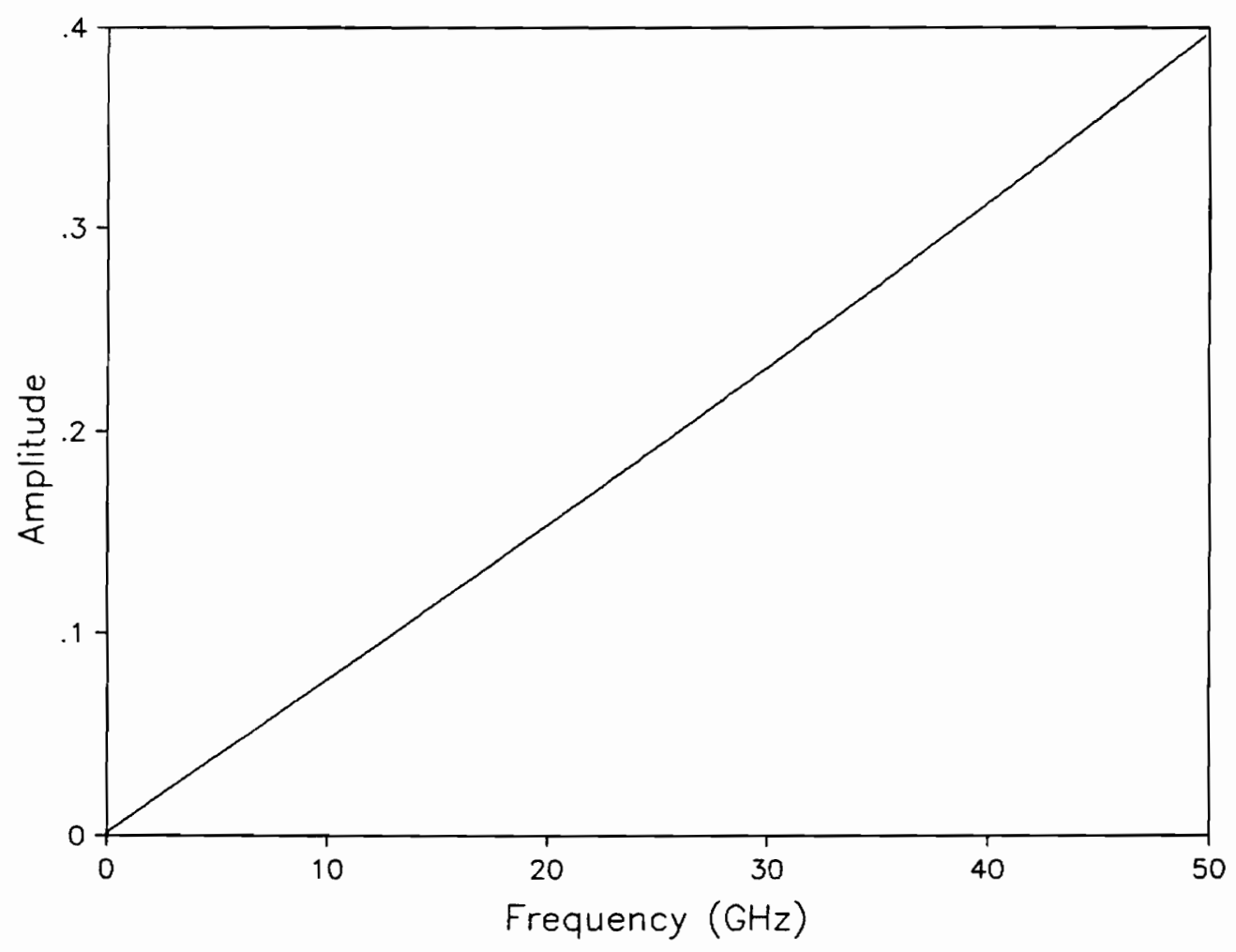

Figure 6.2.11 Results of the verification simulation: $S_{11}$ amplitude versus frequency (same as Fig. 6.2.10 except the scale of the frequency axis). 


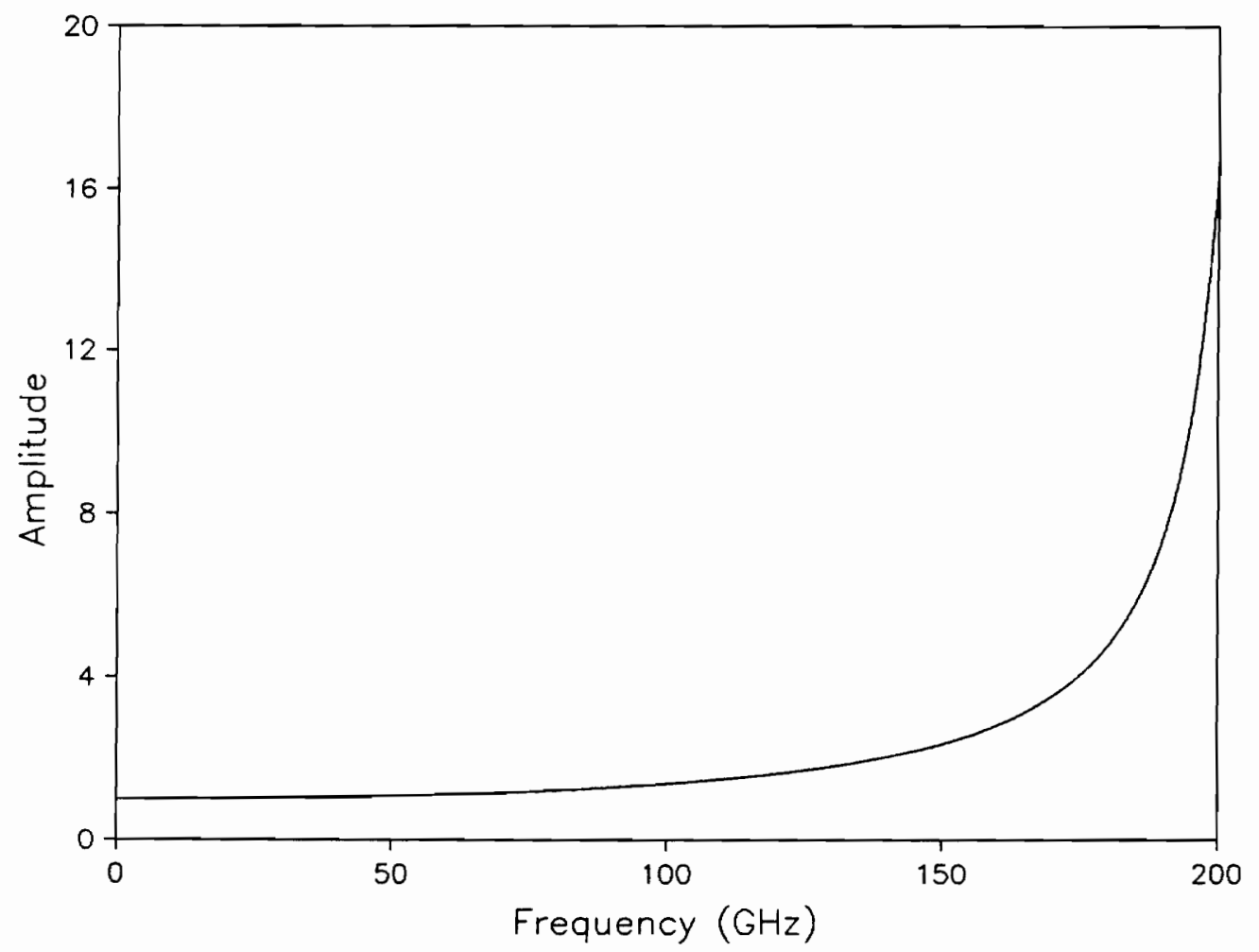

Figure 6.2.12 Results of the verification simulation: $S_{g 1} S_{1 r}$ amplitude versus frequency (shifting the open circuit TDR waveform 1 point forward and the short circuit TDR waveform 1 point backward). 


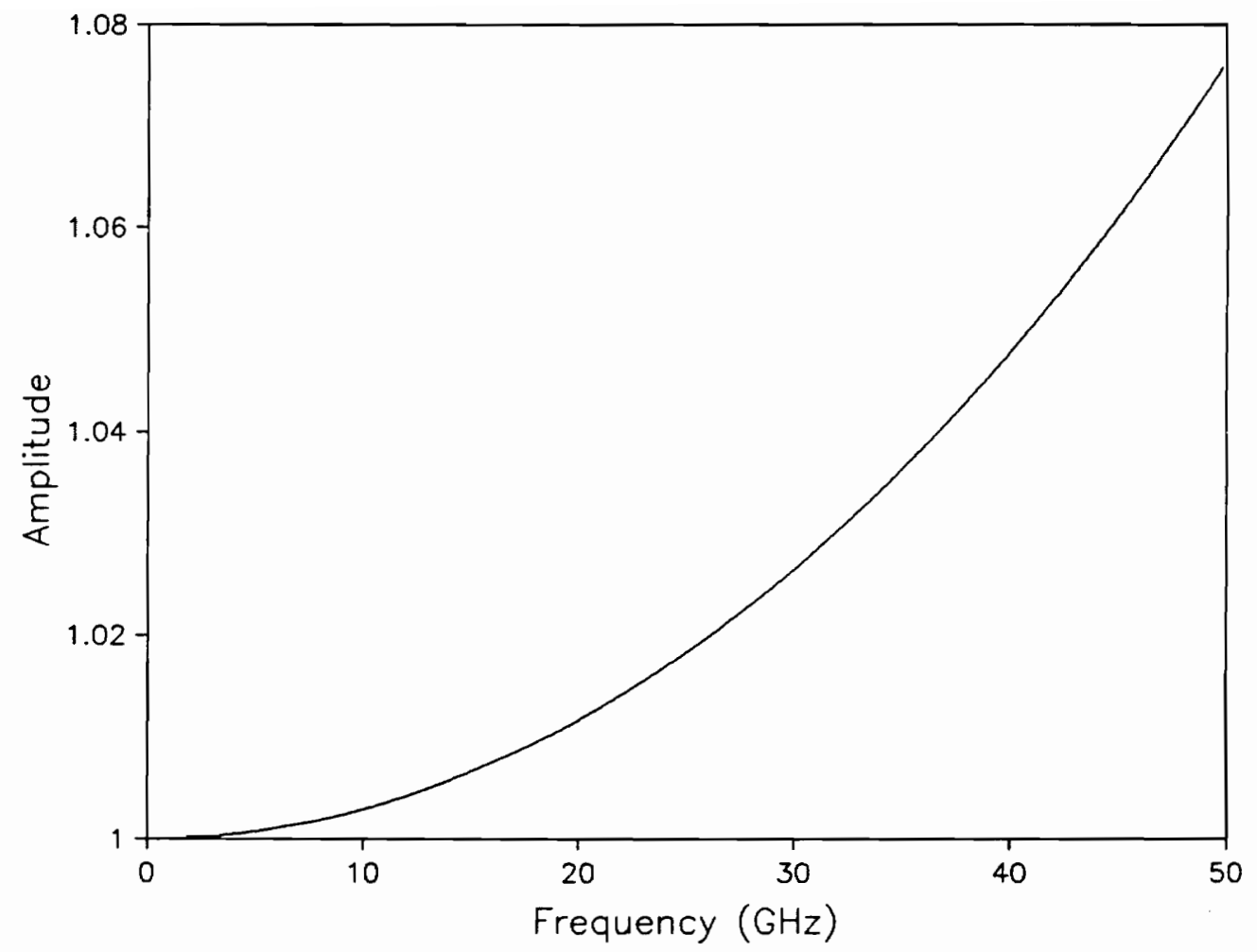

Figure 6.2.13 Results of the verification simulation: $S_{g 1} S_{1 r}$ amplitude versus frequency (same as Fig. 6.2.12 except the scale of the frequency axis). 


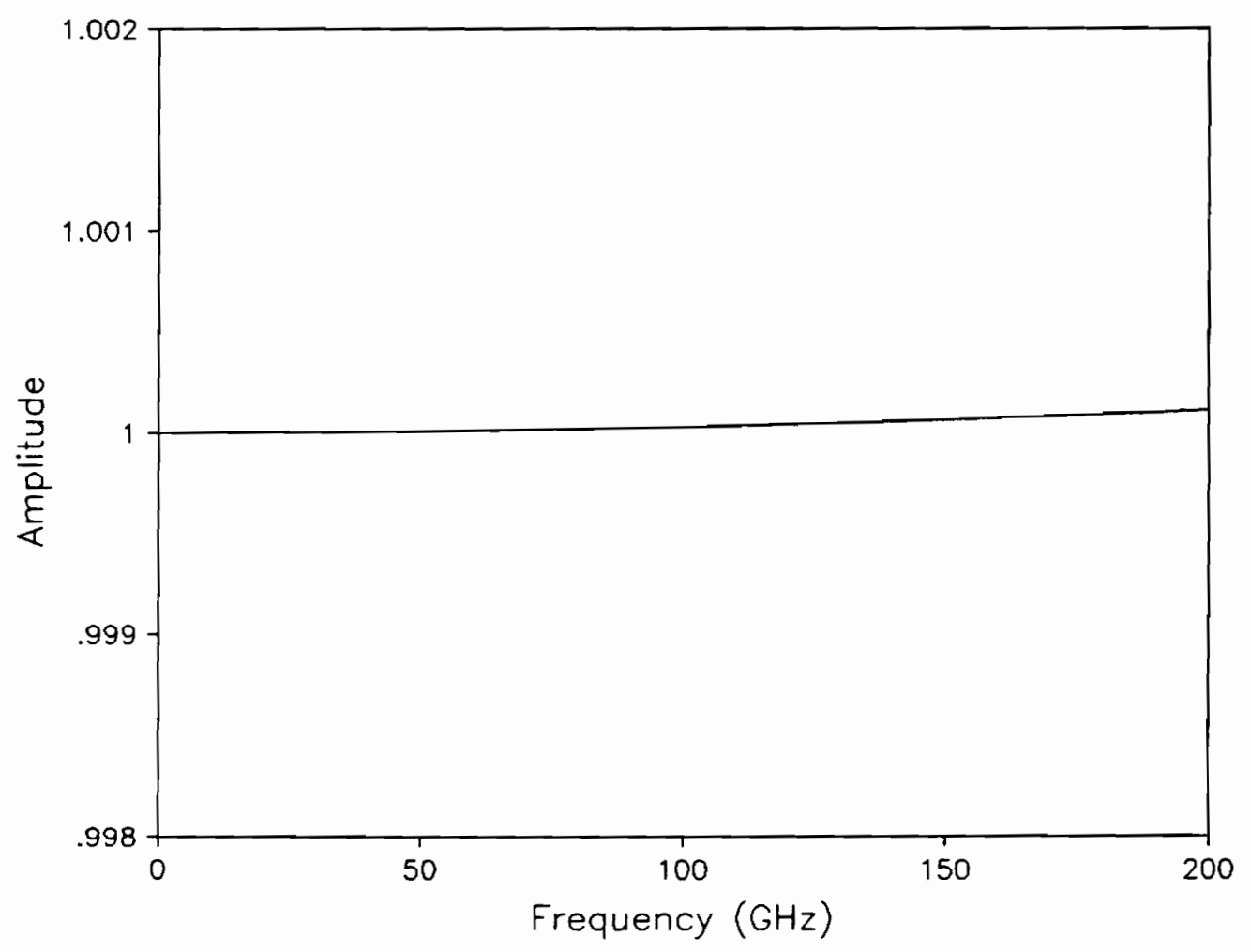

Figure 6.2.14 Results of the verification simulation: $S_{g 1} S_{1 r}$ amplitude versus frequency (shifting the open circuit TDR waveform 0.01 point forward and the short circuit TDR waveform 0.01 point backward). 


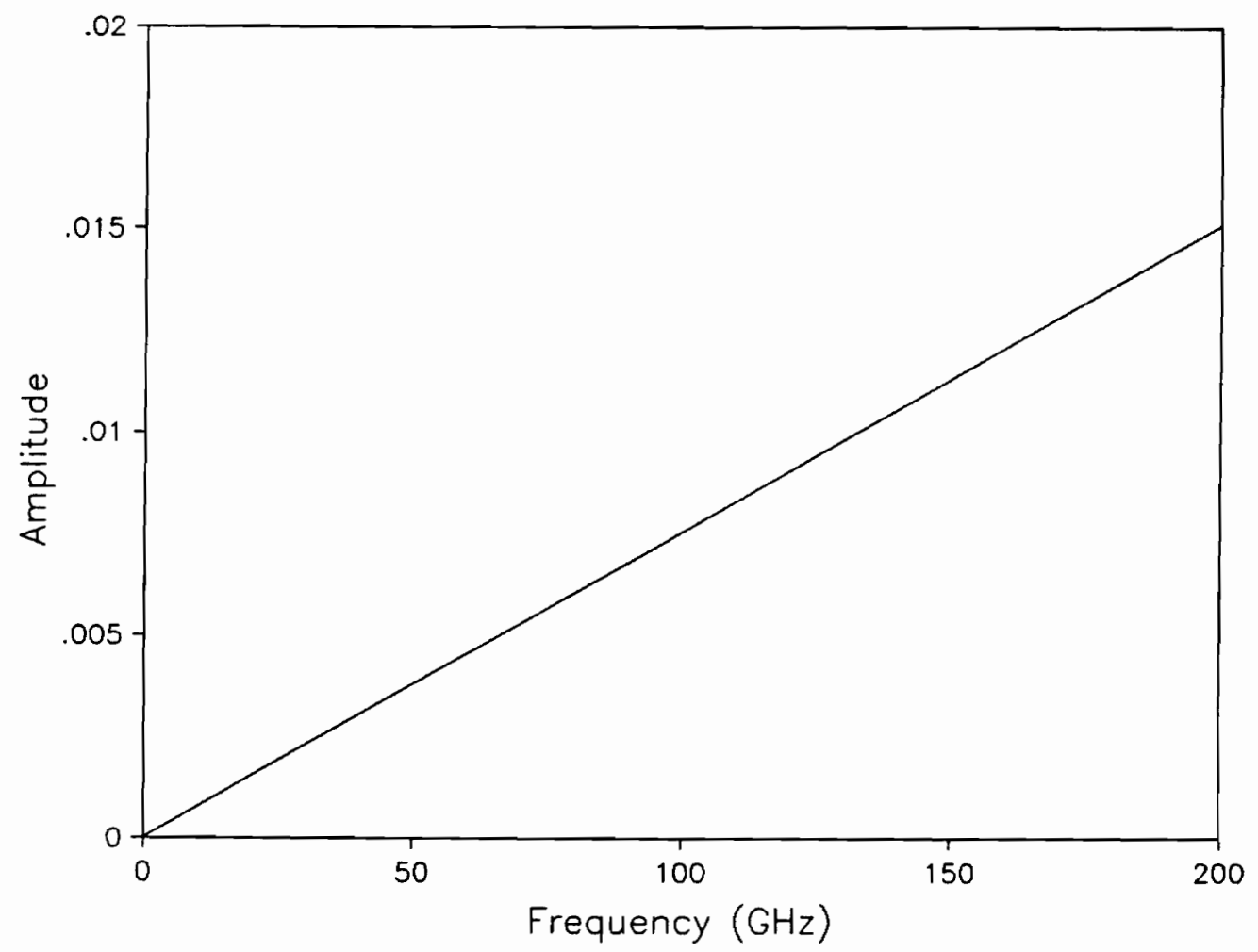

Figure 6.2.15 Results of the verification simulation: $S_{11}$ amplitude versus frequency (shifting the open circuit TDR waveform 0.01 point forward and the short circuit TDR waveform 0.01 point backward). 


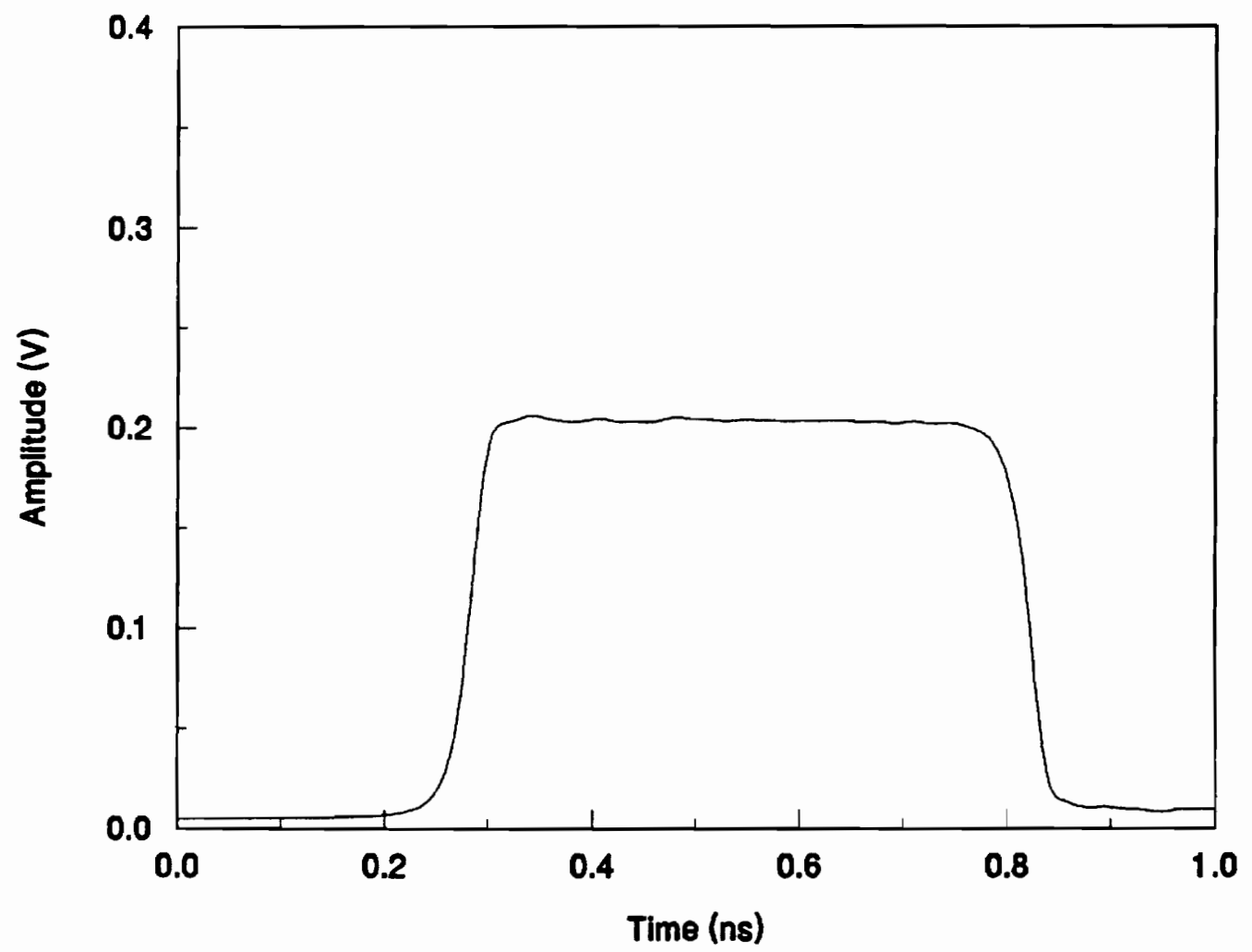

Figure 6.4.1 Acquired TDR response of the short circuit standard. 


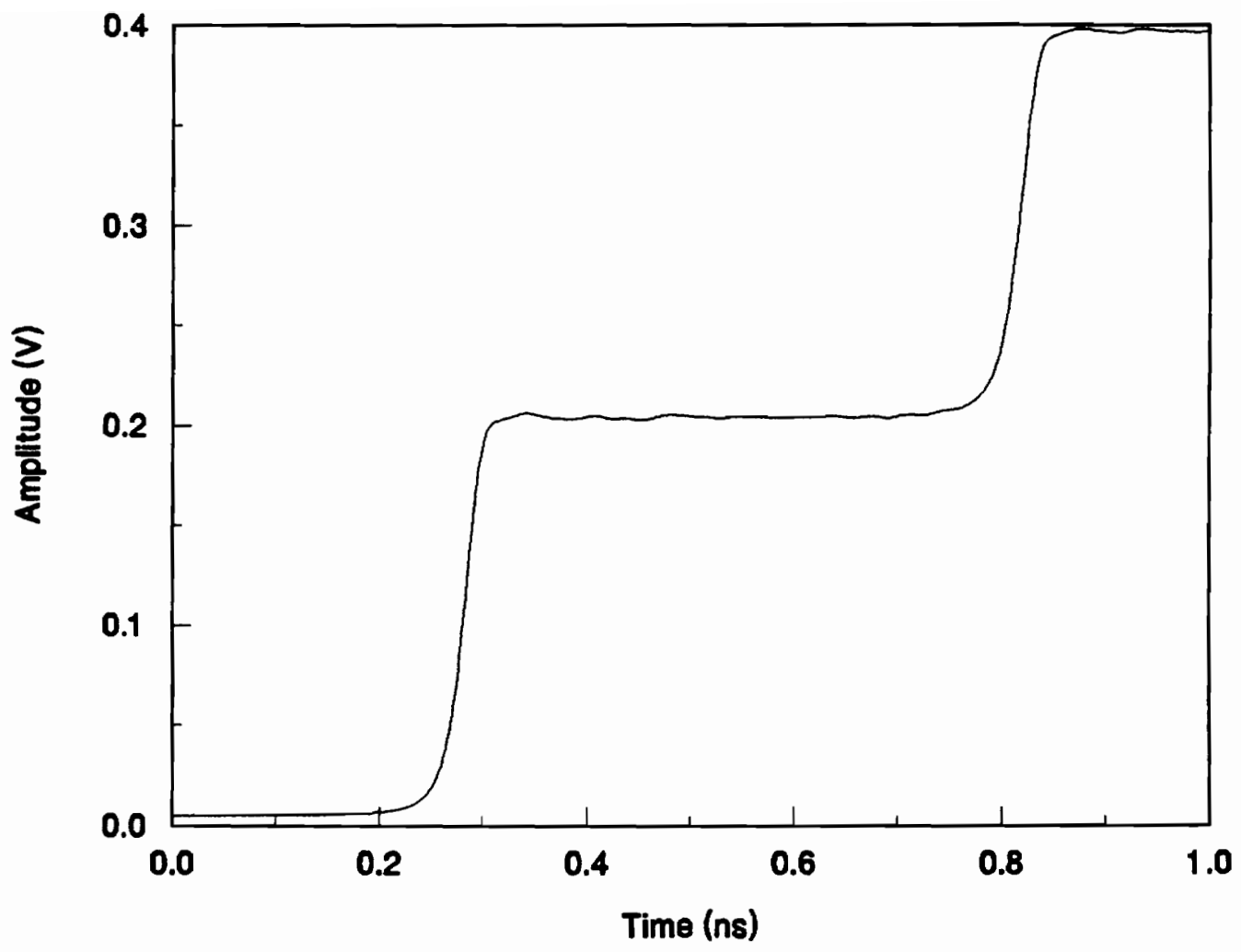

Figure 6.4.2 Acquired TDR response of the open circuit standard. 


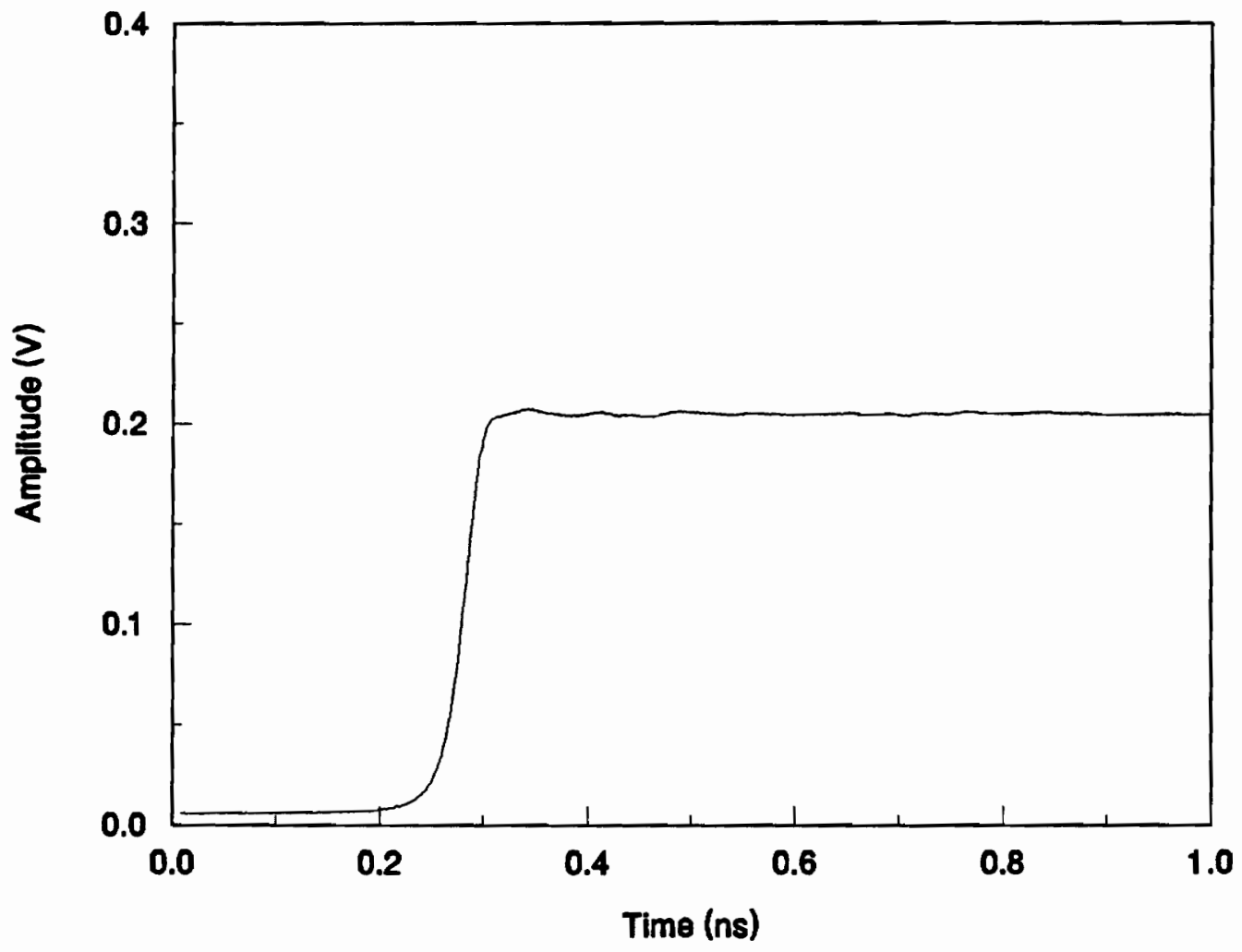

Figure 6.4.3 Acquired TDR response of the $50 \Omega$ coaxial air line. 


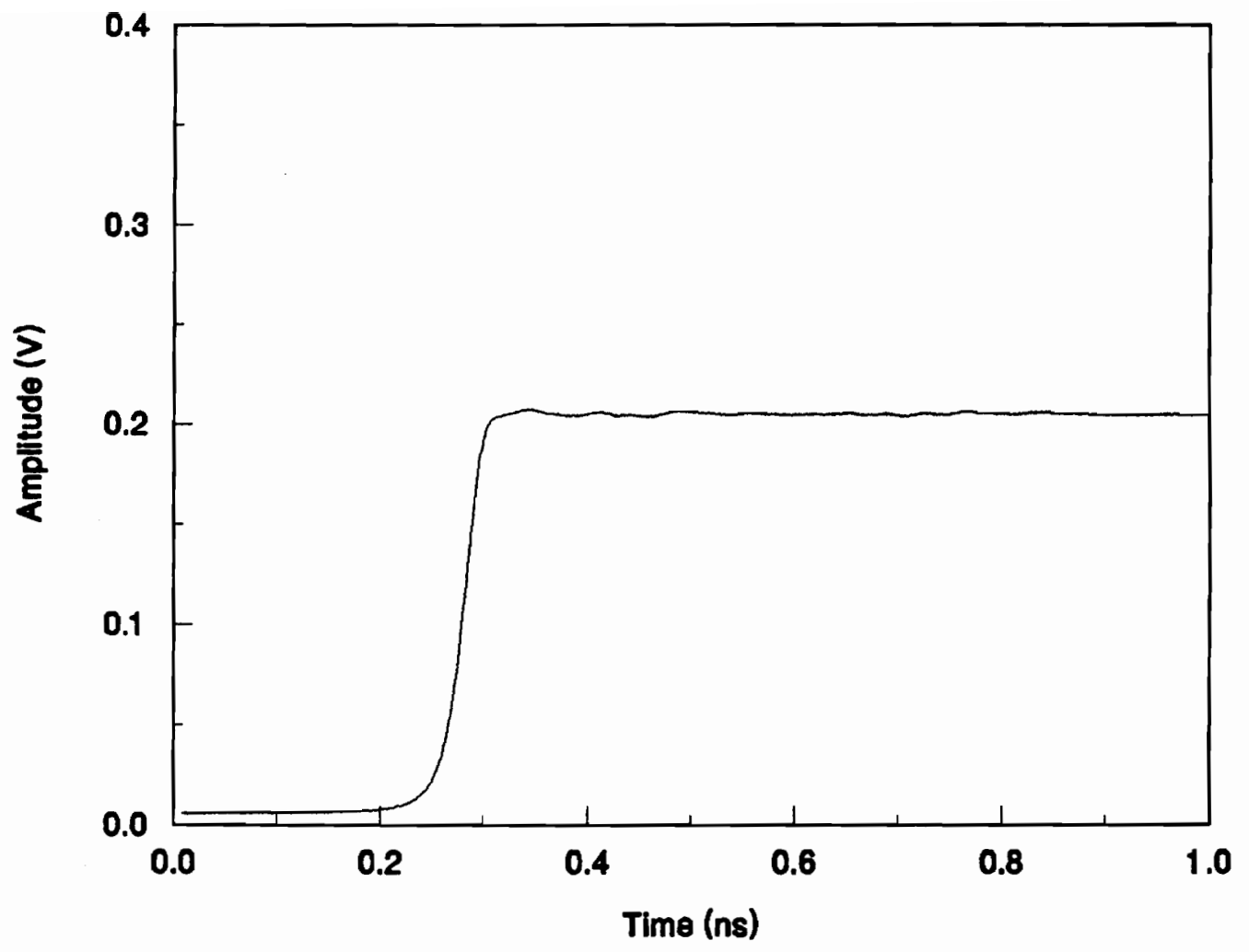

Figure 6.4.4 Acquired TDR response of the through connection. 


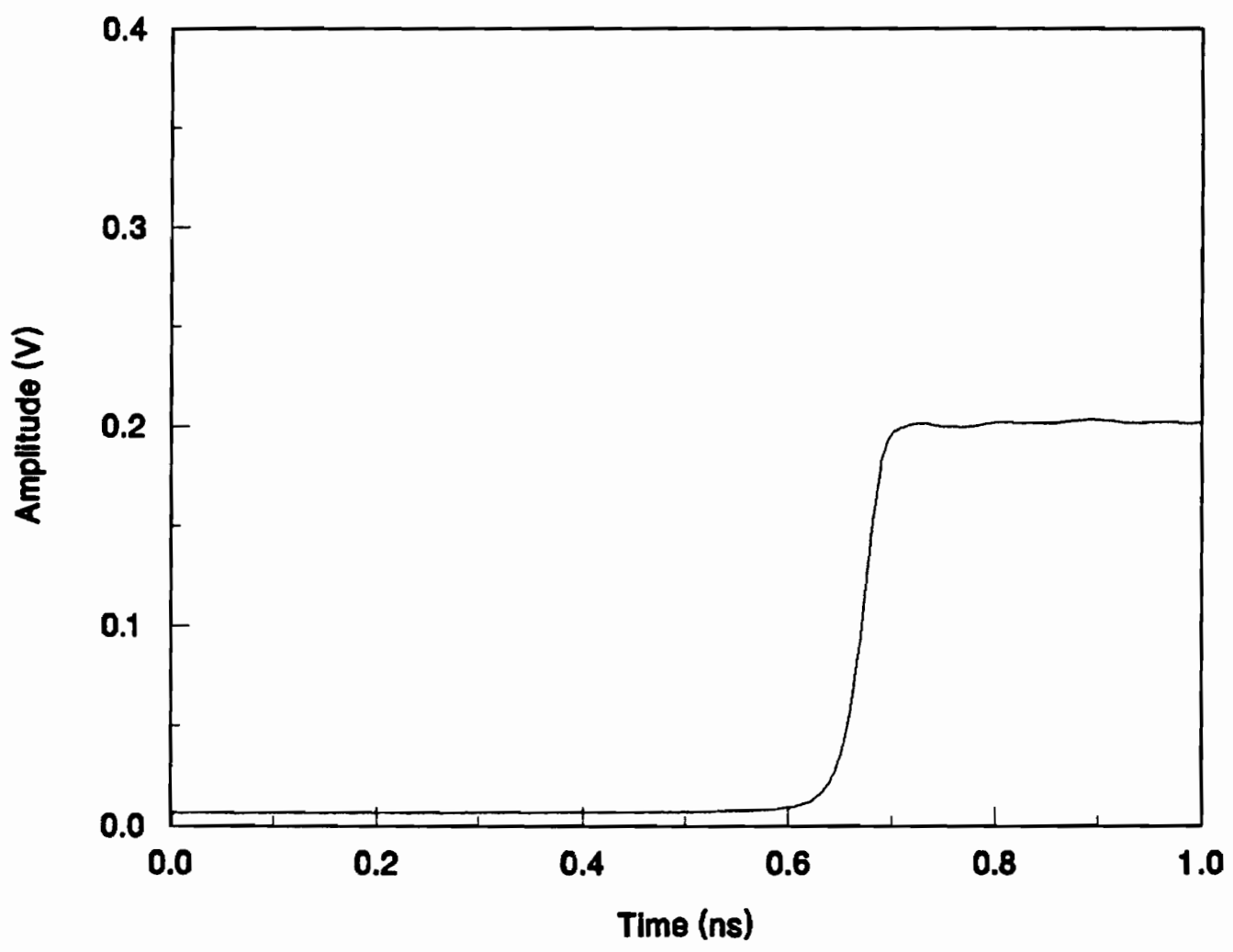

Figure 6.4.5 Acquired TDT response of the through connection. 


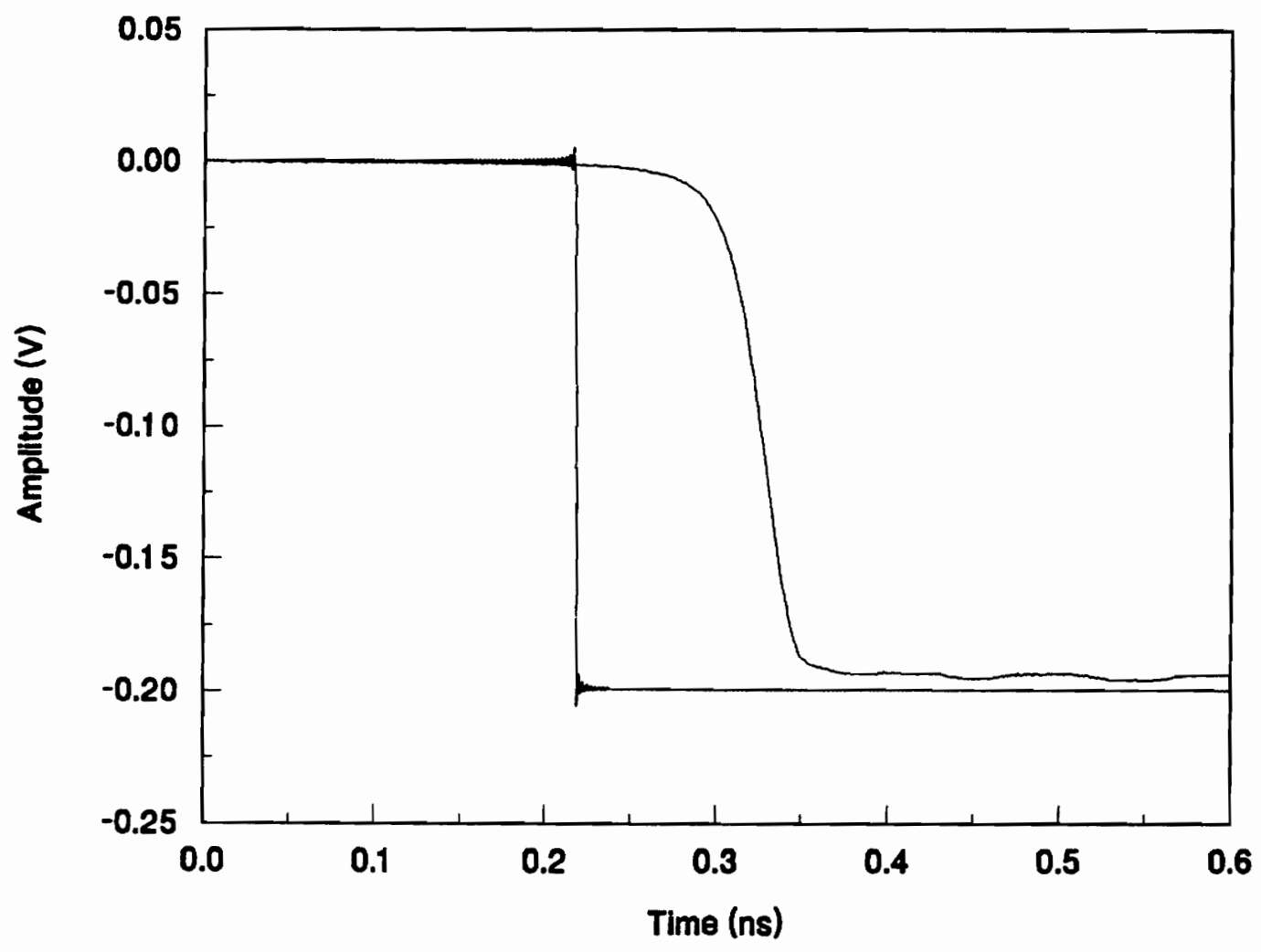

Figure 6.5.1 Acquired and de-embedded time domain TDR response for the short circuit standard. 


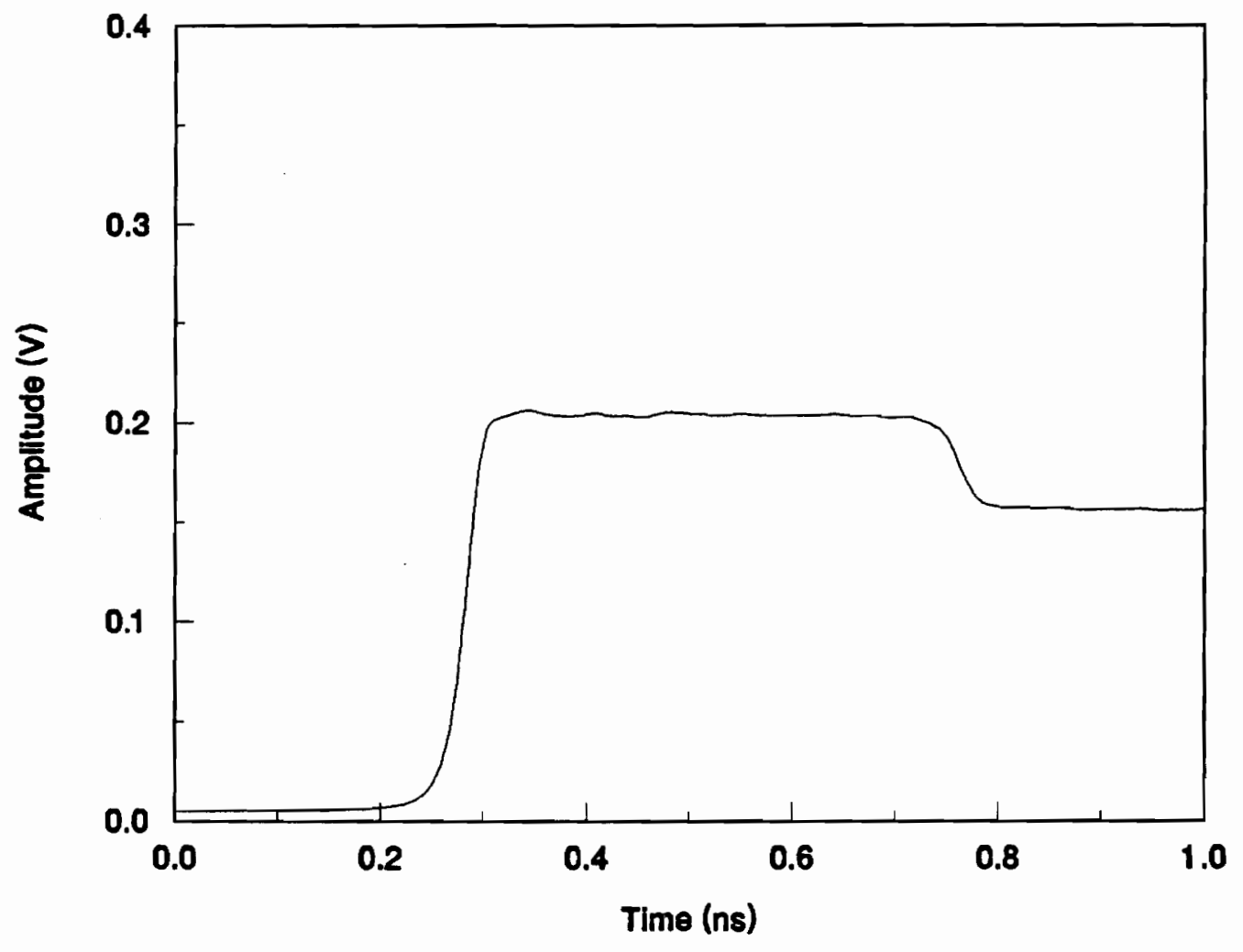

Figure 6.5.2 Acquired TDR response of the $30 \Omega$ coaxial air line. 


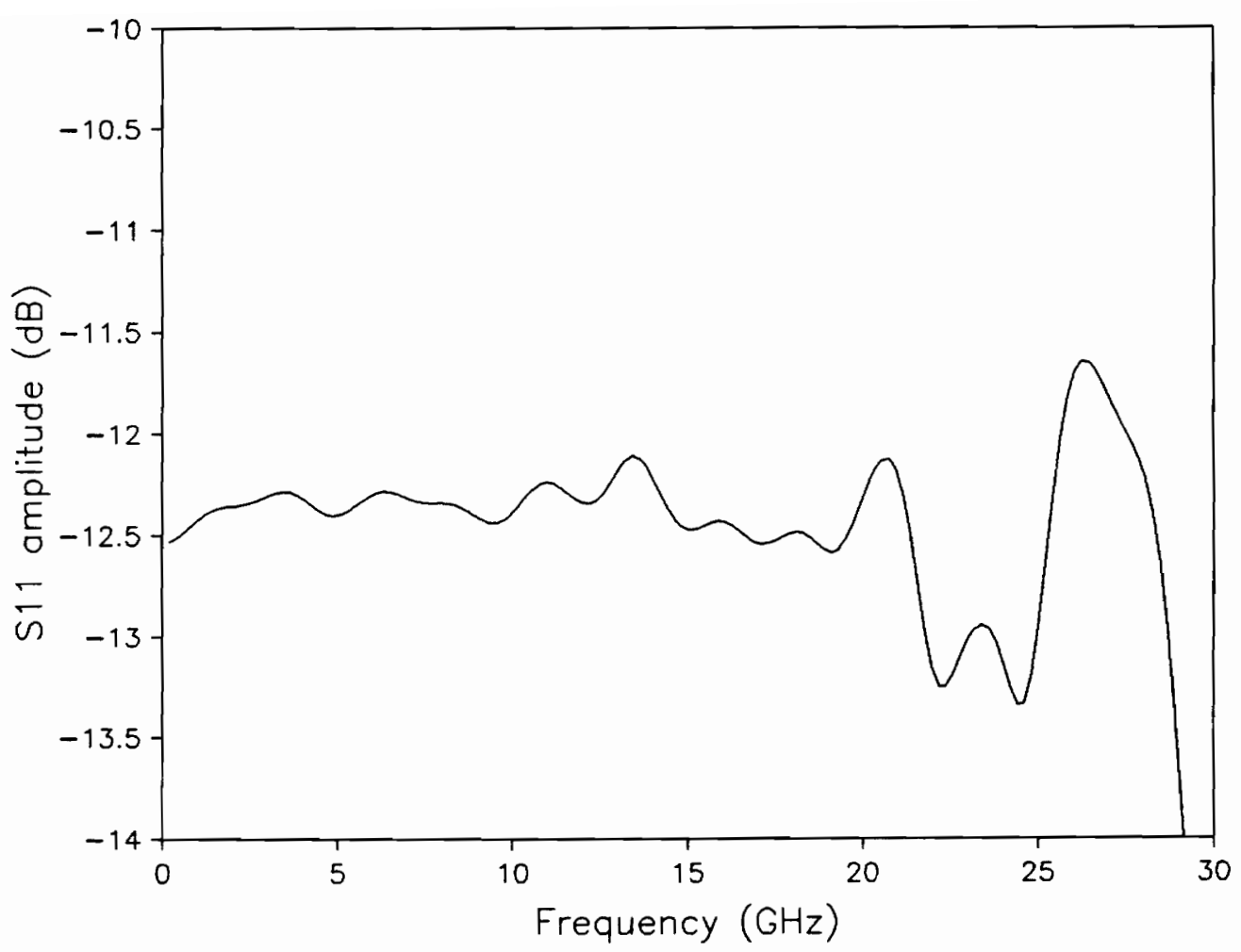

Figure 6.5.3 De-embedded $S_{11}$ amplitude response of the $30 \Omega$ coaxial air line. 


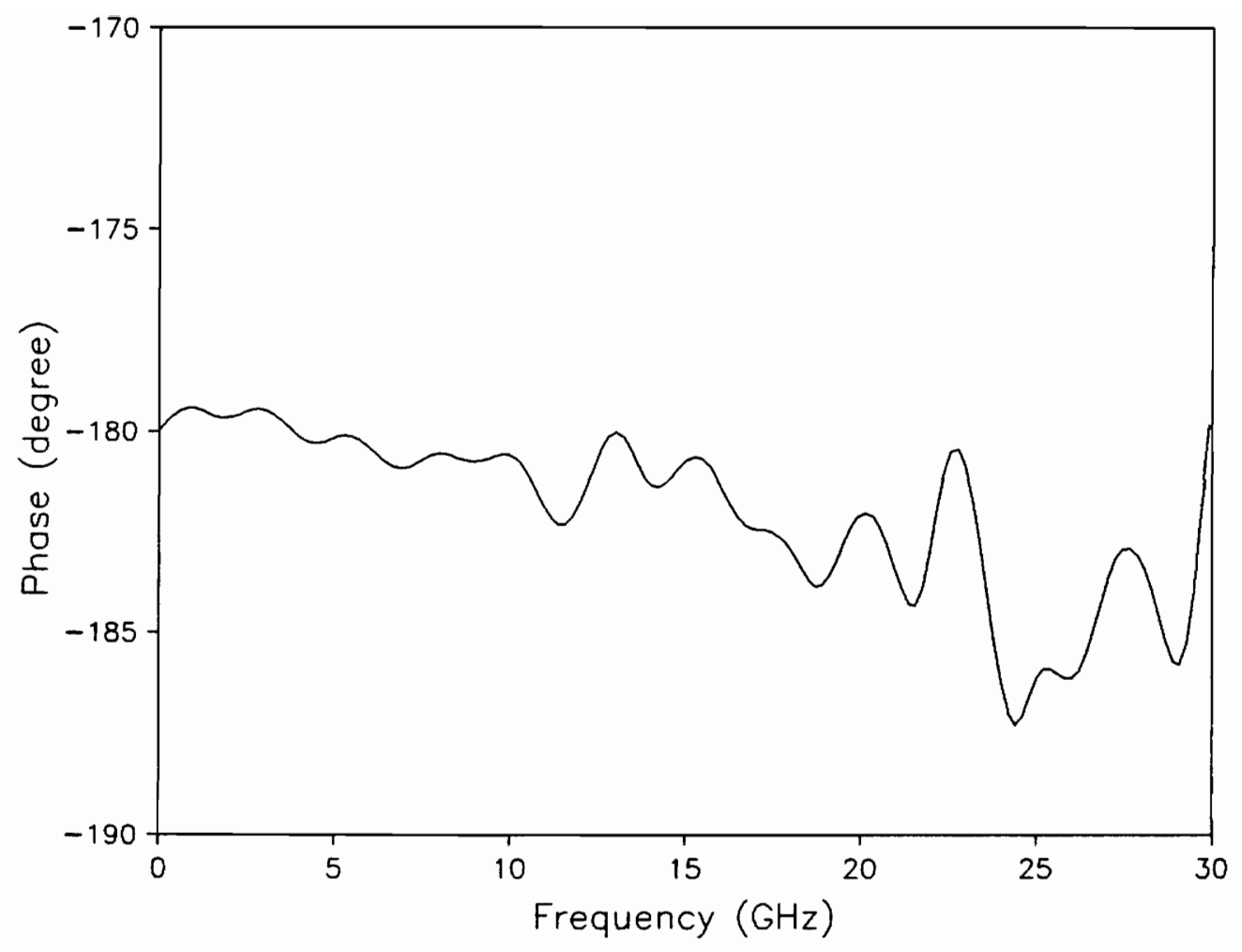

Figure 6.5.4 De-embedded $S_{11}$ phase response of the $30 \Omega$ coaxial air line. 


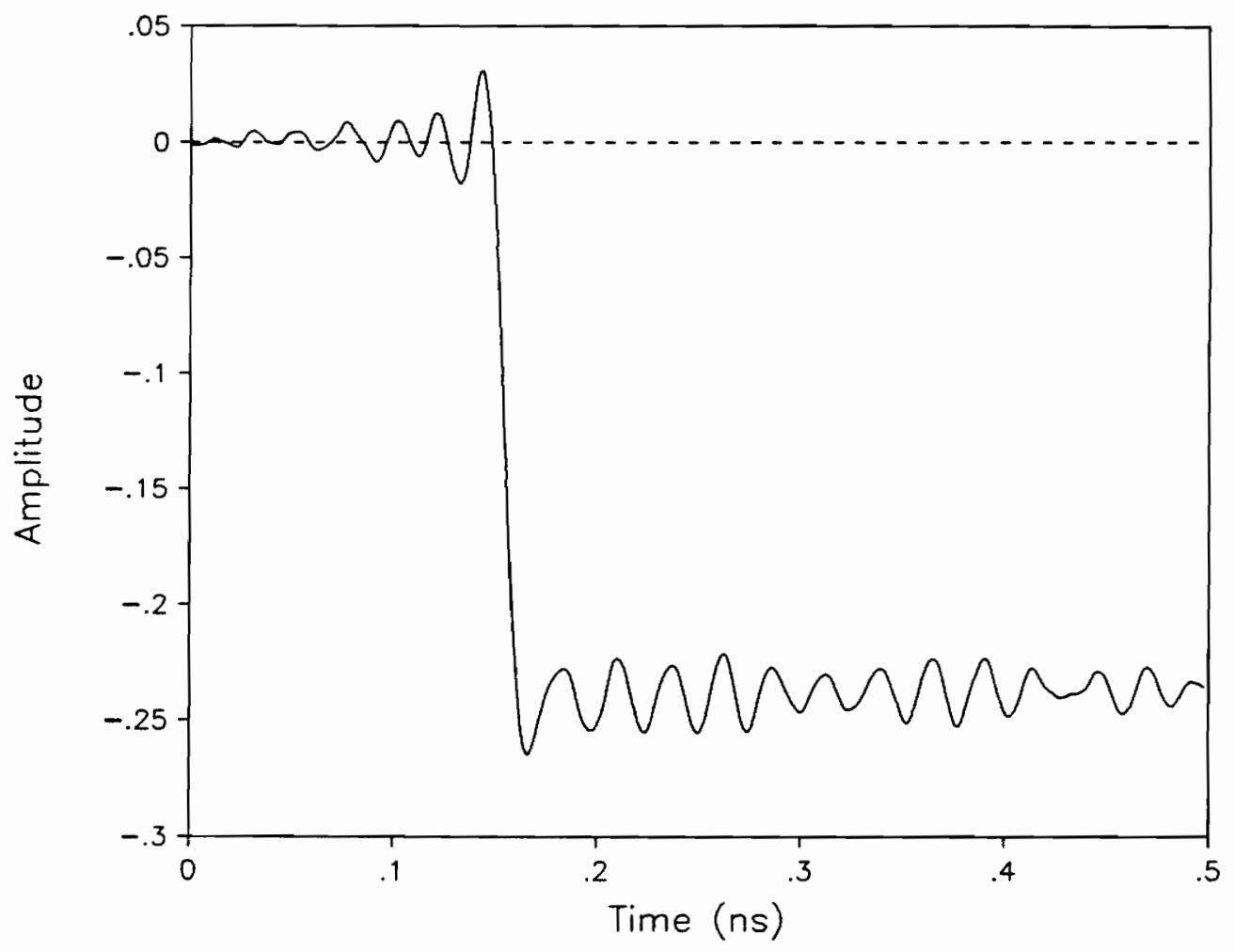

Figure 6.5.5 De-embedded step response of the $30 \Omega$ coaxial air line. 


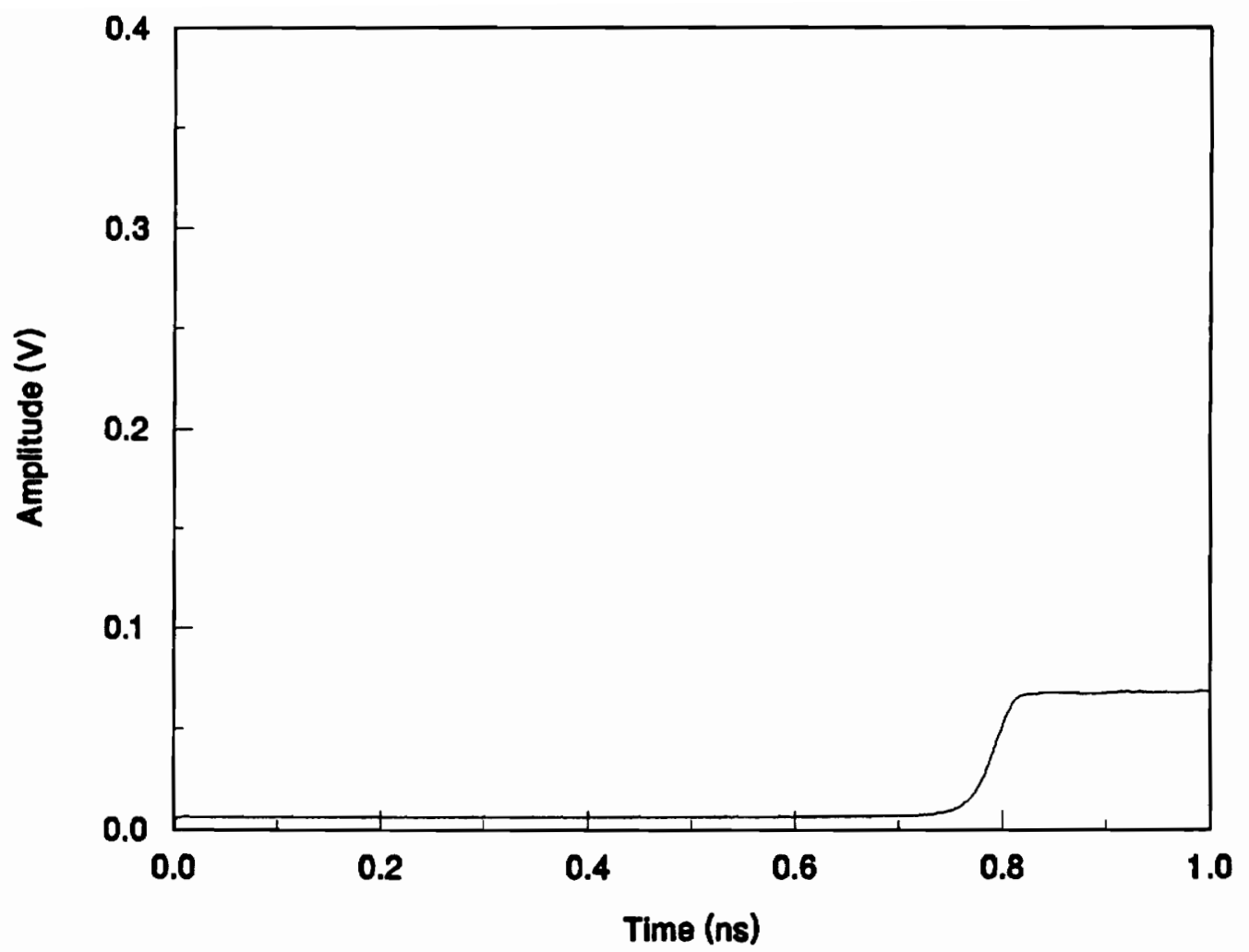

Figure 6.5.6 Acquired TDT response of the $10 \mathrm{~dB}$ attenuator. 


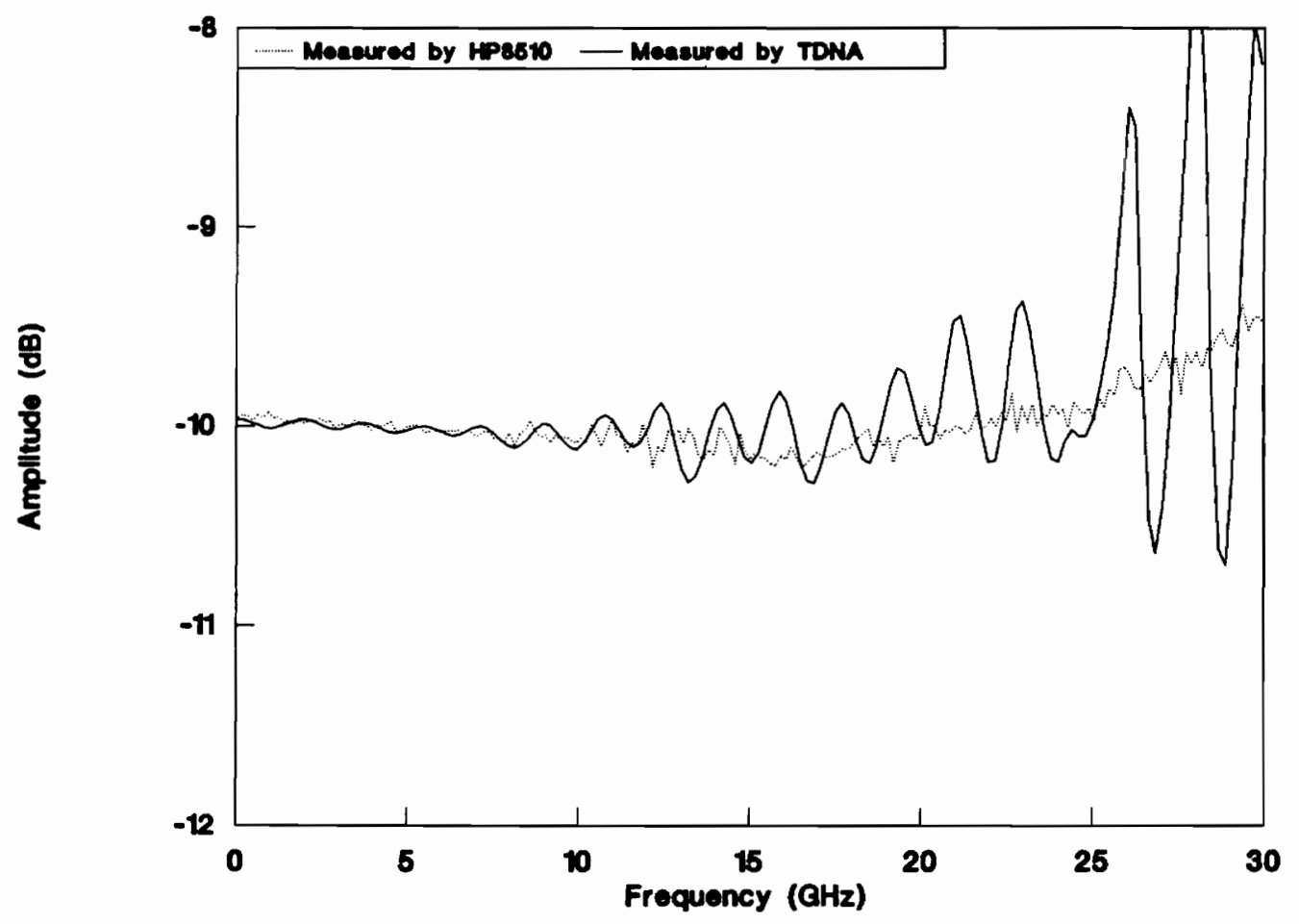

Figure 6.5.7 $S_{21}$ amplitude responses of the 10 $d B$ attenuator measured by time domain and frequency domain systems. 


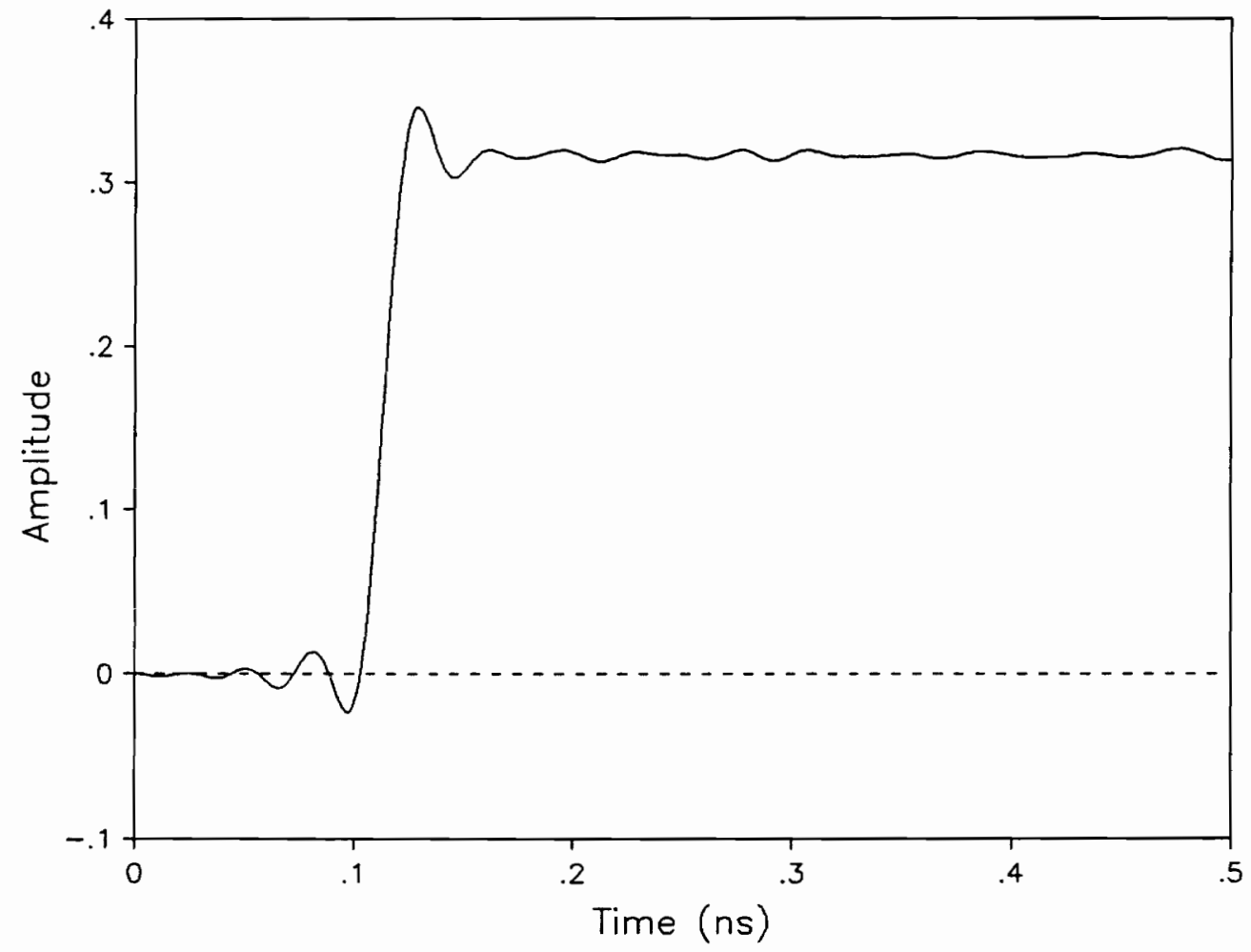

Figure 6.5.8 De-embedded step response of the $10 d B$ attenuator. 


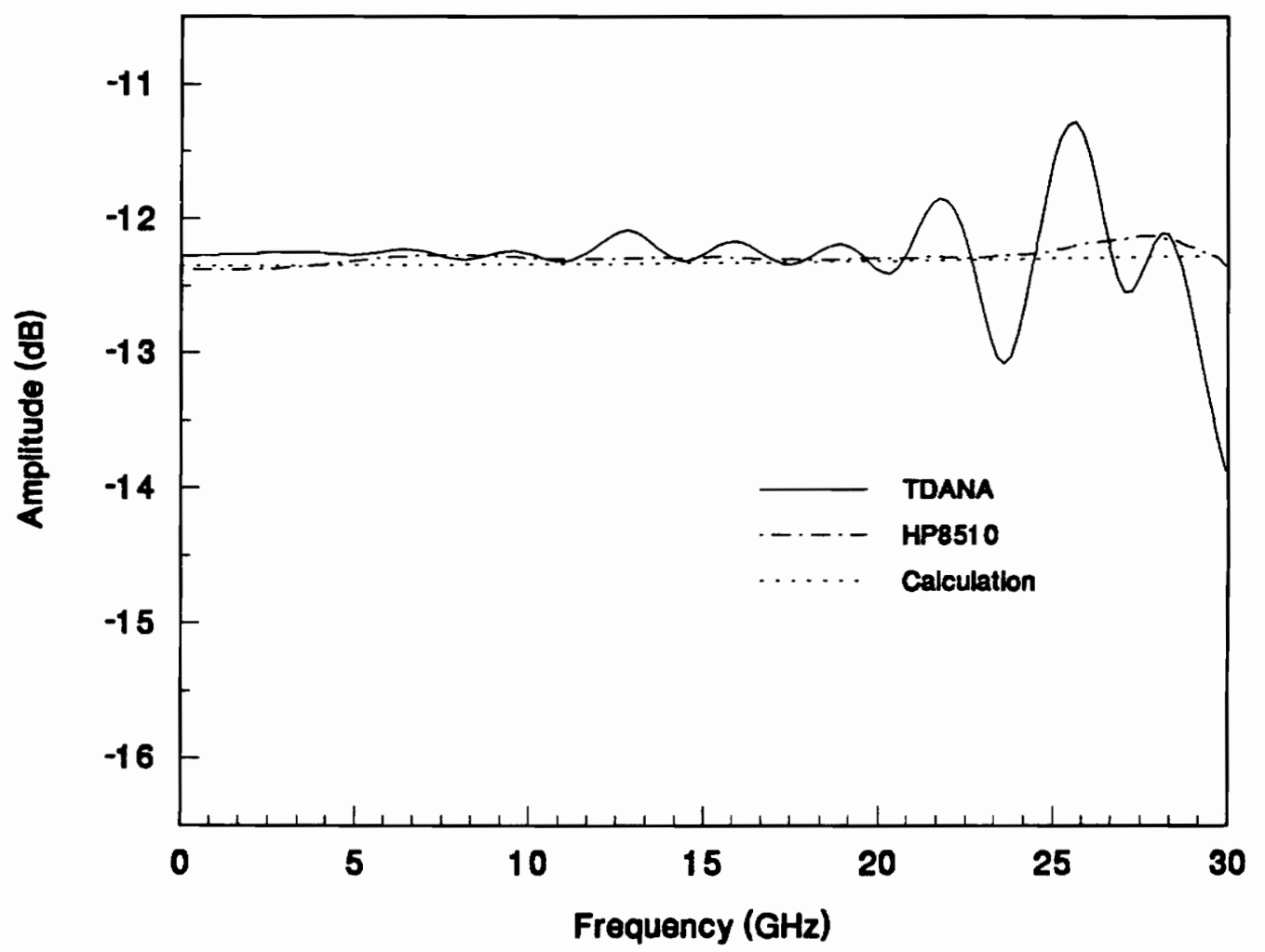

Figure 6.6.1 Comparison among the three sets of $S_{11}$ amplitude responses obtained by three different approaches for the $30 \Omega$ coaxial line. 


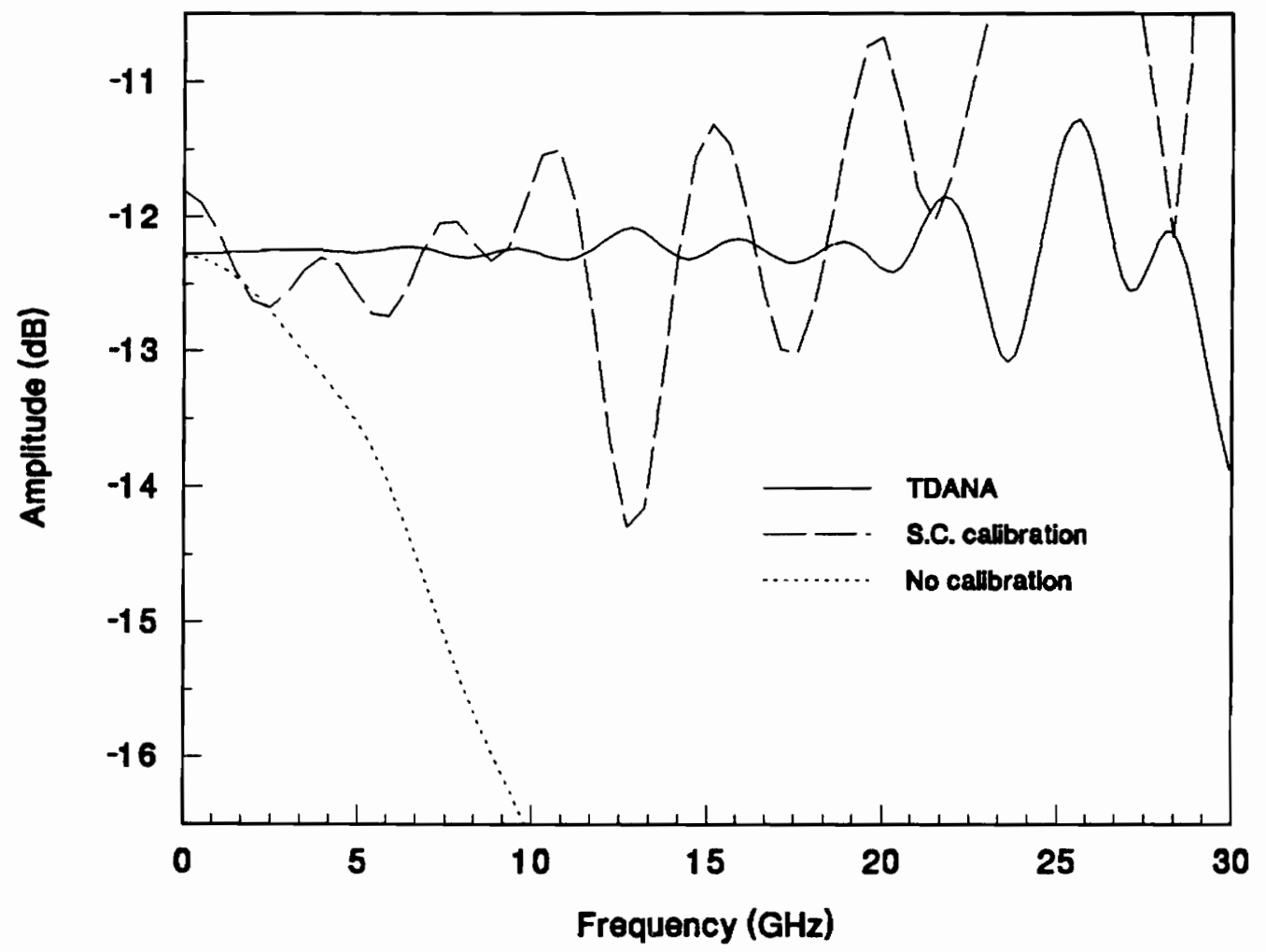

Figure 6.6.2 Comparison among the three sets of $S_{11}$ amplitude responses obtained with no calibration, simple short circuit calibration and the complete calibration. 


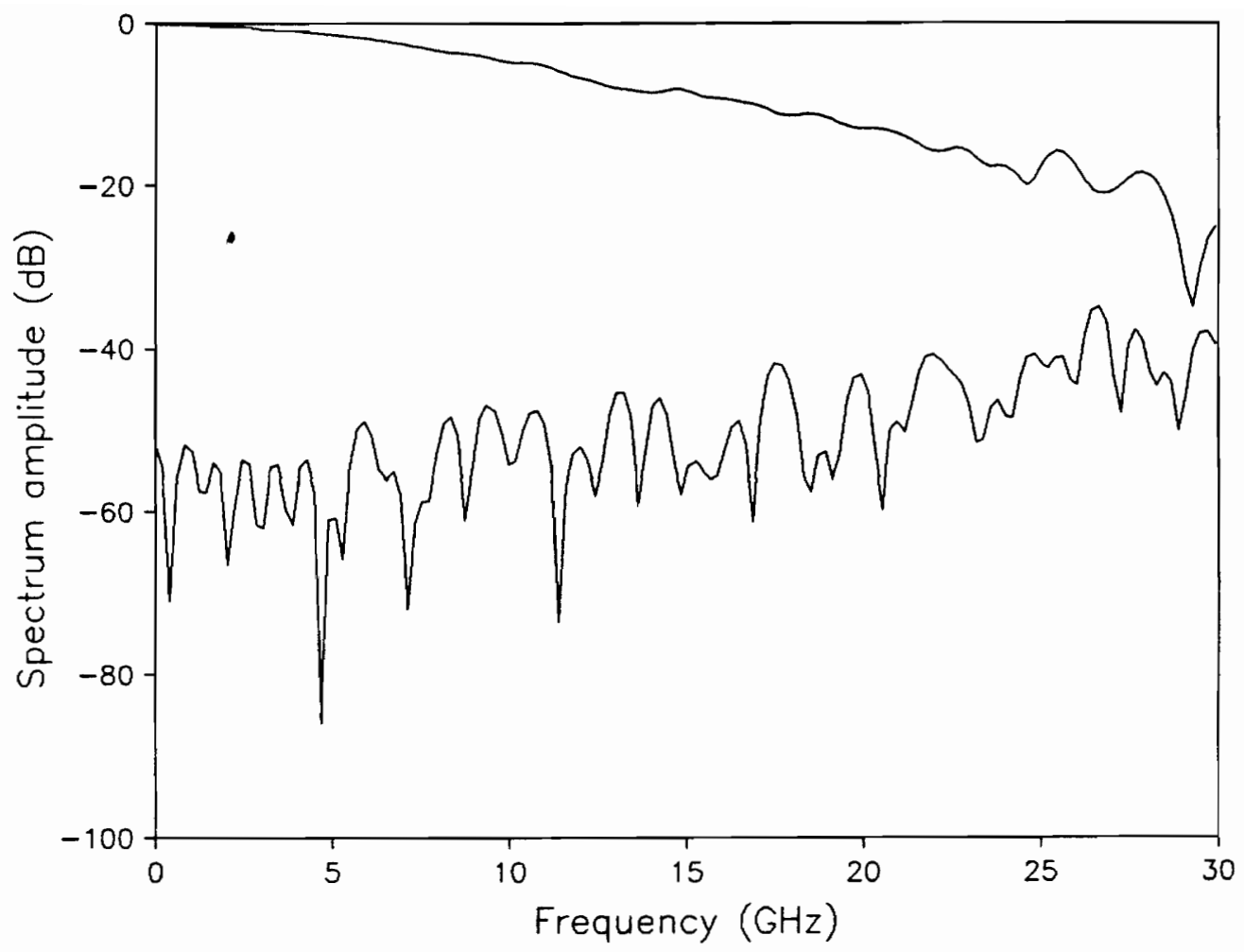

Figure 6.6.3 The normalized signal spectrum amplitude and background noise spectrum amplitude of the system. 


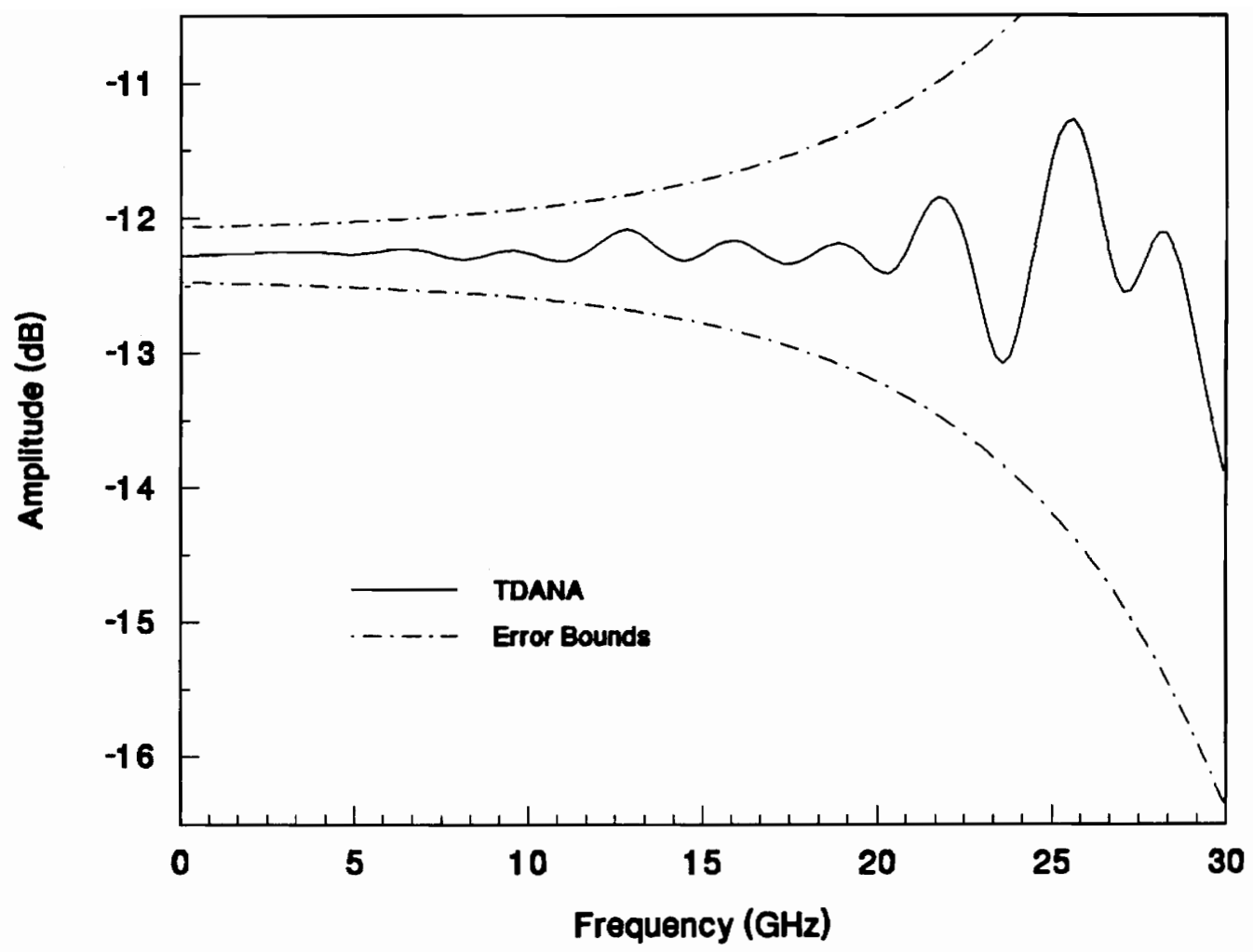

Figure 6.6.4 Calculated error bounds for the $S_{11}$ amplitude response of the $30 \Omega$ coaxial line. Curve fitting in the Axum ${ }^{\circledR}$ software is used to obtain smooth error bound curves. 


\section{CHAPTER 7}

\section{SUMMARY AND CONCLUSION}

\subsection{SUMMARY}

In this research, a generic model for TDNA measurement systems has been established, shown in Fig. 2.3.2. A set of three time domain calibration standards has been developed and used to calibrate TDNA systems. The set of calibration equations developed in Chapter 2 is used to evaluate the system model parameters and summarized here.

$$
\begin{aligned}
& S_{11}=\frac{W_{r 1}\left(\Gamma_{s}-\Gamma_{2}\right)+W_{r 2}\left(\Gamma_{1}-\Gamma_{g}\right)+W_{r g}\left(\Gamma_{2}-\Gamma_{1}\right)}{\Gamma_{1} W_{r 1}\left(\Gamma_{s}-\Gamma_{2}\right)+\Gamma_{2} W_{r 2}\left(\Gamma_{1}-\Gamma_{s}\right)+\Gamma_{s} W_{r g}\left(\Gamma_{2}-\Gamma_{1}\right)}, \\
& S_{g r}=\frac{\left(W_{r 1} \Gamma_{2}-W_{r 2} \Gamma_{1}\right)-S_{11} \Gamma_{1} \Gamma_{2}\left(W_{r 1}-W_{r 2}\right)}{E_{g}\left(\Gamma_{2}-\Gamma_{1}\right)}, \\
& S_{g 1} S_{1 r}=\left(\frac{W_{r 1}}{E_{g}}-S_{g r}\right)\left(\frac{1}{\Gamma_{1}}-S_{11}\right), \\
& S_{11} S_{22}=\frac{W_{r 4}}{E_{g}}-S_{g r} \\
& S_{g 1} S_{1 r}+S_{11}\left(\frac{W_{r 4}}{E_{g}}-S_{g r}\right)
\end{aligned}
$$

and 
$S_{g 1} S_{2 t}=\frac{W_{t 4}}{E_{g}}\left(1-S_{11} S_{22}\right)$

Using the set of three calibration standards $\left(\Gamma_{1}, \Gamma_{2}\right.$, and $\left.\Gamma_{3}\right)$ enables us to acquire three TDR response waveforms $\left(W_{r 1}, W_{r 2}\right.$, and $\left.W_{r s}\right)$. Connecting channel 1 directly to channel 2 enables us to obtain the other two waveforms, $W_{r 4}$ acquired from channel 1 and $W_{44}$ from channel 2. Substituting these known parameters into the above five equations, system model parameters can be obtained.

Then, these obtained model parameters can be used to de-embedding the system effects. From Chapter 5, the two de-embedding equations are

$\Gamma_{d u t}=\frac{\frac{W_{d u t}}{E_{g}}-S_{g r}}{\left(\frac{W_{d z t}}{E_{g}}-S_{g r}\right) S_{11}+S_{g 1} S_{1 r}}$ for TDR

and

$S_{21 d u t}=\frac{W_{t 1}\left(1-S_{11} S_{11 d u t}-S_{22} S_{22 d u t}\right)}{S_{g 1} S_{t 2}}$ for TDT.

Assuming measurements with equal accuracy, the possible errors for determining the device under test are

$\left|\Delta \Gamma_{d \mathbf{x t}}\right|=5|\Delta W|$

and

$\left|\Delta S_{21 d u t}\right|=2|\Delta W|$

Chapter 7. Summary and Conclusion 
These two equations give us error bounds of the measurement uncertainties.

\subsection{CONCLUSIONS}

Compared to the time domain and frequency domain results, a fairly good accuracy has been achieved. This verifies the validity of the calibration standards developed and equivalent model for the time domain network analyzer system. Theoretically, the calibration procedure can be used to obtain an ideal step or impulse response of the device under test. Practically, there are two main limiting factors to the bandwidth of the calibrated system. These factors are: (1) noise (system background noise, measurement errors, computational errors, etc.) and (2) high order modes. For a $3.5 \mathrm{~mm}$ system, although theoretical calculation predicts that the first high-order mode appears above $30 \mathrm{GHz}$, the manufacturers suggest using them under $26.5 \mathrm{GHz}$ due to the tolerance of mechanical processing and manufacturing. Models accounting for high-order modes can be developed. Such model would be highly dependent on the source polarization and the nature of discontinuities in both the system and the DUT and would not be universal for a general DUT. This interprets that small discontinuities of the device under test could dramatically change the measurement result. For this reason, the dominant mode model is preferred.

In conclusion, the calibration extended the bandwidth of the time domain network analyzer more than 2.5 times. The experimental verification has demonstrated the validity and accuracy of the technique. Due to the generic nature of the system model, this technique can be used for other time domain systems. 


\section{APPENDIX}

\section{A. RELATION OF ORIGINAL AND SIMPLIFIED MODEL PARAMETERS}

The relation of the parameters between the original and simplified models is as follows.

$S_{g r}=\frac{\frac{S_{d 1 t} S_{b 21} S_{p t}}{1-S_{d 1} S_{b 22}}}{1-\frac{S_{p r} S_{b 21}\left(S_{b 12}+S_{d 1 r}\right)}{\left(1-S_{d i r} S_{b 22}\right)\left[1-S_{p r}\left(S_{b 11}+S_{a 11}\right)\right]}}$

$S_{g 1}=\frac{S_{p t} S_{a 21}}{1-\frac{S_{p r} S_{b 21}\left(S_{b 12}+S_{d 1 r}\right)}{\left(1-S_{d i r} S_{b 22}\right)\left[1-S_{p r}\left(S_{b 11}+S_{a 11}\right)\right]}}$

$S_{1 r}=\frac{\frac{S_{a 12} S_{p r} S_{b 21} S_{d 1 t}}{\left(1-S_{d i r} S_{b 22}\right)\left[1-S_{p r}\left(S_{b 11}+S_{a 11}\right)\right]}}{1-\frac{S_{p r} S_{b 21}\left(S_{b 12}+S_{d 1 r}\right)}{\left(1-S_{d i r} S_{b 22}\right)\left[1-S_{p r}\left(S_{b 11}+S_{a 11}\right)\right]}}$

$S_{11}=\frac{\frac{S_{a 12} S_{p r} S_{a 21}}{1-S_{p r}\left(S_{b 11}+S_{a 11}\right)}}{1-\frac{S_{p r} S_{b 21}\left(S_{b 12}+S_{d 1 r}\right)}{\left(1-S_{d i r} S_{b 22}\right)\left[1-S_{p r}\left(S_{b 11}+S_{a 11}\right)\right]}}+S_{a 22}$

$S_{2 t}=\frac{S_{d 2 t S_{c 21}}}{1-S_{c 22} S_{d 2 r}}$

Appendix 


$$
S_{2 r}=S_{c 11}+\frac{S_{c 21} S_{c 12} S_{d 2 r}}{1-S_{c 22} S_{d 2 r}}
$$

\section{B. COAXIAL OPEN CIRCUIT DISCONTINUITY}

Figure 3.1.2 shows a typical commercial available open circuit standard. The shielded open end is used to prevent open end radiation. If we neglecting the conducting wall loss, at the terminal plane, the equivalent circuit is shown in Fig. 3.1.2. Now, we use variational approach to derive the formula to calculate the equivalent capacitance $C$ [28]. The procedures are as follows.

\section{B.1 Find mode functions}

From Fig. 3.1.2, an open standard consists of two sections: the coaxial line section and the circular waveguide section shorted at the end. We consider them separately as follows.

\section{A.) Coaxial line section}

Due to the symmetrical structure of the standard, the possible modes in the coaxial section are TEM mode and TM modes.

I. TEM mode

The eigenfunction $\Phi(\rho, \phi)$ satisfies

$$
\nabla^{2} \Phi(\rho, \phi)=0 .
$$


The solution, which is independent of $\phi$, is

$\Phi(\rho)=A \ln (\rho)+B$

where $A$ and $B$ are constants determined by the boundary conditions. The electric and magnetic field components are

$E_{\rho}=\frac{A}{\rho} e^{ \pm j k z}=\Phi_{0}(\rho) e^{ \pm j k z}$

$\sqrt{\frac{\mu}{\epsilon}} H_{\phi}=\frac{A}{\rho} e^{ \pm j k z}$

where $\Phi_{0}(\rho)=\frac{A}{\rho}$ and $k=\omega \sqrt{\mu \epsilon}$

II. TM modes

The eigenfunctions $\Phi_{n}(\rho)$ satisfy

$\nabla^{2} \Phi_{n}(\rho, \phi)+\gamma_{n}^{2} \Phi_{n}(\rho, \phi)=0$

with boundary conditions $\left.\Phi_{n}(\rho)\right|_{\rho=a, b}=0$. The solutions, which are independent of $\phi$ are

$\Phi_{n}(\rho)=\frac{\sqrt{\pi}}{2} \frac{J_{0}\left(\gamma_{n} \rho\right) N_{0}\left(\gamma_{n} b\right)-N_{0}\left(\gamma_{n} \rho\right) J_{0}\left(\gamma_{n} b\right)}{\left[\frac{J_{0}^{2}\left(\gamma_{n} b\right)}{J_{0}^{2}\left(\gamma_{n} a\right)}-1\right]^{1 / 2}}, \quad n=1,2, \ldots$

The eigenvalues $\gamma_{n}$ are determined form the equation $\Phi_{n}(a)=0$. 
The transverse components of the field follow from

$$
\begin{aligned}
& \vec{E}_{t n}= \pm j h_{n}\left(\nabla \Phi_{n}\right) e^{ \pm j h_{n} z}= \pm \phi_{n} e^{ \pm j h_{n} z}, \quad \phi_{n}=j h_{n} \nabla \Phi_{n} \\
& \pm z_{n} \vec{H}_{t n}=\widehat{e}_{z} \times \vec{E}_{t n}
\end{aligned}
$$

and are as follows

$E_{\rho n}=j h_{n} \gamma_{n} \Phi_{n}^{\prime}(\rho) e^{ \pm j h_{n} z}= \pm \phi_{n}(\rho) e^{ \pm j h_{n} z}$

$H_{\phi n}=\frac{1}{z_{n}} E_{\rho n}$,

where $z_{n}=\frac{h_{n}}{\omega \epsilon}$ and $h_{n}=\sqrt{k^{2}-\gamma_{n}^{2}}$.

B.) Circular waveguide short at the end

The eigenfunctions $\Psi_{n}(\rho, \phi)$ satisfy

$$
\nabla^{2} \Psi_{n}(\rho, \phi)+\gamma_{n}^{\prime}{ }^{2} \Psi_{n}(\rho, \phi)=0
$$

where the boundary condition is $\Psi_{n}=0$ for $\rho=a$. The solutions, which are independent of $\phi$ are

$\Psi_{n}(\rho)=\frac{1}{\sqrt{\pi} \gamma_{n}^{\prime} a} \frac{J_{0}\left(\gamma_{n} \rho\right)}{J_{1}\left(\gamma_{n} a\right)}, \quad \quad n=1,2, \ldots$

The eigenvalues $\gamma_{n}^{\prime}$ are determined form the equation $\mathrm{J}_{0}\left({\gamma^{\prime}}_{n} a\right)=0$. 
The solutions for fields are

$E_{\rho n}=j h_{n}^{\prime} \nabla \Psi_{n}(\rho) \sin \left[j h_{n}^{\prime}(z-l)\right]=\psi_{n}(\rho) \sin \left[j h_{n}^{\prime}(z-l)\right]$

$H_{\phi n}=\frac{1}{z_{n}} E_{\rho n}$

where $z_{n}^{\prime}=\frac{h_{n}^{\prime}}{\omega \epsilon}$ and $h_{n}^{\prime}=\sqrt{k^{2}-\gamma_{n}^{\prime}{ }^{2}}$

\section{B.2 Constructing the field expressions}

Assume an incident TEM mode wave propagating in the coaxial line to the $z$ direction. Due to the structure symmetry, only TM modes, independent of $\phi$, can be excited by the discontinuity at $z=0$. The mode expressions for the transverse components of the electric and magnetic fields are

$E_{\rho}=a_{0}\left(e^{j k z}+R e^{-j k z}\right) \phi_{0}(\rho)+\sum_{n=1}^{\infty} a_{n} \phi_{n}(\rho) e^{-j h_{n} z}$

$H_{\phi}=a_{0}\left(e^{j k z}-R e^{-j k z}\right) y_{0} \phi_{0}(\rho)-\sum_{n=1}^{\infty} a_{n} y_{n} \phi_{n}(\rho) e^{-j h_{n} z}$

for $z<0$ and $b \leq \rho \leq a$. In equations (B.2.1) and (B.2.2), $R=\frac{b_{0}}{a_{0}}$, and $b_{0}$ and $a_{0}$ are the amplitudes of the reflected and incident waves, respectively.

In the region $z>0$ and $0 \leq \rho \leq a$, the fields are

$E_{\rho}=\sum_{n=1}^{\infty} b_{n} \psi_{n}(\rho) \sin \left[h_{n}^{\prime}(z-l)\right]$, 
$H_{\phi}=\sum_{n=1}^{\infty} b_{n} y_{n}^{\prime} \psi_{n}(\rho) \sin \left[h_{n}^{\prime}(z-l)\right]$

The transverse field components must be continuous across the boundary at $z=0$, requiring

$$
\begin{array}{ll}
E_{t}=a_{0}(1+R) \phi_{0}(\rho)+\sum_{n=1}^{\infty} a_{n} \phi_{n}(\rho)=\sum_{n=1}^{\infty} b_{n} \psi_{n}(\rho) \sin \left(h_{n}^{\prime} l\right) & b \leq \rho \leq a \\
H_{t}=a_{0}(1-R) y_{0} \phi_{0}(\rho)-\sum_{n=1}^{\infty} a_{n} y_{n} \phi_{n}(\rho)=\sum_{n=1}^{\infty} b_{n} y_{n}^{\prime} \psi_{n}(\rho) \sin \left(h_{n}^{\prime} l\right) &
\end{array}
$$

The coefficients in equations (B.2.5) and (B.2.6) are determined in the usual manner by using the orthogonality properties of $\phi_{0}(\rho), \phi_{n}(\rho)$ and $\psi_{n}(\rho)$, and are

$$
\begin{array}{ll}
a_{0}=\frac{1}{k^{2}(1+R)} \int E_{t} \phi_{0} d A & \\
a_{n}=-\frac{1}{h_{n}^{2}} \int E_{t} \phi_{n} d A & n=1,2, \ldots \\
b_{n}=\frac{1}{h_{n}^{\prime}} \int E_{t} \psi_{n} \sin \left(h_{n}^{\prime} l\right) d A &
\end{array}
$$

where the integration in equation (B.2.7) is over the coaxial and cylindrical waveguide cross section. Substituting equation (B.2.7) into equation (B.2.6) gives

$$
\begin{array}{r}
k^{-2 \frac{1-R}{1+R}} y_{0} \phi_{0}(\rho) \int E_{t} \phi_{0}(\rho) d A+\sum_{n=1}^{\infty} \frac{y_{n}}{h_{n}^{2}} \phi_{n}(\rho) \int E_{t} \phi_{n} d A \\
=\sum_{n=1}^{\infty} \frac{y_{n}^{\prime}}{h_{n}^{\prime 2}} \psi_{n}(\rho) \sin \left(h_{n}^{\prime} l\right) \int E_{t} \psi_{n} d A
\end{array}
$$


The integration in $\rho$ is over $[a, b]$.

\section{B.3 Constructing the variational expression}

Multiply equation (B.2.8) by $E_{t}$ and integrate over $[a, b]$ to obtain

$$
\begin{aligned}
k^{-2} y_{i n} y_{0}\left[\int E_{t} \phi_{0}(\rho) d A\right]^{2} & =-\sum_{n=1}^{\infty} \frac{y_{n}}{h_{n}^{2}}\left[\int E_{t} \phi_{n}(\rho) d A\right]^{2} \\
& +\sum_{n=1}^{\infty} \frac{y_{n}^{\prime}}{h_{n}^{\prime}{ }^{2}} \sin \left(h_{n}^{\prime} l\right)\left[\int E_{t} \psi_{n}(\rho) d A\right]^{2},
\end{aligned}
$$

which is stationary with respect to a first-order variation in $E$, i.e., if $E$ is an arbitrary field in the region of the discontinuity, then $\delta y_{i n} / \delta E=0$ if $E=E_{t}$.

\section{B.4 Solution}

Let the electric field be expanded at $z=0$ as follows.

$$
E_{t}=C_{0} \phi_{0}(\rho)+\sum_{n=1}^{N} C_{n} \phi_{n}(\rho)
$$

Then

$k^{-2} y_{i n} y_{0}=\frac{\sum_{n=1}^{\infty} \frac{y_{n}^{\prime}}{h_{n}^{\prime}{ }^{2}} \sin \left(h_{n}^{\prime} l\right)\left[\int\left(C_{0} \phi_{0}+\sum_{n=t}^{N} C_{n} \phi_{n}\right) \psi_{n} d A\right]^{2}-\sum_{n=1}^{\infty} \frac{y_{n}}{h_{n}^{2}}\left[\int C_{n} \phi_{n}^{2} d A\right]^{2}}{\left[C_{0} 2 \pi A^{2} \ln \left(\frac{a}{b}\right)\right]^{2}}$ 
$=\left\{C_{0} \sum_{n=1}^{\infty} \frac{y_{n}^{\prime}}{h_{n}^{\prime 2}} \sin \left(h_{n}^{\prime} l\right)\left[\int \phi_{0} \psi_{n} d A\right\}^{2}+2 C_{0} \sum_{m=1}^{N} C_{m} \sum_{n=1}^{\infty} \frac{y_{n}^{\prime}}{h_{n}^{\prime}{ }^{2}} \sin \left(h_{n}^{\prime} l\right)\left[\int \phi_{0} \psi_{n} d A \cdot \int \phi_{n} \psi_{n} d A\right]\right.$

$+\sum_{r, s=1}^{N} C_{r} C \cdot \sum_{n=1}^{\infty} \frac{y_{n}^{\prime}}{h_{n}^{\prime}} \sin \left(h_{n}^{\prime} l\right)\left[\int \phi_{r} \psi_{n} d A \cdot \int \phi_{s} \psi_{n} d A\right]-\sum_{n=1}^{\infty} \frac{y_{n}}{h_{n}^{2}}\left[\int C_{n} \phi_{n}^{2} d A f^{2}\right]$

$/\left[C_{0} 2 \pi A^{2} \ln \left(\frac{a}{b}\right)\right]^{2}$

Setting $Y=k^{-2} y_{i n} y_{0}$, the stationary property of equation (B.4.2) implies

$\frac{\partial Y}{\partial C_{i}}=0 \quad i=0,1,2, \ldots, N$

The variation with respect to $C_{0}$ gives

$$
\begin{aligned}
\mathrm{C}_{0}\left(2 \pi A^{2} \ln \left(\frac{a}{b}\right)\right]^{2} Y= & C_{0} \sum_{n=1}^{\infty} \frac{y_{n}^{\prime}}{h_{n}^{\prime}{ }^{2}} \sin \left(h_{n}^{\prime} l\right)\left[\int \phi_{0} \psi_{n} d A\right]^{2} \\
& +\sum_{m=1}^{N} C_{m} \sum_{n=1}^{\infty} \frac{y_{n}^{\prime}}{h_{n}^{\prime 2}} \sin \left(h_{n}^{\prime} l\right)\left[\int \phi_{0} \psi_{n} d A \cdot \int \phi_{m} \psi_{n} d A\right]
\end{aligned}
$$

The variation with respect to $C_{k}$ gives

$$
\begin{aligned}
0=C_{k} \frac{y_{k}}{h_{k}^{2}}\left[\int \phi_{0} \psi_{n} d A f^{2}+C_{0} \sum_{n=1}^{\infty} \frac{y_{n}^{\prime}}{h_{n}^{\prime}{ }^{2}} \sin \left(h_{n}^{\prime} l\right)\left[\int \phi_{0} \psi_{n} d A \cdot \int \phi_{k} \psi_{n} d A\right]\right. \\
+\sum_{m=1}^{N} C_{m} \sum_{n=1}^{\infty} \frac{y_{n}^{\prime}}{h_{n}^{\prime 2}} \sin \left(h_{n}^{\prime} l\right)\left[\int \phi_{m} \psi_{n} d A \cdot \int \phi_{k} \psi_{n} d A\right]
\end{aligned}
$$

Solving equations (B.4.4) and (B.4.5) enables one to obtain 


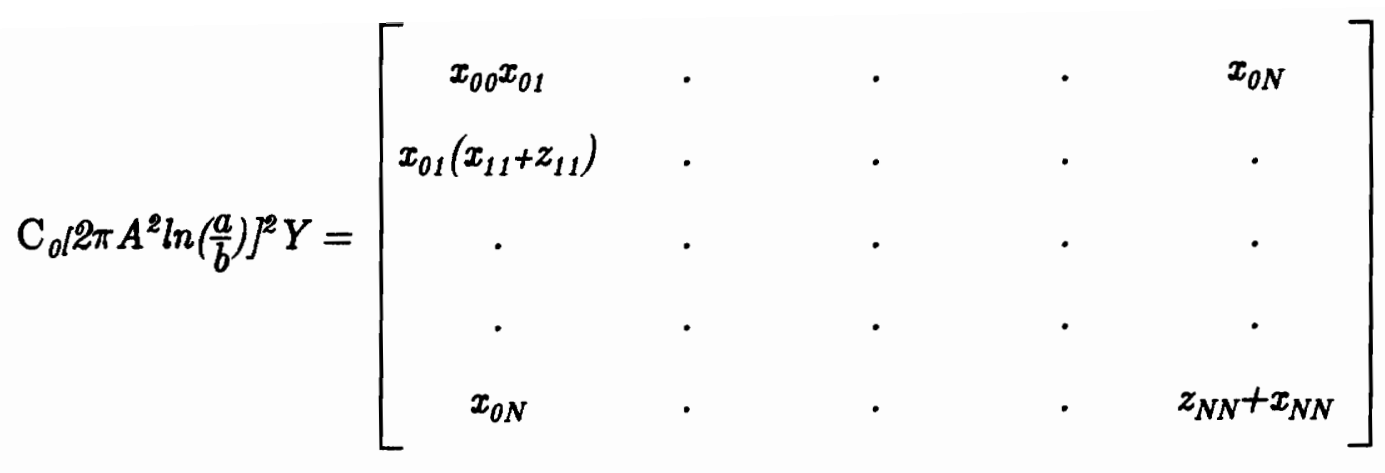

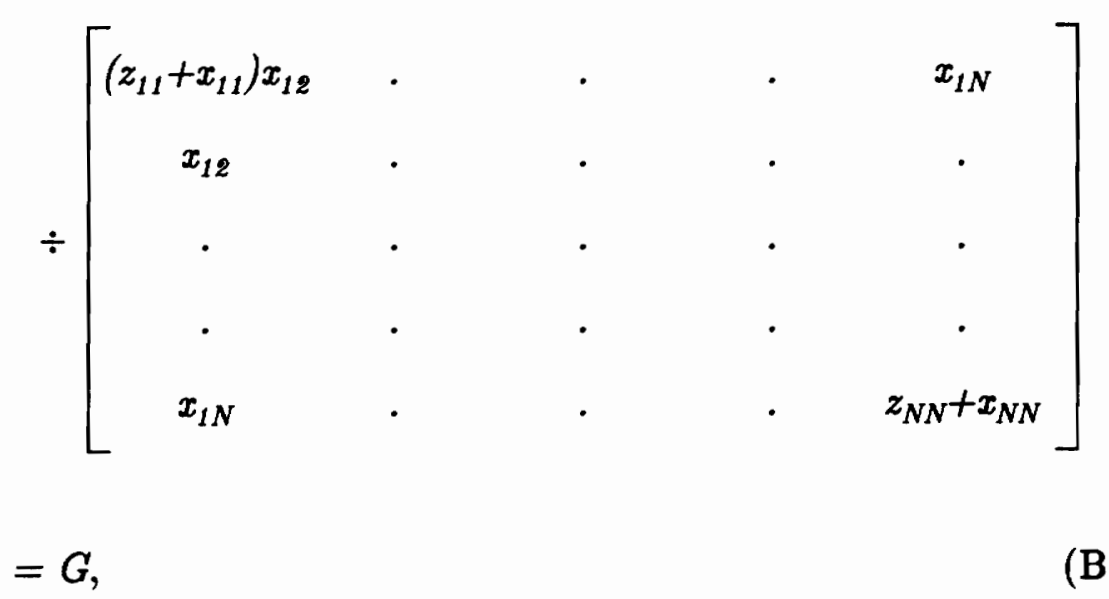

where the elements in equation (B.4.6) are

$x_{00}=\sum_{n=1}^{\infty} \frac{y_{n}^{\prime}}{h_{n}^{\prime 2}} \sin \left(h_{n}^{\prime} l\right)\left[\int \phi_{0} \psi_{n} d A\right]^{2}$

$x_{0 m}=\sum_{n=1}^{\infty} \frac{y_{n}^{\prime}}{h_{n}^{\prime 2}} \sin \left(h_{n}^{\prime} l\right)\left[\int \phi_{0} \psi_{n} d A \cdot \int \phi_{m} \psi_{n} d A\right]$

$x_{m k}=\sum_{n=1}^{\infty} \frac{y_{n}}{h_{n}^{\prime}{ }^{2}} \sin \left(h_{n}^{\prime} l\right)\left[\int \phi_{m} \psi_{n} d A \cdot \int \phi_{k} \psi_{n} d A\right]$

$z_{k k}=\frac{y_{k}}{h_{k}^{2}}\left[\int \phi_{0} \psi_{n} d A\right]^{2}$ 
Finally, the capacitance is

$$
C=\frac{y_{\text {in } y_{0}}}{\omega}=\frac{k^{2} G}{\omega\left[2 \pi A^{2} \ln \left(\frac{a}{b}\right]^{2}\right.}
$$




\section{BIBLIOGRAPHY}

[1] Andrews, J. R., "Automatic network measurements in the time domain," Proc. IEEE, 66, 1978, pp. 414-423.

[2] Miller, E. K., ed. Time-Domain Measurements in Electromagnetics, Van Nostrand Reinhold Company Inc., New York, pp. 95-121, 1986.

[3] Nicolson, A. M., "Broad-band microwave transmission characteristics from a single measurement of the transient response, ${ }^{n}$ IEEE Trans. Instrum. Meas., IM-17, 1968, pp. 395-402.

[4] Grove, W. M., "Sampling for oscilloscopes and other RF systems: DC through X-band," IEEE Trans. Microwave Theory Tech., MTT-14, 1966, pp. 629635 .

[5] Shannon, C. E., "A mathematical theory of communication," Bell Syst. Tech. J., 27, 1948, pp.623-656.

[6] Samulon, H. A., "Spectrum analysis of transient response curves," Proc. IRE, Feb., 1951, pp. 175-186. 
[7] Cooley, J. W. and Tukey, J. W., "An algorithm for the machine calculation of complex Fourier series," Math. Comp., 19, 1965, pp. 297.

[8] Stuckert, P., "Computer augmented oscilloscope system," IEEE Trans. Instrum. Meas., IM-18, 1969, pp. 299-306.

[9] Norris, N. and Hanst, R., "Picosecond beam monitors and data acquisition system,” IEEE Trans. Nucl. Sci., NS-16, 1969, pp. 927-931.

[10] Gans, W. L. and Andrews, J. R., "Time Domain Automatic Network Analyzer for Measurement of RF and Microwave Components," NBS TN-672, National Bureau of Standards, Boulder, Colorado, 1975.

[11] Gans, W., "Calibration and error analysis of a picosecond pulse waveform measurement system at NBS,” Proc. IEEE, 74, 1986, pp. 86-90.

[12] Riad, S. M., "Modeling of the HP-1430A feedthrough wideband (28 ps) sampling head," IEEE Trans. Instrum. Meas., IM-31, 1982, pp. 110-115.

[13] HP 54120 user documentation (installation/operating manual), HewlettPackard company, 1987.

[14] Berlin, H. M., and Getz, F. C., Jr., Principles of Electronic Instrumentation and Measurement, Merill Publishing Company, Columbus, Ohio, 1988. 
[15] Gordiol, F. E., Lossy Transmission Line, Artech House, 1987.

[16] Somlo, P. I., "The computation of coaxial line step capacitances," IEEE Trans. Microwave Theory Tech., MTT-15, 1966, pp. 48-53.

[17] HP associates, "Network analysis: specifying calibration standards for the HP-8510 network analyzer," Product Note: 8510-5A, Hewlett-Packard Company, 1988.

[18] Volk, W., Applied Statistics for Engineers, McGraw-Hill Book Company, New York, 1958.

[19] Beauchamp, K. G., Signal Processing, Gorge Allen \& Unwin Ltd., London, 1973.

[20] Papoulis, A., Signal Analysis, McGraw-Hill Book Company, New York, 1977

[21] Gupta, K. C., Garg, R. and Chadha, R., Computer-aided Design of Microwave Circuits, Artech House, 1981.

[22] Nahman, N. S., and Guillaume, M. E., "Deconvolution of time domain waveforms in the presence of noise," NBS TN-1047, National Bureau of Standards, Boulder, Colorado, 1981. 
[23] Parruck, B., and Riad, S. M., "An optimization criterion for iterative deconvolution," IEEE Trans. Instrum. Meas., IM-32, 1983, pp. 137-140.

[24] Andrews, J. R., "10 ps risetime transmission \& TDR measurements using the PSPL 4015B 15 ps pulse generator \& HP 54124A $50 \mathrm{GHz}$ oscilloscope,” Application Note: AN-5a, Picosecond Pulse Labs., July, 1992.

[25] Shaarawi, A. M., and Riad, S. M., "Computing the complete-FFT of a steplike waveform," IEEE Trans. Instrum. Meas., IM-35, 1986, pp. 91-92.

[26] Marple, S. L., Jr., Digital Spectral Analysis, Prentice-Hall, Inc., Englewood Cliffs, 1987.

[27] Harrington, R. F., Time-Harmonic Electromagnetic Fields, McGraw-Hill Book Company, New York, 1961.

References of general interest on the subject

[1] Andrews, J. R., and Arthur, M. G., "Spectrum amplitude -- definition, generation and measurements," NBS TN-699, National Bureau of Standards, Boulder, Colorado, 1977.

[2] Nahman, N. S., "Picosecond-domain waveform measurements," Proc. IEEE, 66,1978, pp. 441-454. 
[3] Wigington, R. L., and Nahman, N. S., "Transient analysis of coaxial cables considering skin effect," Proc. IRE, 45, 1957, pp. 166-174.

[4] Nahman, N. S., and Jickling, R. M., "Frequency domain measurement of baseband instrumentation," NBSIR 73-330, National Bureau of Standards, Boulder, Colorado, 1973.

[5] Arthur, M. G., "Impulse spectral intensity -- What is it?," NBSIR 74-365, National Bureau of Standards, Boulder, Colorado, 1974.

[6] Gans, W. L., and Nahman, N. S., "Fast Fourier transform implementation for the calculation of network frequency domain transfer functions from time domain waveforms," NBSIR 73-303, National Bureau of Standards, Boulder, Colorado, 1973.

[7] Bracewell, R. N., The Fourier Transform and Its Applications, McGraw-Hill Book Company, New York, 1978.

[8] Papoulis, A., The Fourier Integral and Its Applications, McGraw-Hill Book Company, New York, 1962.

[9] Su, K. L., Time-Domain Synthesis of Linear Networks, Prentice-Hall Inc, New Jersey, 1971. 
[10] Nelson, R. E., and Coryell, M. R., "Electrical parameters of precision, coaxial, air-dielectric transmission lines," NBS Monograph 96, National Bureau of Standards, Boulder, Colorado, 1966.

[11] Bailey, A. E., ed., Microwave Measurement, Peter Peregrinus Ltd., London, 1985.

[12] Choma, J., Electrical Networks, John Wiley \& Sons, Inc., New York, 1985.

113] Riad, S. M., and Nahman, N. S., "Applications of the homomorphic transformation to time domain measurement problems," NBSIR 78-881, National Bureau of Standards, Boulder, Colorado, 1978.

[14] Nahman, N. S., Andrews, J. R., Gans, W. L., Guillaume, M. E. Lawton, R. A., Ondrejeka, A. R., and Young, M., "Applications of time-domain methods to microwave measurements," IEE Proc., 127, 1980, pp. 99-106. 


\section{CURRICULUM VITAE}

Mr. Wansheng Su was born in China in 1953. He received his B.S. degree from the Northwest Telecommunication and Engineering Institute, Xi'an, China, in 1975, and M.S. degree from the University of Science and Technology of China, Beijing, China, in 1981. Since 1981, he has worked at the National Institute of Metrology (NIM), China. In 1989, he was on a leave from NIM as a visiting scholar with the Electrical Engineering Department, Virginia Polytechnic Institute and State University. He is a member of the IEEE Instrumentation and Measurement Society.

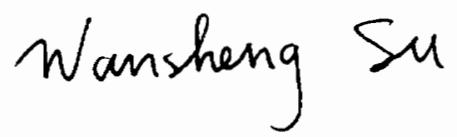

